Florida International University FIU Digital Commons

5-30-1991

\title{
Design and analysis of a folded meander dipole antenna using the MININEC program
}

Pedro A. Barros

Florida International University

DOI: $10.25148 /$ etd.FI14050449

Follow this and additional works at: https://digitalcommons.fiu.edu/etd

\section{Recommended Citation}

Barros, Pedro A., "Design and analysis of a folded meander dipole antenna using the MININEC program" (1991). FIU Electronic Theses and Dissertations. 1428.

https://digitalcommons.fiu.edu/etd/1428 


\section{DESIGN AND ANALYSIS OF \\ A FOLDED MEANDER DIPOLE ANTENNA \\ USING THE MININEC PROGRAM}

BY

PEDRO A. BARROS 


\section{ABSTRACT OF THE THESIS}

\section{DESIGN AND ANALYSIS OF A FOLDED MEANDER DIPOLE ANTENNA, USING THE MININEC PROGRAM. \\ by}

\section{Pedro A. Barros}

Florida International University, 1991

Miami, Florida

Professor Tadeusz Babij, Major Professor

The Minl-Numerlcal Electromagnet1c Code (MININEC) program, a PC-Compatible version of the powerful NEC program, is used to design a new type of reduced-size antenna. The validity of the program to model simple well-known antennas, such as dipoles and monopoles, is first shown. More complex geometries such as folded dipoles, and meander dipole antennas are also analysed using the program. The final design geometry of a meander folded dipole is characterized with MININEC, yielding results that serve as the basis for the practical construction of the antenna. Finally, the laboratory work with a prototype antenna is described, and practical results are presented. 


\title{
FLORIDA INTERNATIONAL UNIVERSITY \\ Mlami, Florida
}

\section{DEBIGN OF AND ANALYBIS OF A FOLDED MEANDER DIPOLE ANTENNA USING THE MININEC PROGRAM}

\begin{abstract}
A Thesis submitted in partial satisfaction of the the requirements for the degree of Master of Science In Electrical Englneering
\end{abstract}

by

Pedro A. Barros 
To Professors: Tadeusz Bablf, Wanaba subbarao, and Mark Hagmann.

Th1s thes1s, having been approved in respect to form and mechanlcal execution 13 referred to you for judgement upon its substantial merit.

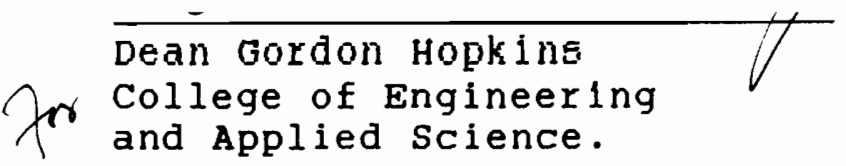

The Thesls of Pedro A. Barros is, approved.
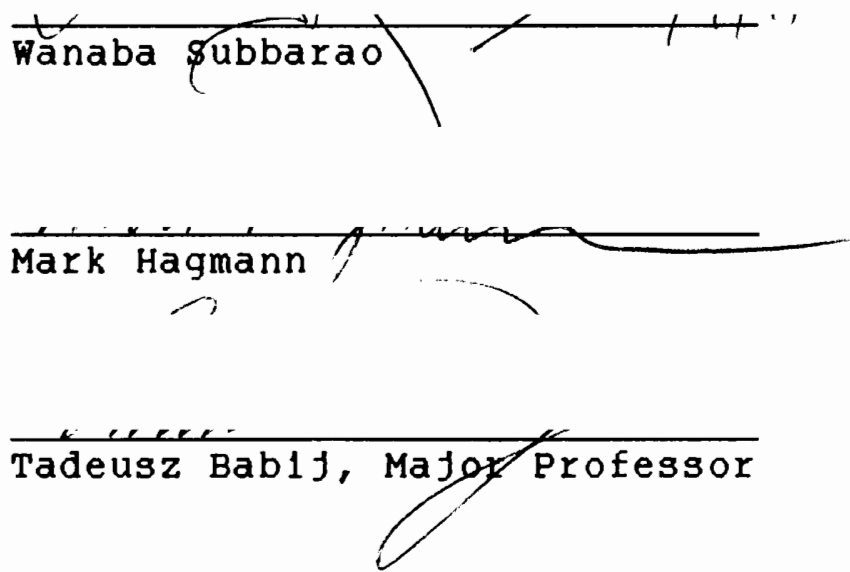

Date of Examination: 5-30.

$$
1 \text {. }
$$

Dean Richard Campbell

Division of Graduate studies 


\section{ACKNOWLEDGEMENTS :}

I wish to express my gratitude to Dr. Tadeusz Babif, my thesis advisor, for his guidance and assistance throughout the study and development of this thesis.

I also wish to thank Dr. Wanaba Subbarao, and Dr. Mark Hagmann for their interest in my work and their help in reviewing the manuscript, and to all the professors whose lectures made completing this thesis, an easier task.

Finally, my deep gratitude goes to my wife, Marisa, for her support and encouragement throughout the school years. 


\section{CONTENTS}

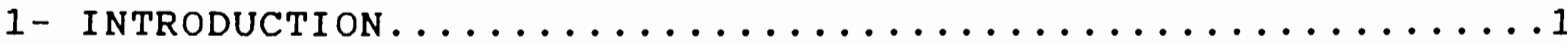

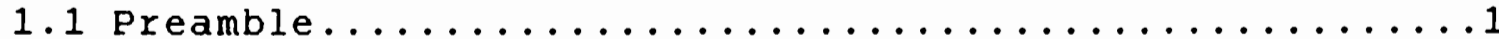

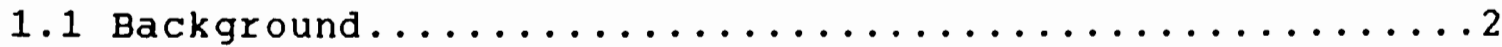

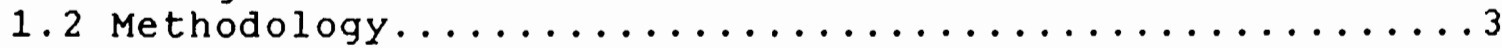

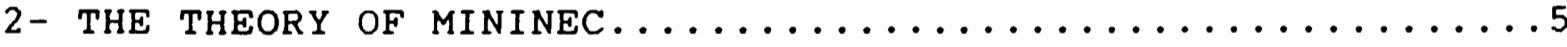

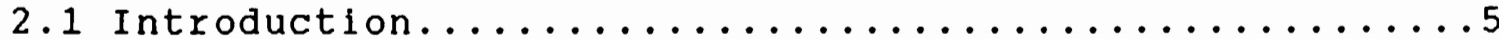

2.2 The Electric Field Integral and its Solution.........5

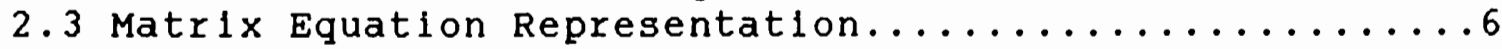

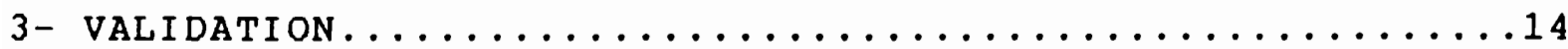

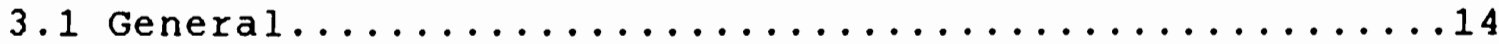

3.2 Quarter Wave Monopole on Conducting Ground..........14

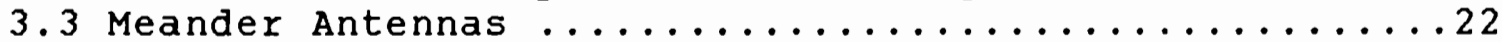

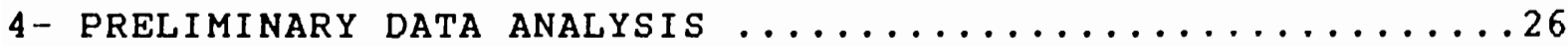

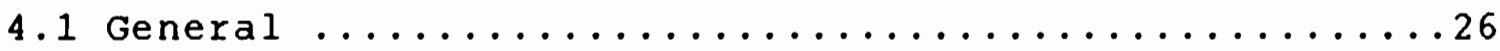

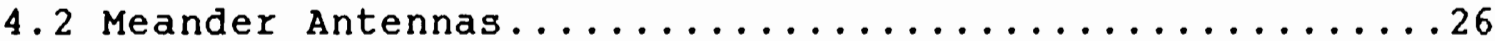

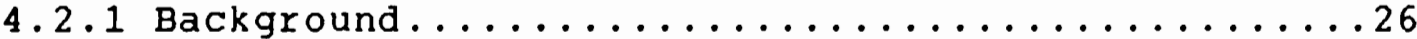

4.2.2 Effect of $w$ on Antenna Characteristics.........27

4.2 .3 Effect of $N$ in Antenna Characteristics.........32

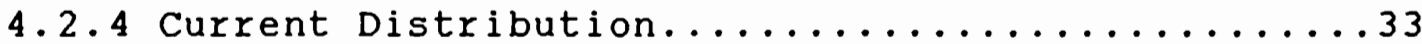

4.3 Cylindrical Antennas and the Dipole.............36

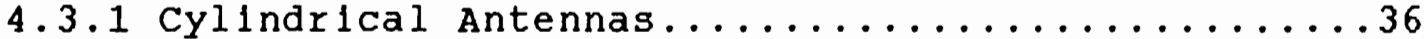

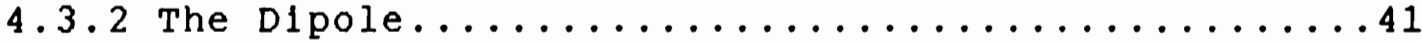

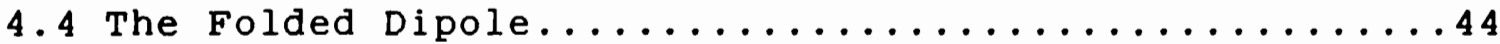

4.4 .1 Theoretical Analysis....................44

4.4.2 Impedance Transformation as a Function of

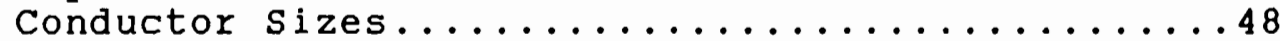

4.4.3 Modelling a Folded Dipole with MININEC........51

4.4.4 step-up Transformation Ratio.............58

4.4.5 Folded Dipole Impedance..............665

4.4.6 Modelling First Resonance with MININEC.......668

4.4 Conclusions........................ 72 
5- THE MEANDER FOLdEd DIPOLE $\ldots \ldots \ldots \ldots \ldots \ldots \ldots \ldots \ldots \ldots \ldots$

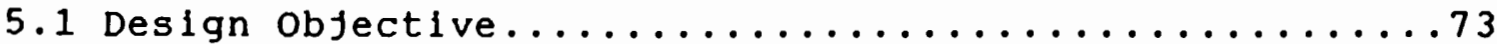

5.2 Analysis of the Meander Dipole...............75

5.3 Considerations on Segmentation Density..........84

5.4 Analysis of the Meander Folded Dipole............86 5.4.1 Effect of wire Separation in Antenna

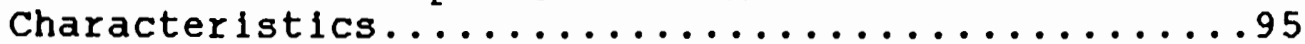

5.4.2 Folded Meander Dipole Radiation Pattern........97

5.4.3 Impedance Transformation Ratio..............99

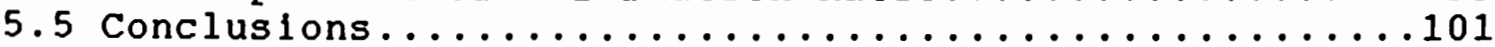

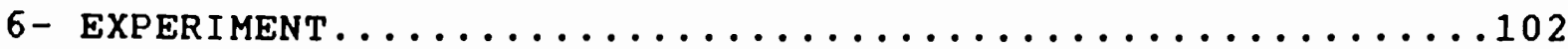

6.1 Measurement Techniques and Equipment...........102

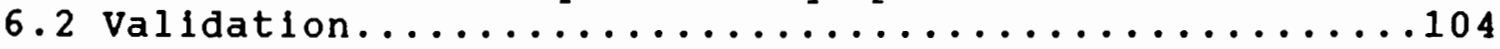

6.2 .1 Monopole over Conducting Ground............104

6.2 .2 Considerations on Experimental Results........106

6.3 Test of a Folded Meander Dipole.................110

6.3 .2 Impedance Measurement.....................113

6.3 .3 Bandwidth of the Folded Meander Dipole.........117

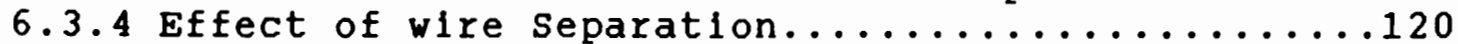

6.3 .5 Impedance Transformation Ratio............121

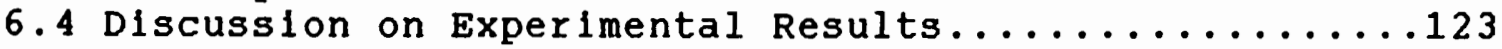

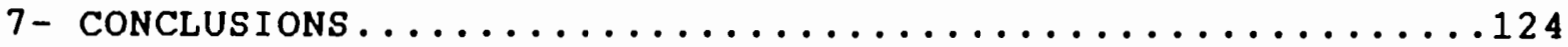

7.1 Basic Theory of Meander Antennas..............124

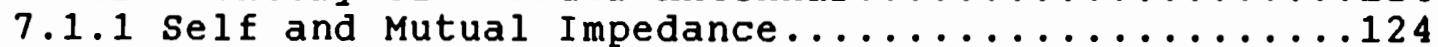

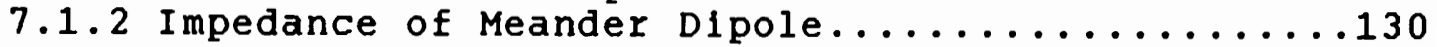

7.1.3 Radiated Field of a meander Dipole..........132

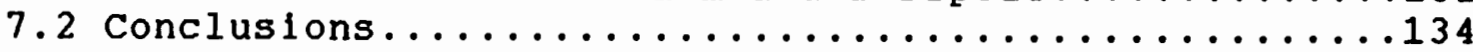

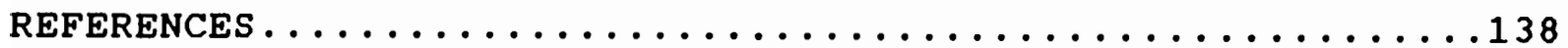


CHAPTER 2:

2.1: Geometry of an arbitrarily oriented wire............12

2.2: Wire segmentation with pulses for current and charges....13

CHAPTER 3 :

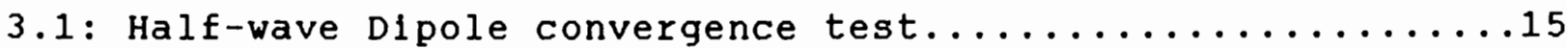

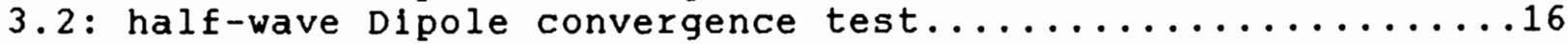

3.3: Input Resistance of a quarter-wave monopole...........18

3.4: Input Reactance of a quarter-wave monopole............19

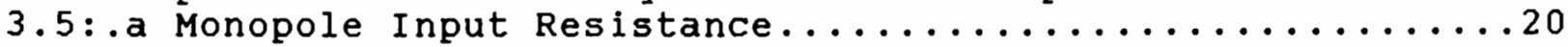

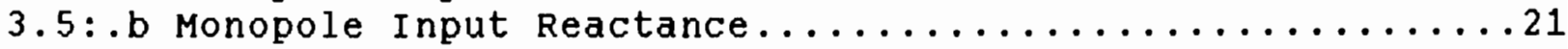

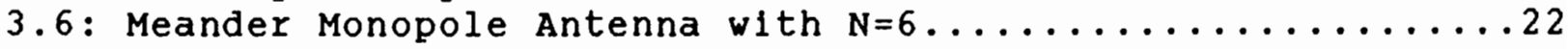

\section{CHAPTER 4:}

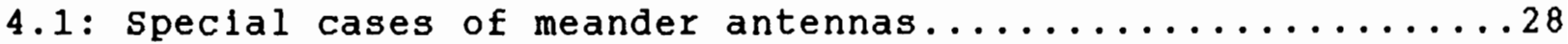

4.2: Impedance Curve for Meander Monopole with $w=0.25$

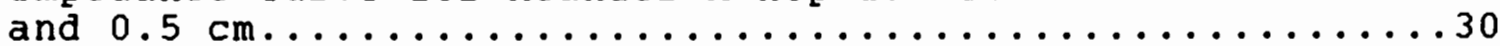

4.3: impedance of a conventional Monopole of length $=50 \mathrm{~cm} \ldots 31$

4.4.a: Current Distribution (Magnitude): Conventional and

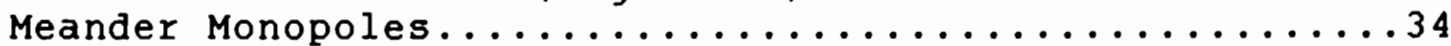

4.4.b: Current Distribution (Phase): Conventional and Meander

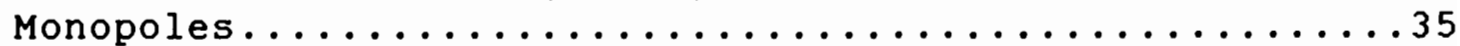

4.5.a: Input Resistance of Monopole over Conducting Ground, for different Diameter to Length Ratios, Versus Electrical

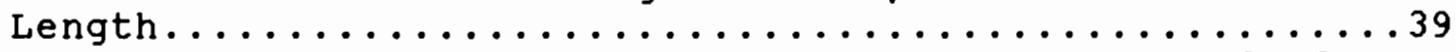

4.5.b: Input Reactance of Monopole over Conducting Ground, for different Diameter to Length Ratios, Versus Electrical

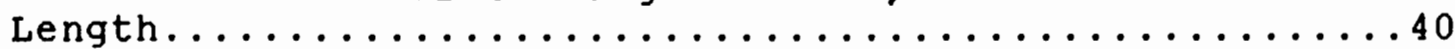

4.6: Resonant Resistance of Half-Wave Dipole and Length Factor versus Half-Wavelength to wire-Dlameter Ratio.........42

4.7: VSWR Versus Frequency ona 72 ohm Transmission Line feeding two Dipoles having two values of wire Radius..........43

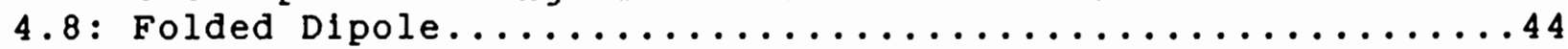

4.9: Equivalent Representation of the Folded Dipole........45

4.10: Equivalent Radius of a Rectangle as a Function of the

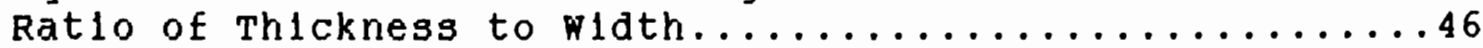

4.11: Equivalent Circuit of a Folded Dipole..............48

4.12: Diameter Ratio Versus Impedance Transformation Rat10...49

4.13: Impedance Transformation Ratio Versus Diameter Ratio....50

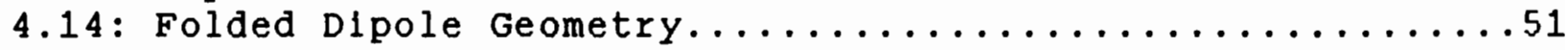

4.15: Impedance of a Folded Dipole with $d=1.0 \mathrm{~cm} \ldots \ldots \ldots 5$

4.16: Impedance of a Folded Dipole with d=6.0 cm..........56

4.17: Vertical Radiation Pattern of a Folded Dipole.........59

4.18: Extrapolated Impedance Transformation Rat1o..........62

4.19: Impedance Transformation Ratio Versus Radius step

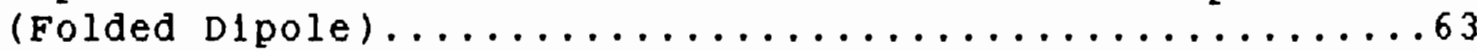


4.20: Impedance of a Folded D1pole...................65

4.21: Input Impedance of a Folded dipole as a Function of its

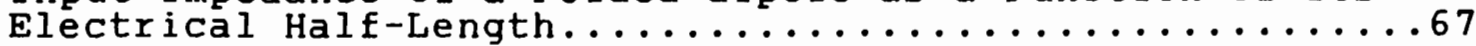

4.22: Input Resistance of a Folded Dipole................70

4.23 Input Reactance of a Folded Dipole...............71

CHAPTER 5 :

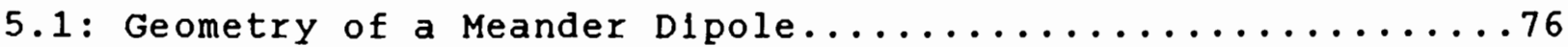

5.2: Meander Dipole Impedance Chart. $(w=2.5 \mathrm{~cm}.) \ldots \ldots \ldots . .60$

5.3: Meander Dipole Impedance Chart. $(w=3.0 \mathrm{~cm}.) \ldots \ldots \ldots . . .81$

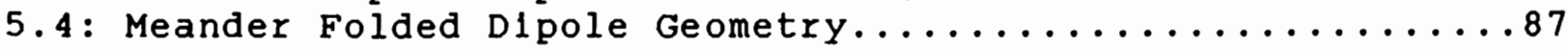

5.5: Folded Meander Dipole Input Resistance..............90

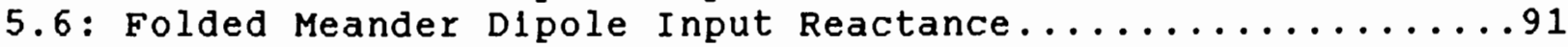

5.7: Folded Meander D1pole Impedance Chart...............94

5.8: Folded Meander Dipole vertical Radiation Pattern........98

CHAPTER 6 :

6.1: Test set-up for Monopole over Ground Plane............105

6.2: Measured Resistance of a Wire Monopole..............108

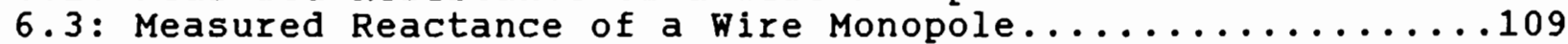

6.4: Geometry of Folded Meander Dipole with Image

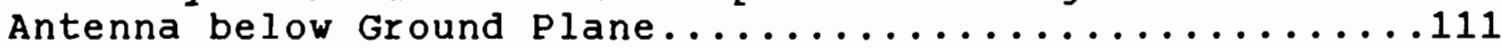

6.5: Test Set-up for a Folded Meander Monopole............113

6.6: Measured Resistance of a Folded Meander Monopole........116

6.7: Measured Reactance of a Folded Meander Monopole........117

6.8: Impedance of a Folded Meander Dipole....................

CHAPTER 7:

7.1: Dipole Positioning for Mutual Coupling.............127

7.2: Mutual Impedance of side-by-side Arrangement

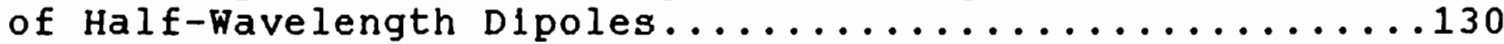

7.3: Equivalent Representation of a Meander Dipole...........132

7.4: Two Isotropic Sources Separated by a Distance r........134 


\section{LIST OF TABLES}

CHAPTER 3.0

3.1 Segmentation Density Conversion...............17

3.2 Meander Monopole Impedance vs. Number

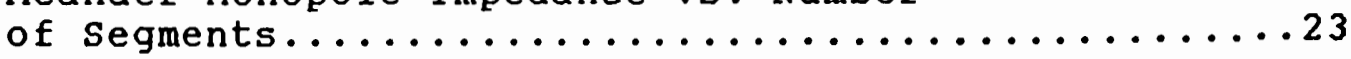

CHAPTER 4.0

4.1 Effect of Wire separation on Characteristics of

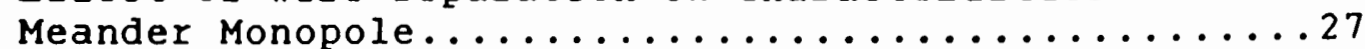

4.2 Bandwidth of Meander Monopole with $\mathrm{N}=2 \ldots \ldots \ldots \ldots \ldots 29$

4.3 Effect of $N$ on Meander Antenna Characteristics......32

4.4 Functions $R(k I)$ and $X(k l)$ contained in the Formula of a Center Driven Dipole................

4.5 Effect of wire Separation on the Resonant Resistance of a Folded Dipole................52

4.6 Bandwidth of a Folded Dipole as wire separation

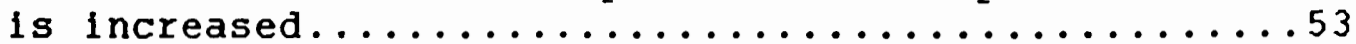

4.7 Impedance of a Folded Dipole with $\mathrm{d}=1.0 \mathrm{~cm} \ldots \ldots . . .54$

4.8 Impedance of a Folded Dipole with $\mathrm{d}=6.0 \mathrm{~cm} . \ldots \ldots \ldots 57$

4.9 Impedance Transformation Ratio of a

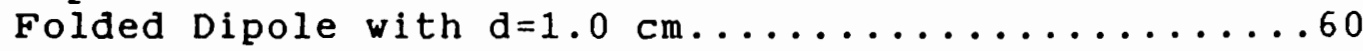

4.10 Impedance Transformation Ratio of a Folded Dipole with $d=6.0 \mathrm{~cm} \ldots \ldots \ldots \ldots \ldots 1$ 4.11 First Reasonance of a Folded Dipole............669

CHAPTER 5.0

5.1 Resonant Resistance of a Meander Dipole.........77

5.2 Effect of $w$ on Meander Dipole Bandwidth.........78

5.3 Meander Dipole $(w=2.5 \mathrm{~cm}$.$) Impedance............79$

5.4 Meander Dipole $(w=3.0 \mathrm{~cm}$.$) Impedance............82$

5.5 Folded Meander Dipole Coordinates..............88

5.6 Folded Meander Dipole Input Impedance............92

5.7 Folded Meander Dípole Impedance around

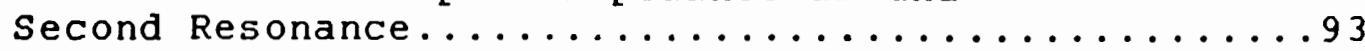

5.8 Effect of $w$ in Antenna Resonant Resistance........95

5.9 Meander Folded Dipole Bandw1dth..............96

5.10 Impedance Transformation Ratio as a Function of Conductor Diameter Ratio................99

CHAPTER 6.0

6.1 Measured Impedance of a Thin Wire Monopole........107

6.2 Measured Impedance of Folded Meander Dipole........115 
CHAPTER 6.0 (Continued)

6.3 Measured Impedance of Meander Folded Monopole

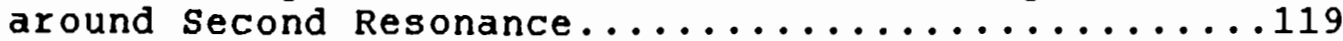

6.4 Effect of Wire Separation on Experimental

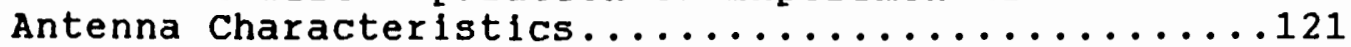

6.5 Effect of Changes in Conductor Diameter on

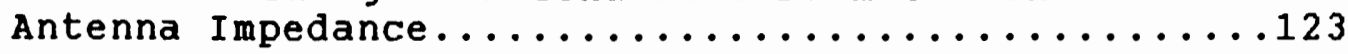




\subsection{INTRODUCTION}

\subsection{PREAMBLE:}

This Thesis describes the design of a new type of antenna, using one of the most promising CAD tools for antenna design existing today: the MININEC program.

MININEC is a shortened version of the powerful Numerical Electromagnetic Code (NEC) program for antenna analysis and design used mainly with mainframe computers. MININEC has the great advantage, for engineers and engineering students, that it can be run in any IBM PC-Compatible microcomputer, with 512 kbytes of memory. It represents a most useful tool for the analysis and design of thin-wire antennas of moderate complexity.

The antenna design presented here, stems from the author's special interest in electrically small and reduced-size antennas.

There are indeed many practical applications for reduced-size antennas, from aerospace to military, to portable (and small) communications equipment, to make research in this field not only interesting, but necessary.

One of the most interesting reduced-size antennas investigated in recent years are meander antennas (3). These antennas are extensively described in the next chapters and analysed with MININEC. They provide a good reduction in size, and maintain most of the important radiation characteristics of conventional antennas. Furthermore, they are basically wire-antennas, which makes them very suitable for analysis with MININEC.

Before any practical design could be accomplished, it was necessary to start with applying the program to some simple and well known antennas such as monopoles and dipoles, and then to proceed with the analysis of meander monopoles and dipoles. The purpose of this preliminary effort, that covers a good part of this paper, was threefold.

First, it was necessary to validate the results of MININEC, by comparing them with known reference data. Validation tests were accomplished on monopoles and dipole antennas, for this reason.

Second, it was important to gain enough experience with the operation of the program. Convergence tests were on different antenna geometries, to test the accuracy of the results and the inherent limitations of the program.

Third, since meander antennas are obviously the cornerstone of our design, it was important to run a complete analysis of these geometries, and of the folded dipole. 
Before the digital computer, the design of antennas was more a cut-and-try operation than an accurate mathematical process. [1] For complex-geometry antennas, this operation involved many handcalculations, iterations, simplification of antenna geometry and, of course, long hours of practical laboratory work.

Along came the computer, and scientists and engineers rushed to convert proven algorlthms into computer programs or to develop new algorithms suitable for use in computers as analysis or design tools.

Today, the most powerful of these computer tools available for the design and analysis of linear antennas is the Numerical Electromagnetic code (NEC). It is a highly user-oriented computer code offering a comprehensive capability for analysis of the interaction of electromagnetic waves with conducting structures. The program is based on the numerical solution of integral equations for the currents induced on the structure by an exciting field. [11]

NEC combines an integral equation fot smooth surfaces with with one for wires to provide convenient and accurate modeling for a wide range of applications.

A NEC model model may 1nclude nonradiating networks and transmission lines, perfect and imperfect conductors, lumped element loading, and ground planes. The ground planes may be perfectly or imperfectly conducting. Excitation may be via an applied voltage source or incident plane wave.

The output may include induced currents and charges, near or far zone electric or magnetic fields, and impedance or admittance. Many other commonly used parameters such as gain and directivity, power budget, and antenna to antenna coupling are also available. [11]

NEC was developed at the Lawrence Livermore National Laboratory under the joint sponsorship of the U.S. Navy and Air Force. The NEC user community comprises 17 government facilities, 11 domestic universities, over 20 domestic companies and at least 8 foreign organizations. [1] [11]

NEC is a poweful tool for many engineering applications. It is ldeal for modeling co-site antenna environments in which the interaction between antenna and environment cannot be ignored.

However, in many applications, the extensive full capability of NEC is not really required ( for example, in cases where the antenna and its environment are not very complex, or the information sought requires only a simplified model.)

In addition, NEC require the use of a a large main frame computer. These computer systems are expensive and not readily available. 
Even when such computer 1 avallable, heavy demand may result in slow turn-around, even for relatively simple NEC runs.

The obvious solution to this problems was the development of a "stripped-down" version of NEC that retains only the the basic solution and the most frequently-used options and that can be implemented in a simple micro-computer system. This program is written in Basic and has approplately been named MININEC.

MININEC offers many of the required NEC options but makes use of the Basic language that is compatible with most microcomputers. MININEC is only suitable for for small problems on small computers compatible with the IBM format. However, with the use of a compiler the program can be used to analyse antenna problems of practical sizes.

\section{METHODOLOGY}

The performance of an antenna system is generally evaluated in terms of the following parameters: [2]

\section{PARAMETERS}

Impedance

Mlsmatch or VSWR

Bandwidth.

Power (voltage) limit.

Coupling

Near Electric Field

Near Magnetic Field

Far Field Patterns

Gain

Beam-width
PERFORMANCE MEASURE

Compatibility to RF equipment

(Notably transmitters and recelvers) 
Scale model measurement (cont'd.)

Computational extrapolation from empirical data.

Exact EM analysis

Approximate EM solutions
Depending on antenna, it may require a large investment in resources to establish and maintain. Some phenomena do not scale linearly. Interference can also be a problem.

This approach suffers from the same problems of any measurement scheme with the added necessity to maintain and retrleve from potentially large data bases.

Exact analysis is limited to rather simple canonical geometries for which an exact solution may be possible. This amounts to a very small set of possible geometries.

Requires a thorough understanding of the constraints to avoid inappropriate use and invalid data. Solutions are approximate and must be interpreted in a way consistent with the constraints of the model. Usually this technique requires access to computer resources.

Some of the approximate methods of solution include finite difference techniques, quasi-optical techniques such as the geometric theory of diffraction, and method of moments technique. [2]

MININEC makes use of the technique known as the method of moments, to calculate the performance parameters of a thin wire antennas, following these steps:

1.- Formulate the thin wire model in terms of an appropriate set of integral-differential equations.

2.- Reduce the integral-differential equations to asystem of linear algebraic equations. This is accomplished in the method of moments by making certain simplifying assumptions, including the selection of a set of current expansion and testing functions. These assumptions will be treated in a later chapter.

3.- specify the environment. That 1s, include the effects of a ground plane or free space, locate and account for all excitation sources and incorporate all loads into the solution.

4.- Solve the system of linear equations for the currents.

5.- Use the currents to compute the antenna performance parameters. 


\subsection{INTRODUCTION}

The MININEC program is based on the numerical solution of an integral equation representation of the electric fields. There is nothing new or unusual about this formulation. The real advantage is that the solution technique results in a relatively compact computer code. The discussion that follows is transcribed from [1].

It is included in this paper because it is considered important in understanding the validity of the program as an antenna design tool.

\subsection{THE ELECTRIC FIELD INTEGRAL EQUATION AND ITS SOLUTION}

In solving wire antenna problems, it has become customary to make several assumptions that are valid for thin wires. They are:

1- That the wire radius is very small with respect to the length.

2- The wire radius is very small with respect to the wavelength.

Because it is necessary to subdivide wires into short segments, the radius is assumed small with respect to segment length as well, so that the currents can be assumed to be axially directed ( there are no azimuthal current components).

Figure 2.1 gives the geometry of a typical arbitrarily oriented wire. For simplicity, it is assumed that the wire is straight, but the theory applies equally to bent configurations. Also shown is the wire broken into segments or subsections.

The vector and scalar potentials are given by: [2]

$$
\begin{aligned}
& \pi=\frac{\mu}{4 \pi} \int_{s} I(s) s(s) k(r) d s \\
& \Phi=\frac{1}{4 \pi_{t}} \int_{s} q(s) k(r) d s
\end{aligned}
$$

Where the integration is along the length $s$ of the wire and:

$$
k(r)=\frac{1}{2 \pi} \int_{-\pi}^{\pi} \frac{e^{-j k r}}{r} d \phi
$$

where $r$ is the distance from the source point of the current to the observation point of the field, and the integration is over the angular variation around the wire.

The linear charge density (via the continulty equation) is: 


$$
q(s)=-\frac{1}{j \omega} \frac{d l}{d s}
$$

The method of moments solution used in the MININEC system is based on relating the current distribution of the wire to the incldent field on the surface of the wire. The integral equation relating the incident field E-inc. and the vector and scalar potentials is:

$$
-E_{\text {inc }} \cdot \bar{\xi}=-j \omega \pi \cdot \xi-\xi \cdot \nabla \phi
$$

\section{?. 3 MATRIX EQUATION REPRESENTATION}

Equation (1.4) is solved in MININEC by using the following procedure. The wires are divided into equal segments and, as shown in figure 2.1, the vectors $r_{n}, n=0,1,2, \ldots N$ are defined with respect to the global coordinate origin, 0 . The unit vector parallel to the wire axis for each segment shown are defined as:

$$
\bar{s}_{n+1 / 2}=\frac{\bar{r}_{n+1}-\bar{r}_{n}}{\left|\bar{r}_{n+1}-\bar{r}_{n}\right|}
$$

Pulse testing and pulse expansion functions used in MININEC are defined as follows:

$$
\begin{gathered}
P_{n}(s)=1 \text {, for } s_{n-1 / 2}<s<s_{n+1 / 2} \\
P_{n}(s)=0 ; \text {, otherwise }
\end{gathered}
$$

Where the points $s n+1 / 2$ and $s n-1 / 2$ designate segment midpoints

$$
\begin{aligned}
& s_{n+1 / 2}=\frac{s_{n+1}+s_{n}}{2} \\
& s_{n-1 / 2}=\frac{s_{n}+s_{n-1}}{2}
\end{aligned}
$$


A difference approximation 13 applied to equation(1.3) to compute the charge. Thus, as shown in figure 2.2 , the charge can be represented as pulses displaced from the current by a half pulse width.

substituting(1.11) into (1.10) produces a system of equations that can be expressed in matrix form. Each matrix element $\mathrm{Zm}, \mathrm{n}$ associated with the nth. current pulse and the sm observation point on the wire surface involves scalar and vector potentials terms with integrals of the form:

$$
\psi_{m, u, v}=\int_{0.0}^{0 \cdot} k\left(s_{m}-s^{\prime}\right) d s
$$

where

$$
k\left(s_{m}-s^{\prime}\right)-\frac{1}{2 \pi} \int_{-\pi}^{\pi} \frac{e^{-j k r_{m}}}{r_{m}} d \phi
$$

and

$$
r_{m}-\left[\left(s_{m}-s^{\prime}\right)^{2}+4 a^{2} \sin ^{2}\left(\frac{\phi}{2}\right)\right]^{1 / 2}
$$

Equation(1.12) does not lend 1tself to stralghtforward integration because of the singularity at $r=0$. The $1 / r$ can be substracted from the integrand and then added as a separate term, to yield:

$$
k\left(s_{m}-s^{*}\right)=\frac{1}{2 \pi} \int_{-\pi}^{\pi} \frac{d \phi}{r_{m}}+\frac{1}{2 \pi} \int_{-\pi}^{\pi} \frac{e^{-j k r}-1}{r_{m}} d \phi
$$

The flrst term of (1.15) can be rewritten as an elliptic integral of the first kind:

$$
\frac{\beta}{\pi a} F\left(\frac{\pi}{2}, \beta\right)-\frac{1}{2 \pi} \int_{-\pi}^{\pi} \frac{d \phi}{r_{m}}
$$

where:

$$
\beta=\frac{2 a}{\left[\left(s_{m}-s^{-}\right)^{2}+4 a^{2}\right]^{1 / 2}}
$$


The following approximation can be used:

$$
F\left(\frac{\pi}{2}, \beta\right)=\left[\sum_{i=0}^{4} a_{i} m^{\prime}\right]+\left[\sum_{i=0}^{4} b_{i} m^{\prime}\right] \ln (1 / m)
$$

where :

$$
m=1-\beta^{2}=\frac{\left(s_{m}-s^{\prime}\right)^{2}}{\left(s_{m}-s^{\prime}\right)^{2}+4 a^{2}}
$$

and

$$
\begin{array}{ll}
a_{0}=1.38629436112 & b_{0}=.5 \\
a_{1}=.09666344259 & b_{1}=.1249859397 \\
a_{2}=.03590092383 & b_{2}=.06880248576 \\
a_{3}=.03742563713 & b_{3}=.03328355346 \\
a_{4}=.01451196212 & b_{4}=.00441787012
\end{array}
$$

Thus as sm approaches s'

$$
\frac{\beta}{\pi a} F\left(\frac{\pi}{2}, \beta\right) \Rightarrow-\frac{1}{\pi a} \ln \left[\frac{\left|s_{m}-s^{\prime}\right|}{8 a}\right]
$$

and this singularity is also substracted from k(sm-s') Thus

$$
\begin{gathered}
k\left(s_{m}-s^{\prime}\right)--\frac{1}{\pi a} \ln \left[\frac{\left|s_{m}-s^{\prime}\right|}{8 a}\right] \\
+\frac{\beta F\left(\frac{\pi}{2}, \beta\right)+\ln \left[\frac{\left|\theta_{m}-\dot{\theta}^{\prime}\right|}{\theta 0}\right]}{\pi a}+\frac{1}{2 \pi} \int_{-\alpha}^{\pi} \frac{e^{-\pi / r_{m}-1}}{r_{m}} d \phi
\end{gathered}
$$

This equation is substituted into equation(1.12) and written as

$$
\int_{1 .}^{1 .} k\left(s_{m}-s^{\prime}\right) d s=I_{1}+I_{2}+I_{3}
$$


or in terms of the global coordinates,

$$
\begin{aligned}
& \bar{r}_{n+1 / 2}=\frac{\bar{r}_{n+1}+\bar{r}_{n}}{2} \\
& \bar{r}_{n-1 / 2}=\frac{\bar{r}_{n}+\bar{r}_{n-1}}{2}
\end{aligned}
$$

It is assumed that the components of the vectors $-E$ inc and $A$ in equation (4) are sufficiently smooth over each segment that their respective values on each segment may be replaced. by those taken at the point sm on the wire. The pulse functions of (1.6) are then used as testing functions on (1.4) resulting in:

$$
\begin{gathered}
E_{\text {inc }}\left(s_{m}\right) \cdot\left[\left(\frac{s_{m}-s_{m-1}}{2}\right) s_{m-1 / 2}+\left(\frac{s_{m+1}-s_{m}}{2}\right) s_{m \cdot 1 / 2}\right]- \\
j \omega \pi\left(s_{m}\right) \cdot\left[\left(\frac{s_{m}-s_{m-1}}{2}\right) s_{m-1 / 2}+\left(\frac{s_{m+1}-s_{m}}{2}\right) s_{m+1 / 2}\right] \\
+\phi\left(s_{m+1 / 2}\right)-\phi\left(s_{m-1 / 2}\right)
\end{gathered}
$$

The vector quantities in brackets are simply:

$$
\left(\bar{r}_{m+1 / 2}-\bar{r}_{m-1 / 2}\right)
$$

so that (9) can be written as:

$$
\begin{aligned}
E_{\text {inc }}\left(s_{m}\right) \cdot\left(\bar{r}_{m+1 / 2}-\bar{r}_{m-1 / 2}\right) & =j \omega \pi\left(s_{m}\right) \cdot\left(\bar{r}_{m+1 / 2}-\bar{r}_{m-1 / 2}\right) \\
& +\phi\left(s_{m+1 / 2}\right)-\phi\left(s_{m-1 / 2}\right)
\end{aligned}
$$

The currents are expanded in pulses centered at the functions of adjacent segments as lilustrated in flgure 2.1. Note that pulses are omitted from the wire ends. This is equivalent to placing a half pulse of zero amplitude at each end. This imposes a boundary condition for a zero current at unattached wire ends. The current expansion can be written as:

$$
l(s)-\sum_{n=1}^{N} I_{n} P_{n}(s)
$$


$I_{1}, I_{2}$ and $I_{3}$ are defined as:

$$
I_{1}=-\frac{1}{\pi a} \int_{1 .}^{1 \cdot} \ln \left[\frac{\left|s_{m}-s^{\cdot}\right|}{8 a}\right]^{-} d s^{\prime}=\left.\frac{8}{\pi} u(1-\ln |u|)\right|_{u_{1}} ^{u_{2}}
$$

where

$$
u_{1}=\frac{s_{u}-s_{m}}{8 a}
$$

and

$$
u_{2}=\frac{s_{v}-s_{m}}{8 a}
$$

similarly,

$$
I_{2}=\int_{1 .}^{80} \frac{\beta F\left(\frac{\pi}{2}, \beta\right)+\ln \left[\frac{\left|\theta_{m}-\theta^{\prime}\right|}{8 a}\right]}{\pi a} d s
$$

This integral has a well behaved integrand and can be integrated numericaliy. The integration is broken up into two integrals over the ranges $(\mathrm{Su}, \mathrm{Sm})$ and $(\mathrm{Sm}, \mathrm{Sv})$ for best accuracy. Gaussian quadrature is used for the numerical integration. The number of points in the integration is automatically selected by consideration of the source to observation distance. The final integral is:

$$
I_{3}=\frac{1}{2 \pi} \int_{0 .}^{0 \cdot} \int_{-\pi}^{\pi} \frac{e^{-j k r_{m}}-1}{r_{m}} d \phi
$$

This integrand is nonsingular and can also be integrated numerically, To obviate the need for double integration, it is convenient to approximate the integral by replacing $r_{m}$ of equation(1.14) by a reduced kernel approximation. Thus,

$$
I_{3}=\int_{0}^{e_{0}} \frac{e^{-i k r \cdot}-1}{r_{0}} d s
$$

where,

$$
r_{a}=\sqrt{\left(s_{m}-s^{\prime}\right)+a^{2}}
$$

This integral can be integrated numerically by the same procedure as for $I_{2}$. 
Thus, equation(1.12) with 1 ts singularity problem is evaluated by adding equation $I_{1}$ of equation(1.21), $I_{2}$ of equation(1.22), and $I_{3}$ of equation (1.24)

By substitution, the matrix equation to be solved is:

$$
\left[V_{m}\right]-\left[Z_{m n}\right]\left[I_{n}\right]
$$

where

$$
\begin{aligned}
& Z_{m n}=-\frac{1}{4 \pi j \omega \epsilon}\left[k^{2}\left(\bar{r}_{m+1 / 2}-\bar{r}_{m-1 / 2}\right) \cdot\left(\xi_{n+1 / 2} \psi_{m, n, n+1 / 2}+\xi_{n-1 / 2} \psi_{m, n-1 / 2, n}\right)\right. \\
&-\frac{\psi_{m+1 / 2, n, n+1}}{s_{n+1}-s_{n}}+\frac{\psi_{m+1 / 2, n-1, n}}{s_{n}-s_{n-1}} \\
&\left.+\frac{\psi_{m-1 / 2, n, n+1}}{s_{n+1}-s_{n}}-\frac{\psi_{m-1 / 2, n-1, n}}{s_{n}-s_{n-1}}\right]
\end{aligned}
$$

and

$$
V_{m}-E_{m c}\left(s_{m}\right) \cdot\left(\bar{r}_{m+1 / 2}-\bar{r}_{m-1 / 2}\right)
$$

[Zmn] is a square matrix and [In] and [Vm] are column matrices with $m=1,2,3 \ldots N$ and $n=1,2,3 \ldots N$ for $N$ total unknowns (i.e. $N$ is the total number of current pulses) The extension of these equations to two or more coupled wires follows the same line of development and is covered in reference 2 .

The column vector [Vm] represents an applied voltage that superimposes a tangencial electric field along the wire for a distance of one segment length centered at the location of the current pulses. Hence, for a transmitting antenna, all elements of [Vm] are set to zero except for the element(s) of the segment(s) located at the desired feed point(s). For an incldent plane wave, all elements of [Vm] must be assigned a value depending on the strength, polarization (or orientation) and angle of incidence of the plane wave. The applied voltage source (transmit case), however is the only ready-made or programmed option in the current calculation algorithm.

As stated before, the $[\mathrm{zm}, \mathrm{n}]$ matrix in equation(1.25) is filled by the evaluation of an elliptic integral and use of the Gaussian quadrature for numerical integration. The solution to(1.25) can be accomplished by using any one of a number of of standard matrix solution techniques The MININEC version used in the antenna design, described in this paper, uses a triangular decomposition (LU decomposition) with the Gaussian elimination procedure and partial pivoting. 


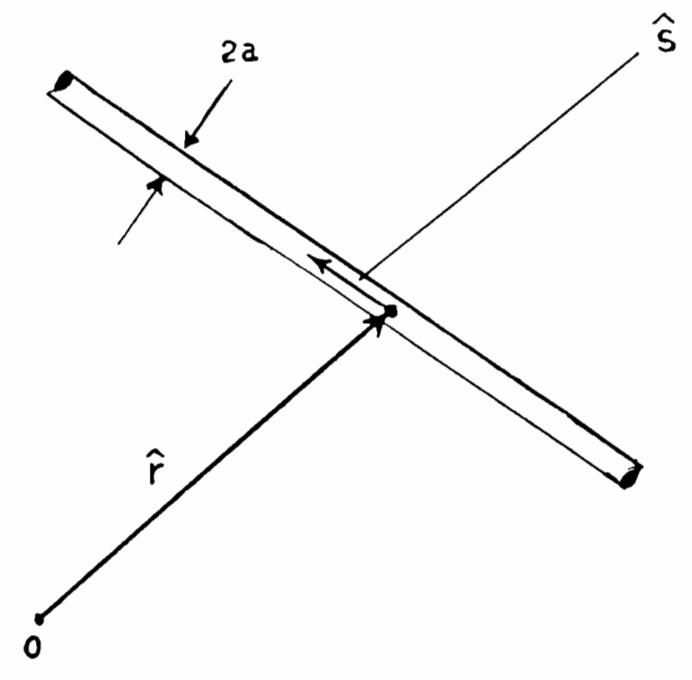

(a) ARBITRARILY ORIENTED WIRE.

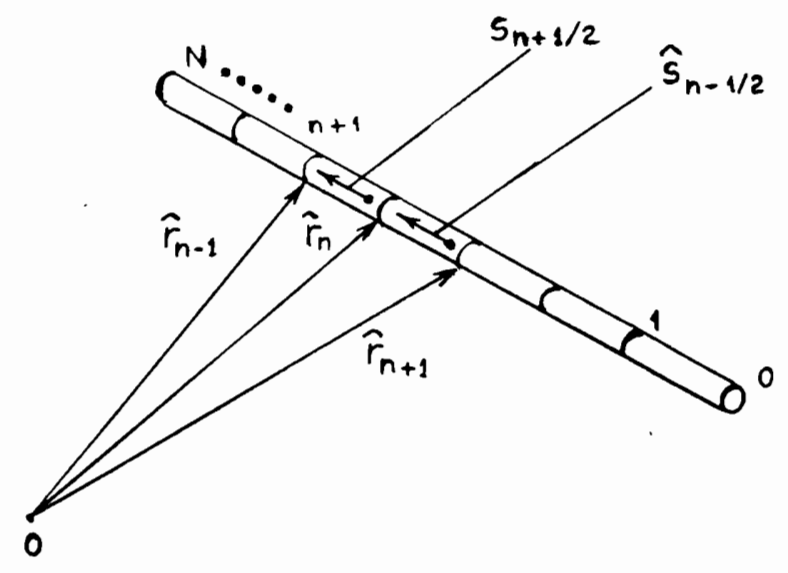

(b) SEGMENTATION SCHEME FOR THE SAME WIRE.

FIGURE 2.1: GEOMETRY OF AN ARBITRARILY ORIENTED WIRE ( FROM [1]) 


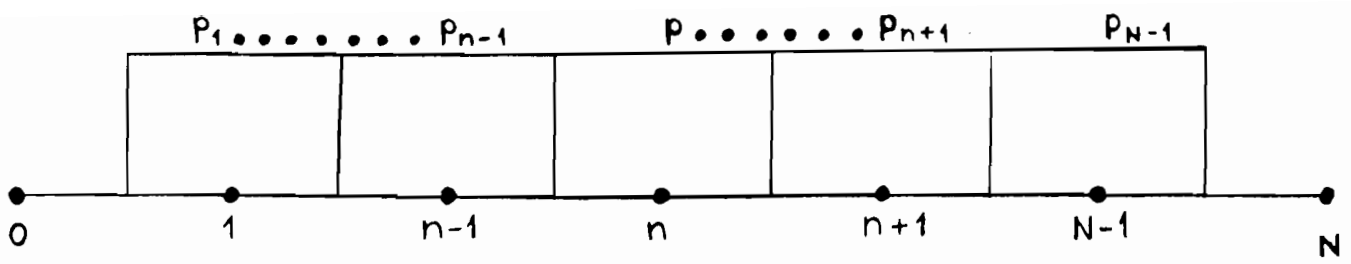

(a) UNWEIGHTED CURRENT PULSES

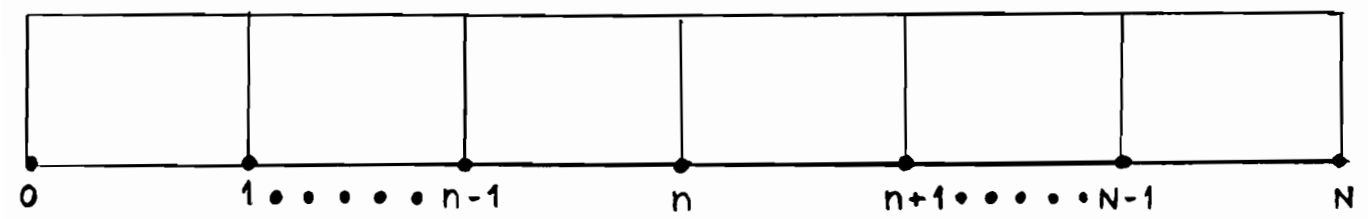

(b) UNWEIGHTED CHARGE REPRESENTATION.

FIGURE 2.2: WIRE SEGMENTATION WITH PULSES FOR CURRENT AND CHARGES ( FROM [1]) 


\subsection{VALIDATION}

\subsection{GENERAL}

Validation of the MININEC system is based on the comparison of the results of the program, when applied to a known geometry, with practical or theoretical data for that geometry.

There are extensive discussions in references 1 and 2 on this subfect. In particular, convergence tests are done for three cases: an electrically short dipole (much shorter than the flrst resonance length), a dipole near resonance, and a dipole near antiresonance.

The segmentation scheme for each speciflc geometry seems to be important in obtaining accurate results. In general, it can be sald that there is an optimum number of segments for each geometry. The exact number can only be determined on the basis of experlence in using the program. Flgures 3.1 and 3.2 show the convergence of MININEC data as the segment number is increased, on a center-fed half wave dipole in free space, when compared with at theoretical value published by $R$. W.P. KING. [5]

It can be seen that the optimum number of segments for this antenna would be around 32 segments. However, the program run time increases with the number of segments. (greater complexity of the solution). Probably, a smaller segment number would also be adequate, since the results do not differ more than $5 \%$ for a number greater than 8 segments. In fact, the reactive curve does not converge any further for more than 12 segments.

Another Important conclusion that can be drawn from these convergence tests is the fact that, in general, segment (or wire) radius is inversely proportional to the accuracy of the result. For small radil $(r \ll 0.01$ wire length) the accuracy of the result will not vary much, but it will tend to converge to the theoretical value. However, for design purposes, the radius of the wire should reflect the "true" radius utilized in the finished antenna. This will norma-lly be a one or two millimeters at the most.

\section{.2 QUARTER MAVE MONOPOLE ON CONDUCTING GROUND}

As a further validation test, the impedance of a quarter-wave monopole on conducting ground was calculated for several frequencles, corresponding to a length-to-wavelength ratlo from 0 to 0.75 (well past antiresonance). 


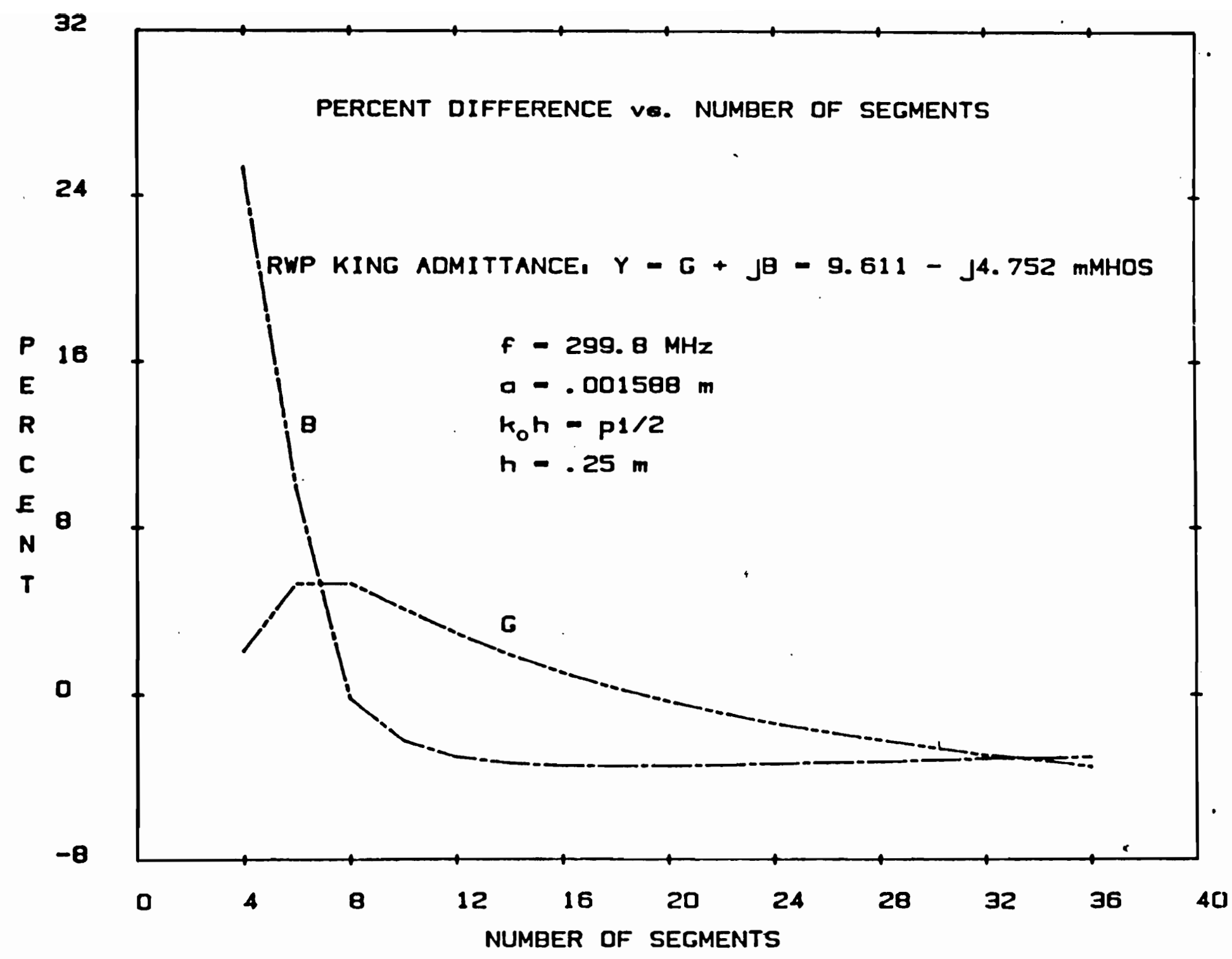

FIGURE 3.1: HALF-WAVE DIPOLE CONVERGENCE TEST (FROM [1]) 


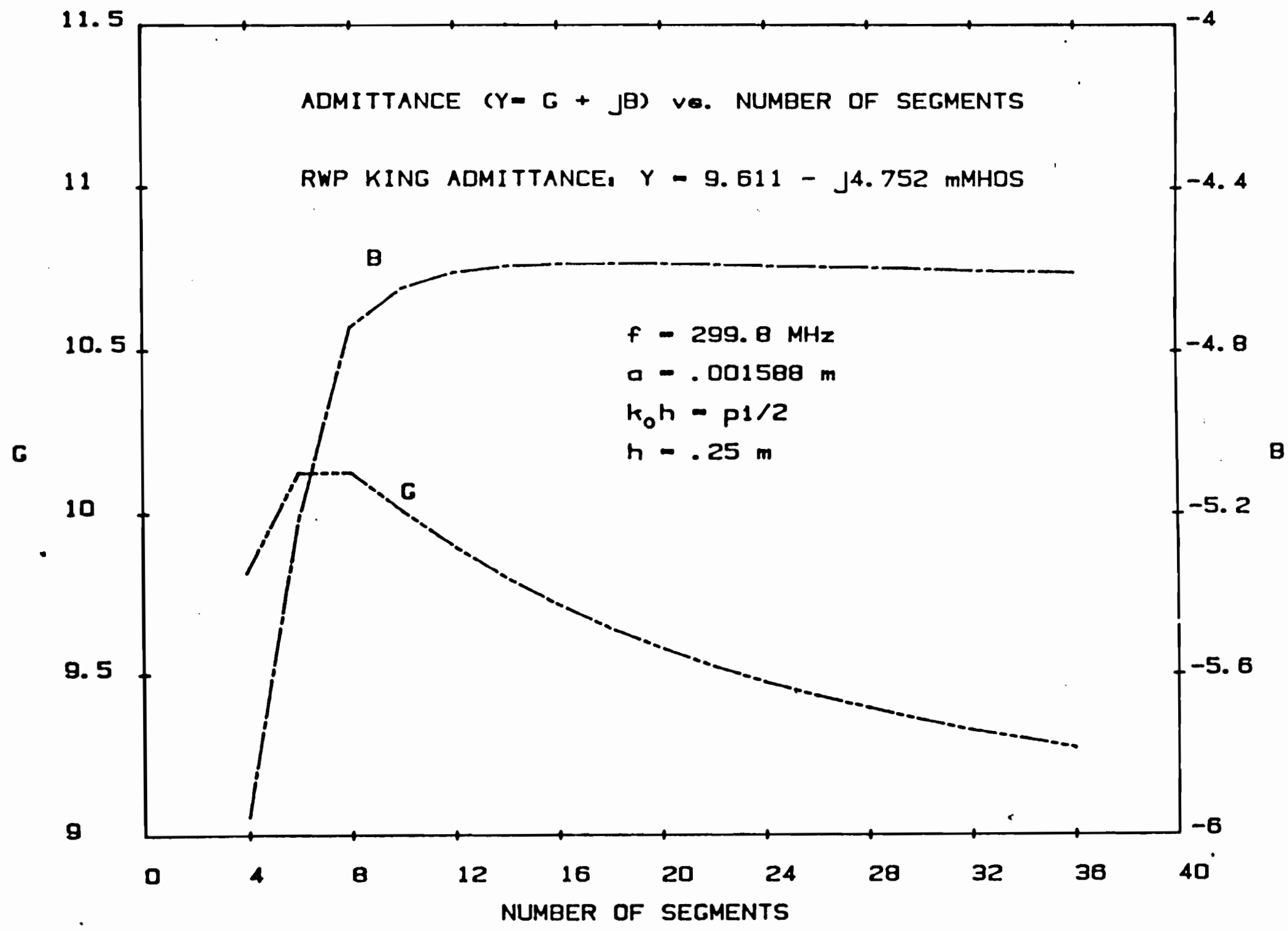

FIGURE 3.2: HALF-WAVE DIPOLE CONVERGENCE TEST (FROM [1]) 
The geometry was chosen to have a wire length of 100 times the wire radius, and having 16 segments. The antenna had a length of 0.25 mts. which means resonance occurs at $300 \mathrm{MHz}$.

The MININEC version used was that from [ 2 ] : The MININEC System by Artech House.

Both the Real and Imaginary part of the impedance were calculated and compared to theoretical values obtained from [4] (Kraus)

A Erequency sweep was accomplished to obtain the curve.

Figures 3.3 and 3.4 show the curves of real and imaginary parts of the quarter wavelength monopole on a perfectly conducting ground It can be seen that both curves show excellent coincidence with theoretical values for length/wavelength ratios close to 0.25 (resonance for a monopole). The values differ more for antiresonance but they do not exceed $10 \%$ of difference in the worst case.

The next step was to compute the input impedance of the described monopole for different segment densities. The segmentations scheme started as low as 2 segments for this single-wire antenna and was increased to a maximum of 50 segments.

The following table 3.1 shows the results of this test.

TABLE 3.1: SEGMENTATION DENSITY CONVERSION

SEGMENT NO.

2

4

10

20

30

40

50
INPUT IMPEDANCE (Ohms)

$$
\begin{aligned}
& 36.95+j 23.87 \\
& 39.95+j 19.40 \\
& 41.60+j 20.42 \\
& 42.3+j 21.63 \\
& 42.6+j 22.20 \\
& 42.8+j 22.53 \\
& 42.93+j 22.69
\end{aligned}
$$

This results were compared with the practical results from [5], obtained by R.W.P King, for a monopole with a wire radius-towavelength ratio of 0.001 and a length-to-wavelength ratio of 0.25 . This impedance is:

$$
z_{\text {ref }}=43.7+j 22.84
$$




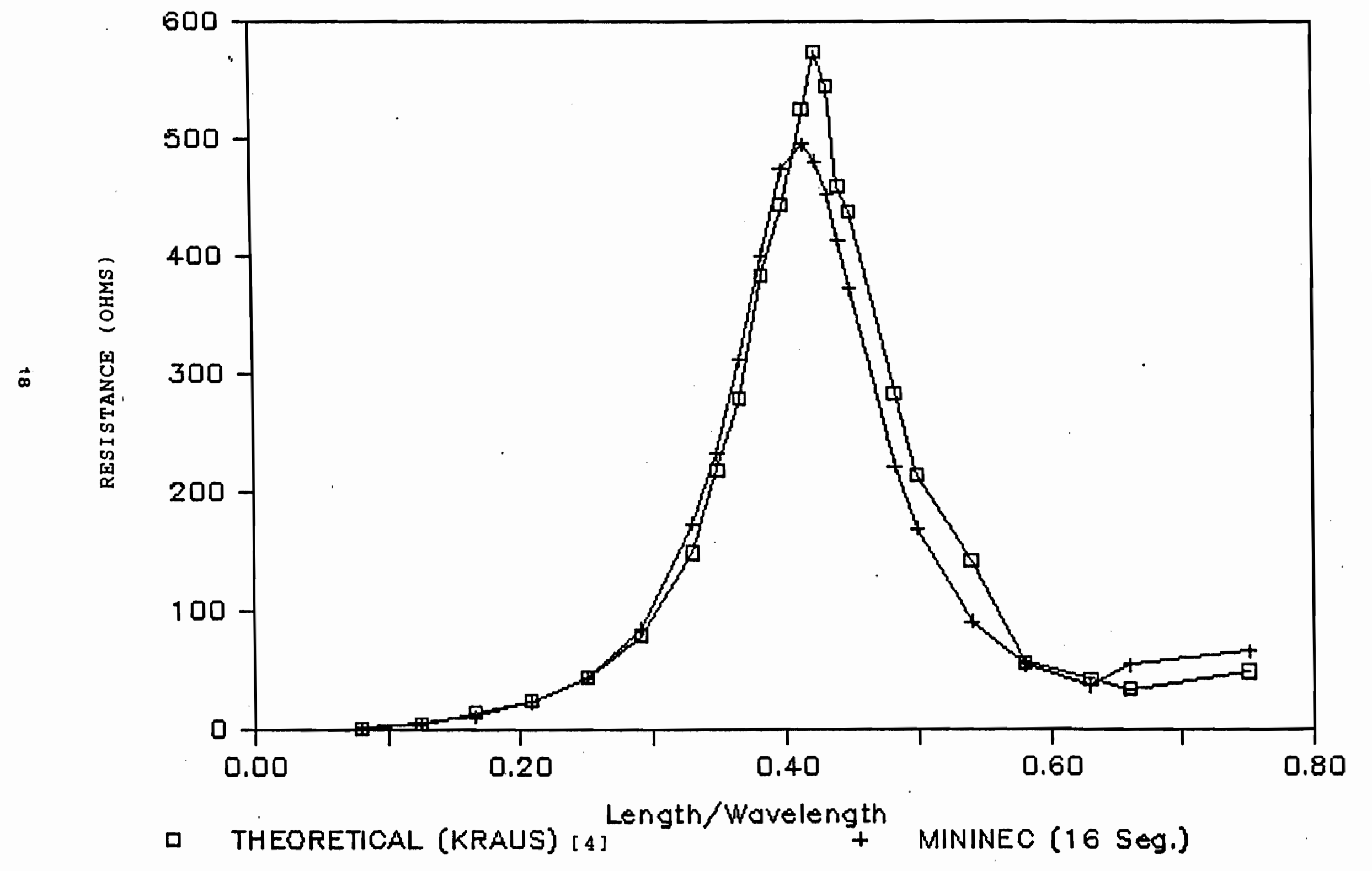


FIGURE 3.4: INPUT REACTANCE OF A QUARTER WAVELENGTH MONOPOLE.

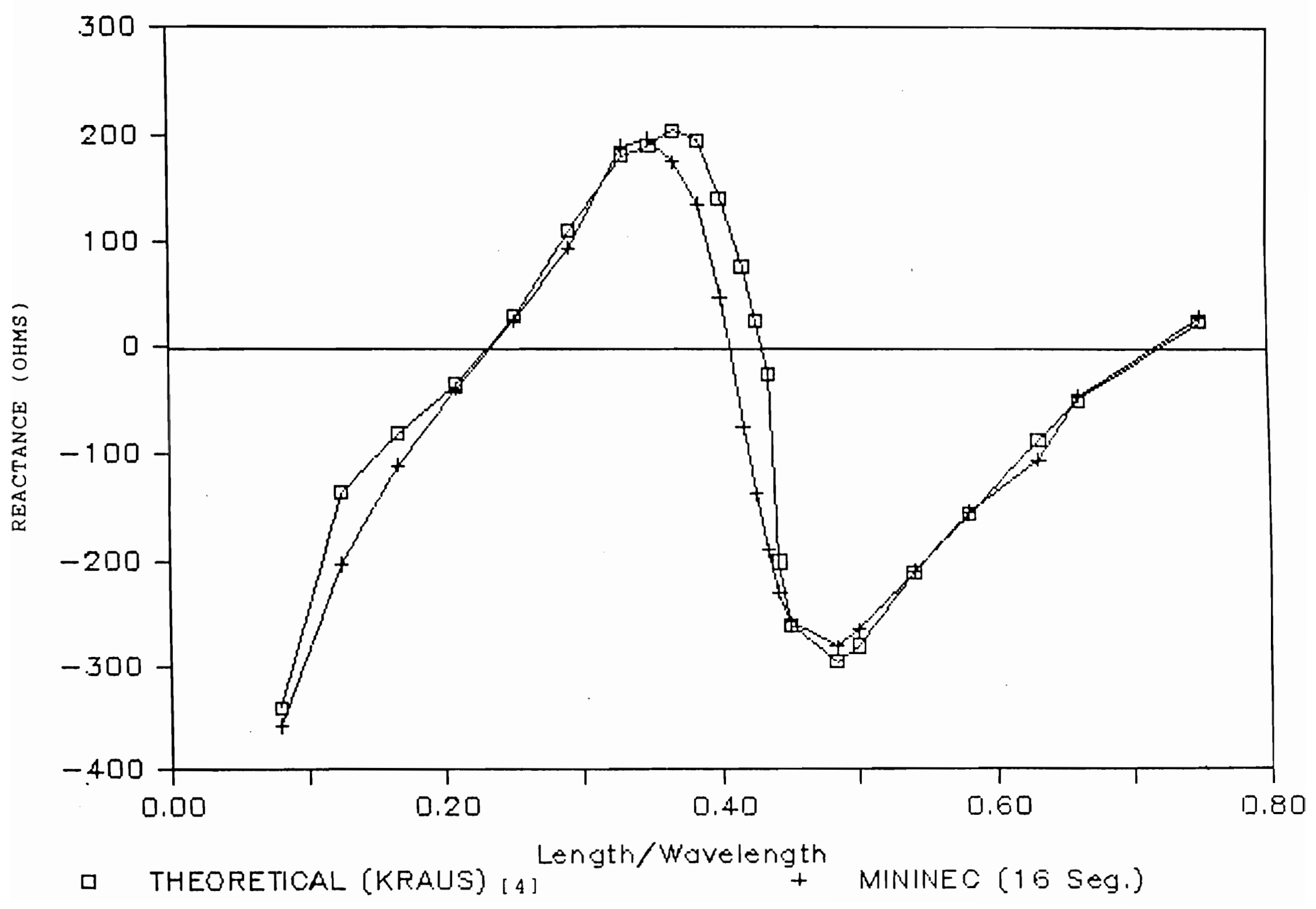


FIGURE 3.5(a): MONOPOLE INPUT RESISTANCE.

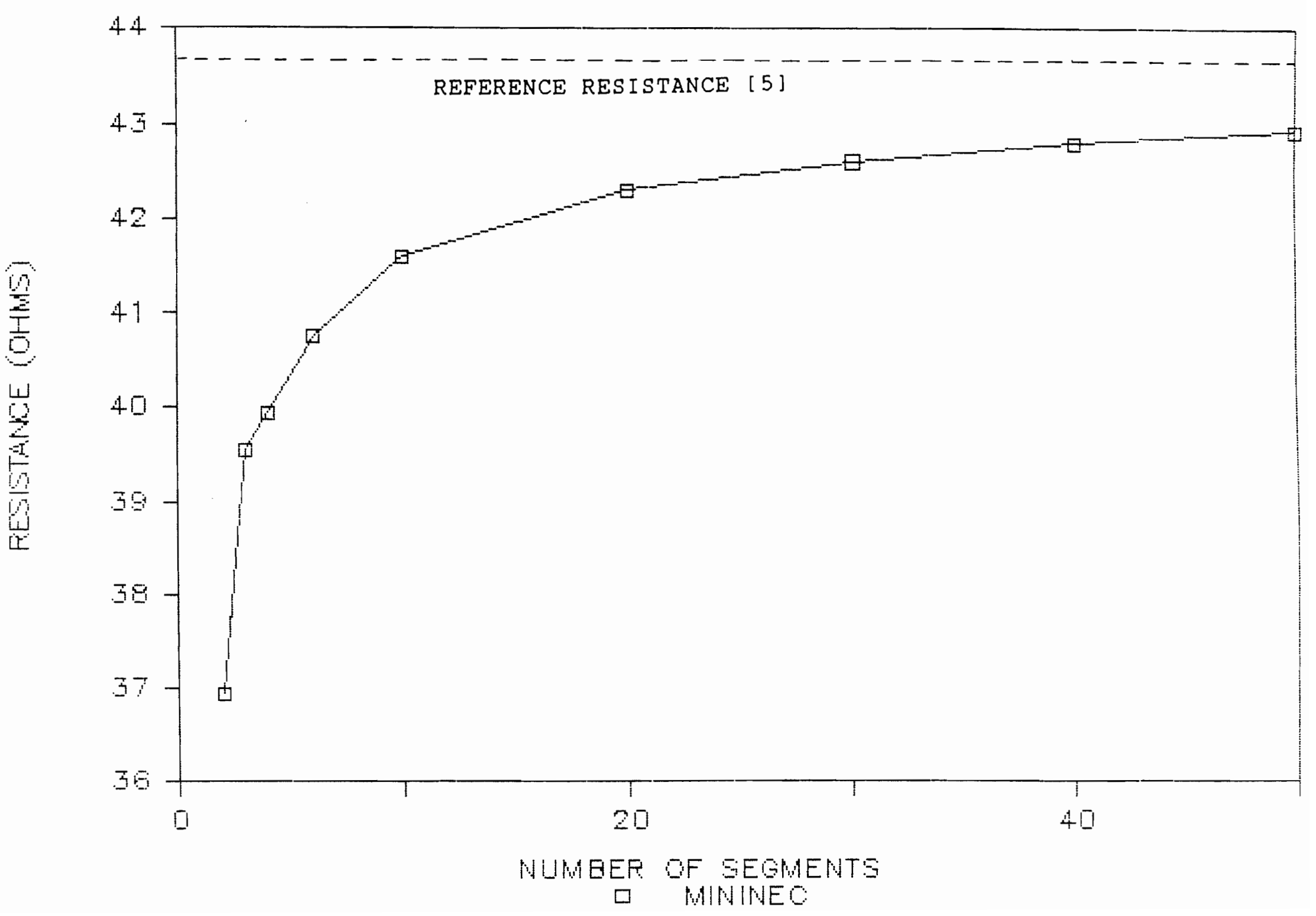


FIGURE $3.5(\mathrm{~b})$ : MONOPOLE INPUT REACTANCE.

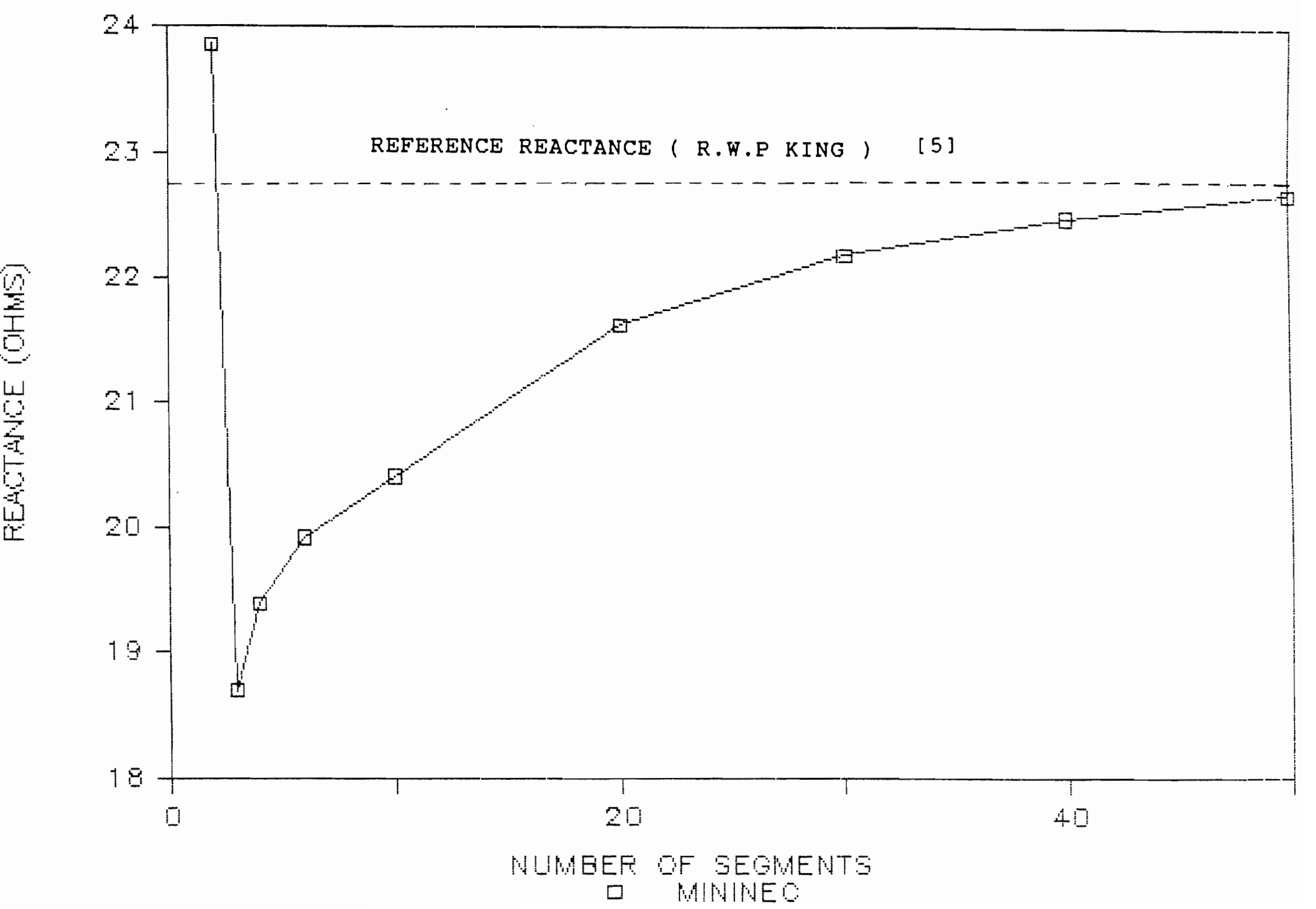


The convergence of the impedance as the number of segments is increased is shown graphically in Figure 3.5 .

Note that for a monopole with only 2 segments, the value of the reactance does not follow the convergence curve, whereas the resisce does follow the trend. This singularity is due to the fact that a very low segmentation density in MININEC, usually will yield erronous or inaccurate results. This will be shown in more detail in the next section, where more complicated geometries are analysed.

\subsection{MEANDER ANTENNAS}

\subsubsection{GENERAL:}

As a final proof of the validity of the program, it was necessary to analyse more complicated geometries. Meander antenna geometries were chosen because they obviously have a close relationship to the antenna design presented in this paper.

Meander antennas were first investigated by J. Rashed and Chen-To Tai in 1982 [3]. These antennas are a new type of reducedlength antennas. Previous works on the subject of reducing the size of a monopole while preserving the resonant frequency ended up in drawbacks such as bandwidth deterioration, pattern distorsion and reduced antenna efficlency.

Meander antennas are constructed from a continously folded wire intended to reduce the resonant length. Since the wire is folded three times over its course on each half section, the total length of the monopole is only one-third of the length of a monopole made from the same wire. Refer to to figure 3.6 .

The following parameters are defined for meander antennas:

- Reduction factor $B(<1)$ : If a conventional monopole of length $L$ and a meander monopole of length 1 , have the same resonant frequency, then the resonant factor is designated as:

$$
B=1 / L
$$

Equivalently, the resonant frequency of a meander monopole, fmo, is B times the resonant frequency fo, of a monopole with same length.

- Number of meander sections per wavelength, $\mathrm{N}$ :

This number serves to describe the geometry. A full meander section corresponds to $N=4$ while the geometry of figure 3.6 corresponds to $\mathbf{N}=2$. For a quarter-wavelength vertical monopole, this will normally coincide wires in the geometry. 
Experiments of reference [3] have shown that the reduction factor is of the order of 0.6 rather than one third. This factor depends primarily on the number of sections per wavelength $N$ and the width of the rectangular loops w. Also, experimental results show that antenna efficiency is comparable to that of an unfolded monopole.

Some measurements have been made with the separation of the wires, $w$, as a variable. These experiments show that when $w$ increases, the reduction factor B decreases, but the resonant resistance drops drasticalliy, these effects are also accompanied by a narrower bandwidth. Furthermore, for an increasing w, cross polarization appears in the plane $x z$. These aspects are further discussed in the next chapter.

3.3.2 MEANDER MONOPOLE ON CONDUCTING GROUND.

The Impedance of the monopole shown on Figure 3.6 , having an $N=6$, was compared with the results obtained by Rashed \& Tai [3]. the total length of the wire is $13.5 \mathrm{cmts}$. and has a value of $N=6$.

Furthermore, impedance computations were carried out for several segmentation schemes. It was established that for a total number of segments of less than 30 for this geometry, the program did not yield accurate results. Also for a segment number above 50, numerical errors ocurred. For segment numbers between 30 and 50 , the Impedance converges very well to the experimental value. The following table illustrates the results:

TABLE 3.2: MEANDER MONOPOLE IMPEDANCE VERSUS NUMBER OF SEGMENTS

\begin{tabular}{cc} 
NUMBER OF SEGMENTS & COMPUTED IMPEDANCE \\
\hline 24 & $114.8+j 5,469.8$ \\
35 & $19.2+j 11.4$ \\
42 & $19.6+j 15.2$ \\
49 & $19.8+j 17.4$ \\
51 & Error occurs.
\end{tabular}

REFERENCE INPUT RESISTANCE (Measured) : 20.5 Ohms.

It can be seen that the real portion of the computed Impedance converges very well to the measured value of the input resistance, at resonance.

The computations were all done for the same experimental resonance Erequency of $1050 \mathrm{MHz}$. 


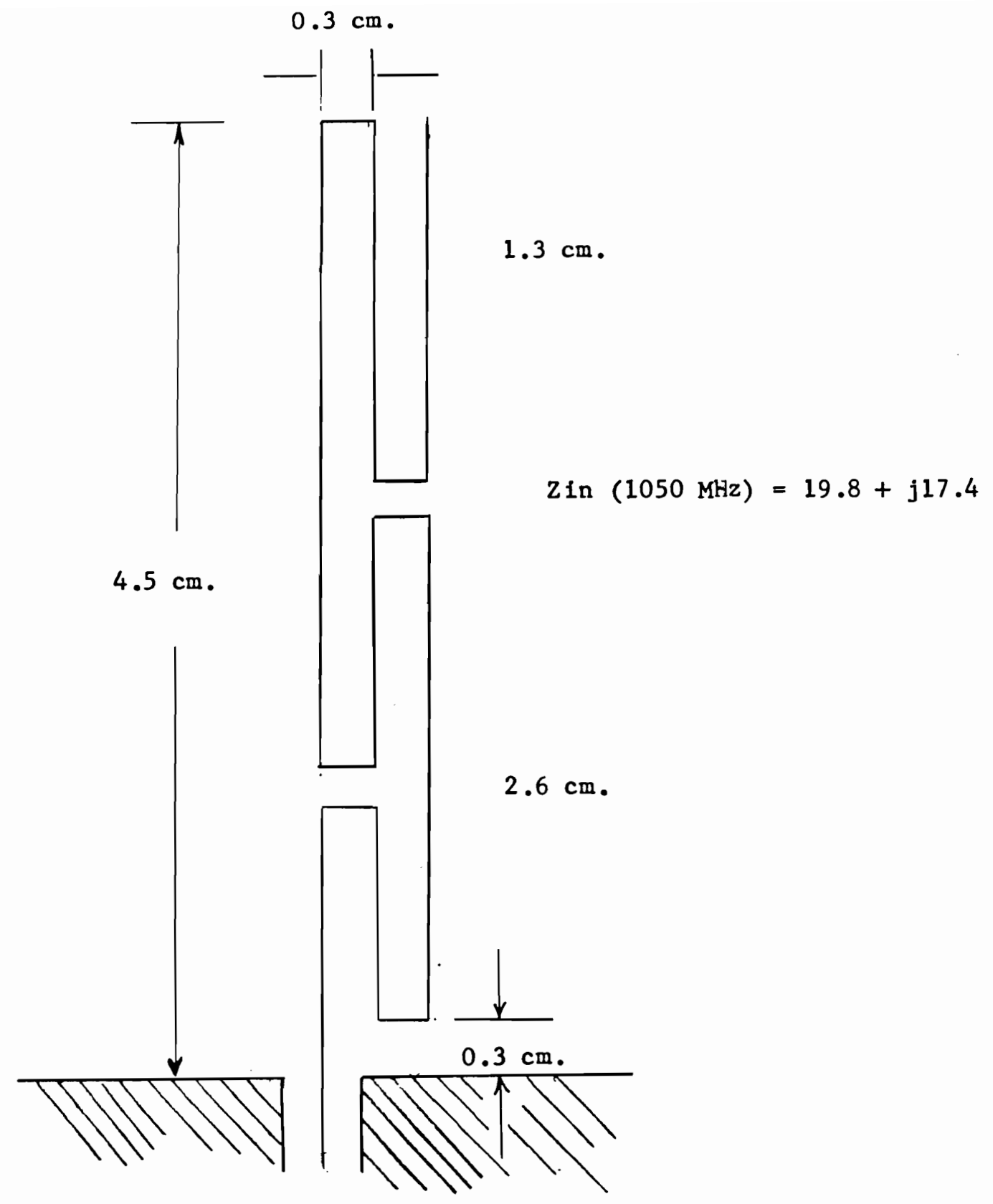

FIGURE 3.6: MEANDER MONOPOLE ANTENNA WITH $\mathbf{N}=6$. 
By the previous validation tests, it has been shown that MININEC, provides a rellable computer-based tool for analys is and synthesis of thin wire antennas. Since the antenna design described in the following chapters was based on the results obtained with this software, it was of primary importance to establish the validity of the program as a design tool. Furthermore, some of the program's limitations and characteristics were explored, that enabled the user to treat the results with enough confidence.

In particular, the segmentation density used for a specific geometry plays an important role in obtaining accurate results. The convergence testsfor various antennas reveals the accuracy that can be expected and provides a rational criterion for selection of segmentation density. Numerical problems may occur in the solution of a geometry when quantities become too small for the inherent accuracy of a computer. An example is the erronous results that can occur for very short segments (in terms of wavelength).

A practical limit for a semgent length seems to be about $10 \mathrm{E}-4$ wavelength, for a single precision 16-bit computer. 


\subsection{PRELIMINARY DATA ANALYSIS}

\subsection{GENERAL :}

This chapter will describe the preliminary analysis of known antennas, that led to the design of a folded meander dipole antenna covered in this paper. This antenna makes use of the reduction property of meander antennas, but has two important advantages over the simple monopole geometries of [3]

a- Folded dipole geometries can be designed to have a wide range of impedances, with careful choise of wire diameters and separation between the two branches of the dipole.

b- The size-reduction factor has been found to be typically less than 0.5. That is, this antenna has less than half the size of a folded dipole that has the same resonant frequency.

Preliminary analysis of simpler geometries such as meander dipoles and folded dipole antennas were accomplished as a means of obtaining sufficient reference data to accurately accomplish the subject design. These analysis, using MININEC are described in this chapter, as they provide in themselves sufficient explanation for some of the properties encountered in the final configuration.

Two known types of geometries were analysed using MININEC: meander monopoles antennas of reference (3) and the well-known folded dipole antenna. The analysis served the purpose of gathering some data on the behaviour and characteristics of these antennas, as well as to compare the results of MININEC with known theoretical or experimental data.

\section{2 MEANDER ANTENNAS}

\subsubsection{BACKGROUND}

The basics principles of meander antennas were covered in chapter 3. This section will describe with more detail some of the characteristics of these antennas, as obtained by modeling a basic meander monopole geometry, using MININEC.

As was previously said, meander antennas are made of a continously folded wire intended to reduce the resonant length of an antenna. Some of the main characteristics of the monopole meander antennas are summed : 
1- The reduction factor, defined as the ratio of the heigth $h$ of a meander monopole, to the length $L$ of a conventional monopole having the same resonant frequency, is of the order 0.60 to 0.7 . That is, the meander monopole is $30 \%$ to $40 \%$ smaller than a conventional monopole.

2- The input impedance is typicaliy less than that of a conventional monopole. It varles according to the number $N$ of meander sections per wavelength. This means that antenna has to be matched to the transmission line.

3- Antenna efficiency, by contrast to other size-reducing schemes, is similar to that of a conventional antenna.

other important characteristics were obtained my running MININEC with a simple meander monopole on conducting ground and $\mathrm{N}=2$. Some of the possible meander-monopole geometries with varying $N$ (number of meander sections per wavelength) are shown in figure 4.1

\section{2 .2 EFFECT OF $W$ ON ANTENNA CHARACTERISTICS}

The effect of $w$, the wire separation on meander antennas was analysed. The model used was a meander monopole on perfectly conducting ground, with a total wire length of $13.5 \mathrm{~cm}$. , a helgth of aproximately $4.5 \mathrm{~cm}$, and $\mathrm{N}=2$. . The wire radius was $0.4 \mathrm{~mm}$.

The results are shown in table 4.1 below. It can be seen that, as $w$ increases, the resonant resistance drops drastically, and the reduction factor $r$ decreases.

TABLE 4.1: EFFECT OF WIRE SEPARATION W ON CHARACTERISTICS OF MEANDER MONOPOLE $\mathrm{N}=2$, TOTAL WIRE LENGTH $=13.5 \mathrm{~cm}$.

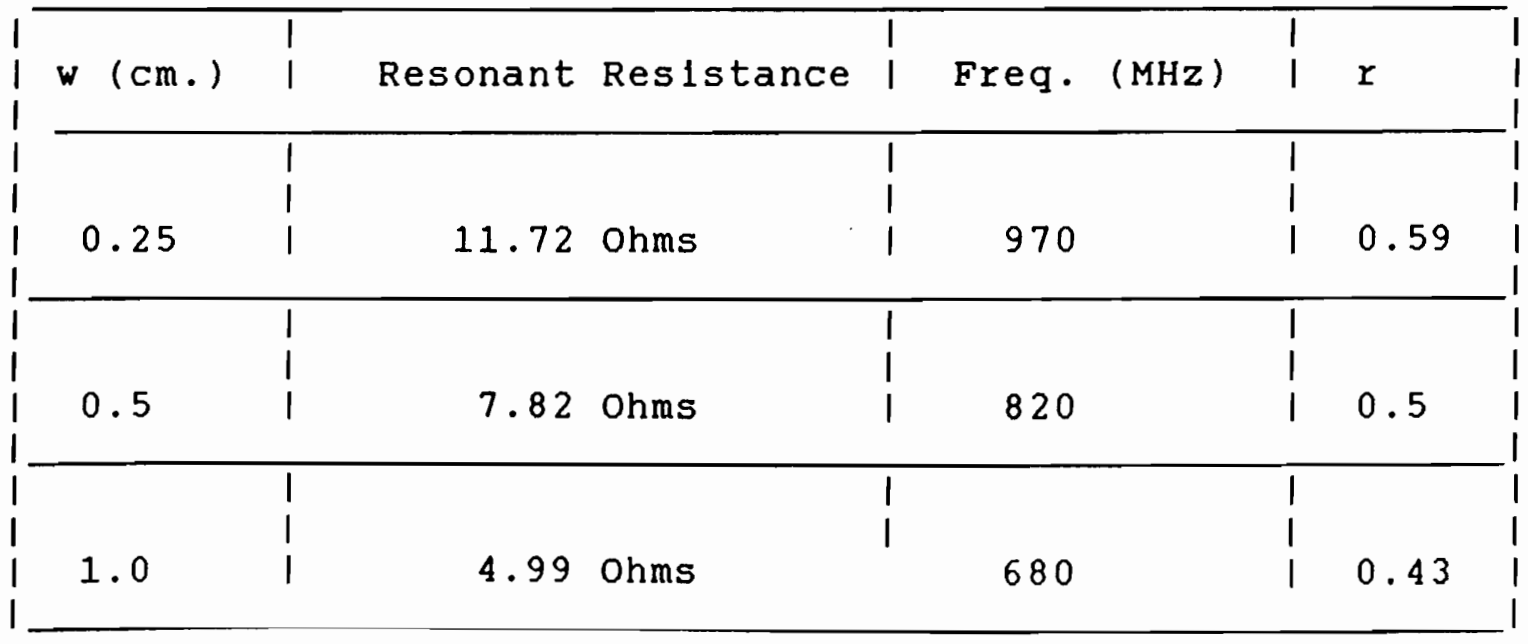




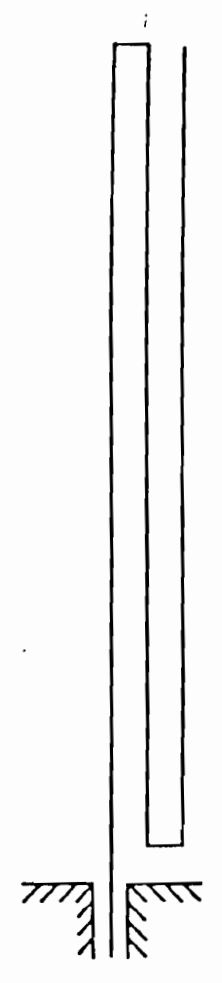

(a)

$\mathrm{N}=2$

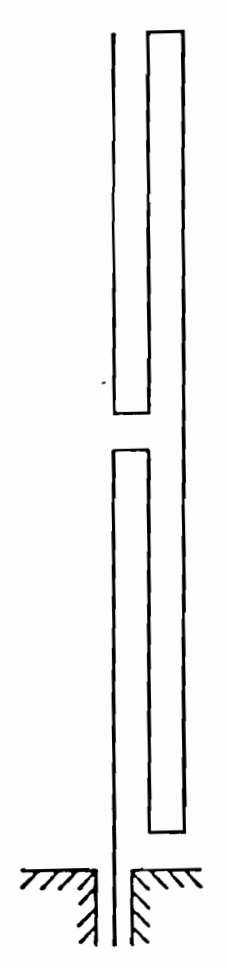

(b)

$\mathrm{N}=4$

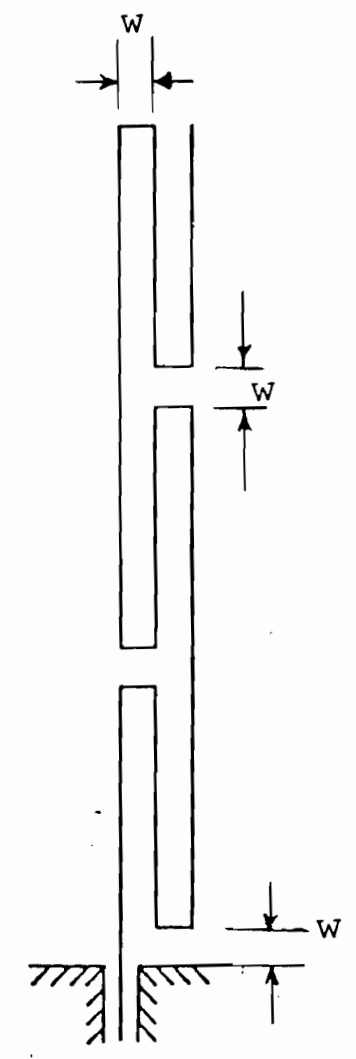

(c)

$\mathrm{N}=6$

FIGURE 4.1: SPECIAL CASES OF MEANDER MONOPOLES.

$(N$ is defined as the number of Meander sections per wavelength. For example, the monopole with $\mathrm{N}=4$ is composed of a full Meander section.) 
For each of the above antennas, with different wire separation $w$, the impedance was calculated for different frequencies. The bandwidth in each case was obtained using the following method:

1-Impedance values are normalized to the resonant resistance value, so that when plotted in a smith chart, they pass the center of the chart.

2- A circle corresponding to a VSWR $=2.0$ is used to find the upper \& lower frequencies.

The results are shown in table 4.2. Also the bandwidth as a percentage of the resonant frequency is shown for each case. This number is sometimes referred as the antenna "Q" factor.

It can be seen that, as w increases, the bandwidth tends to decrease accordingly. It is worth noting that the bandwidth of a meander monopole is considerably less than that of a conventional monopole. A reference monopole with the same resonant frequency would have a bandwith of about $10 \%$ its resonant frequency.

Flgure 4.2 shows the smith-chart plot of the meander monopole antenna impedances and their corresponding bandwidths.

Figure 4.3 is the smith-Chart for a conventional quarter-wavelength monopole.

TABLE 4.2: BANDWIDTH OF MEANDER MONOPOLES WITH N=2

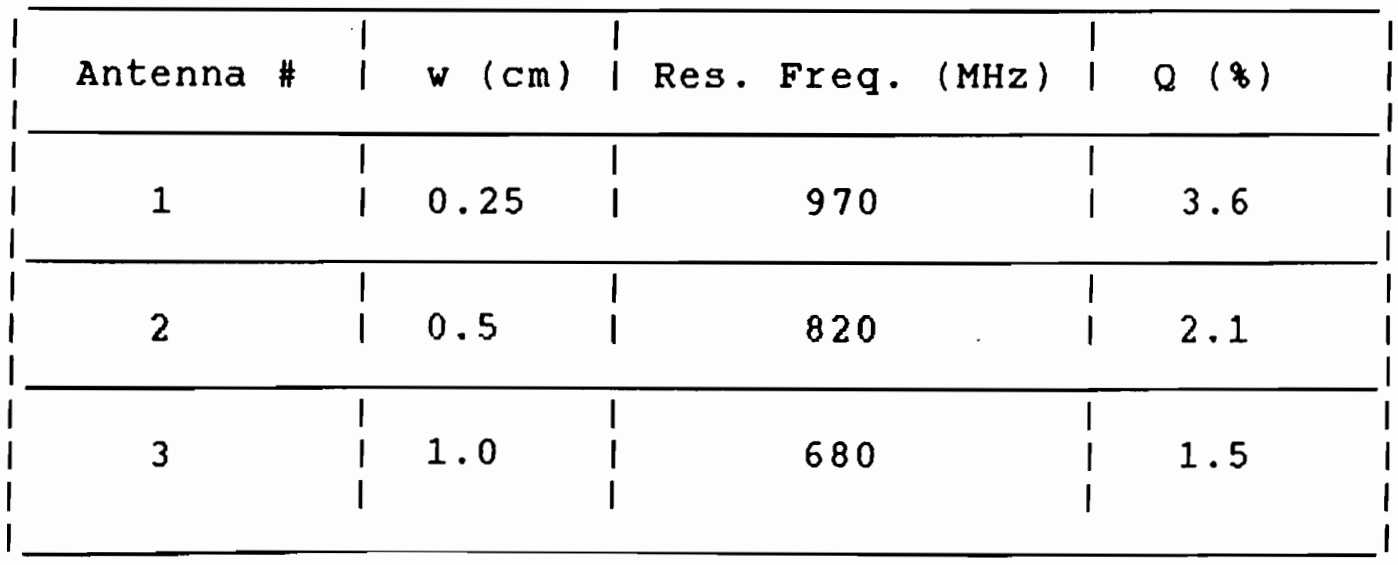




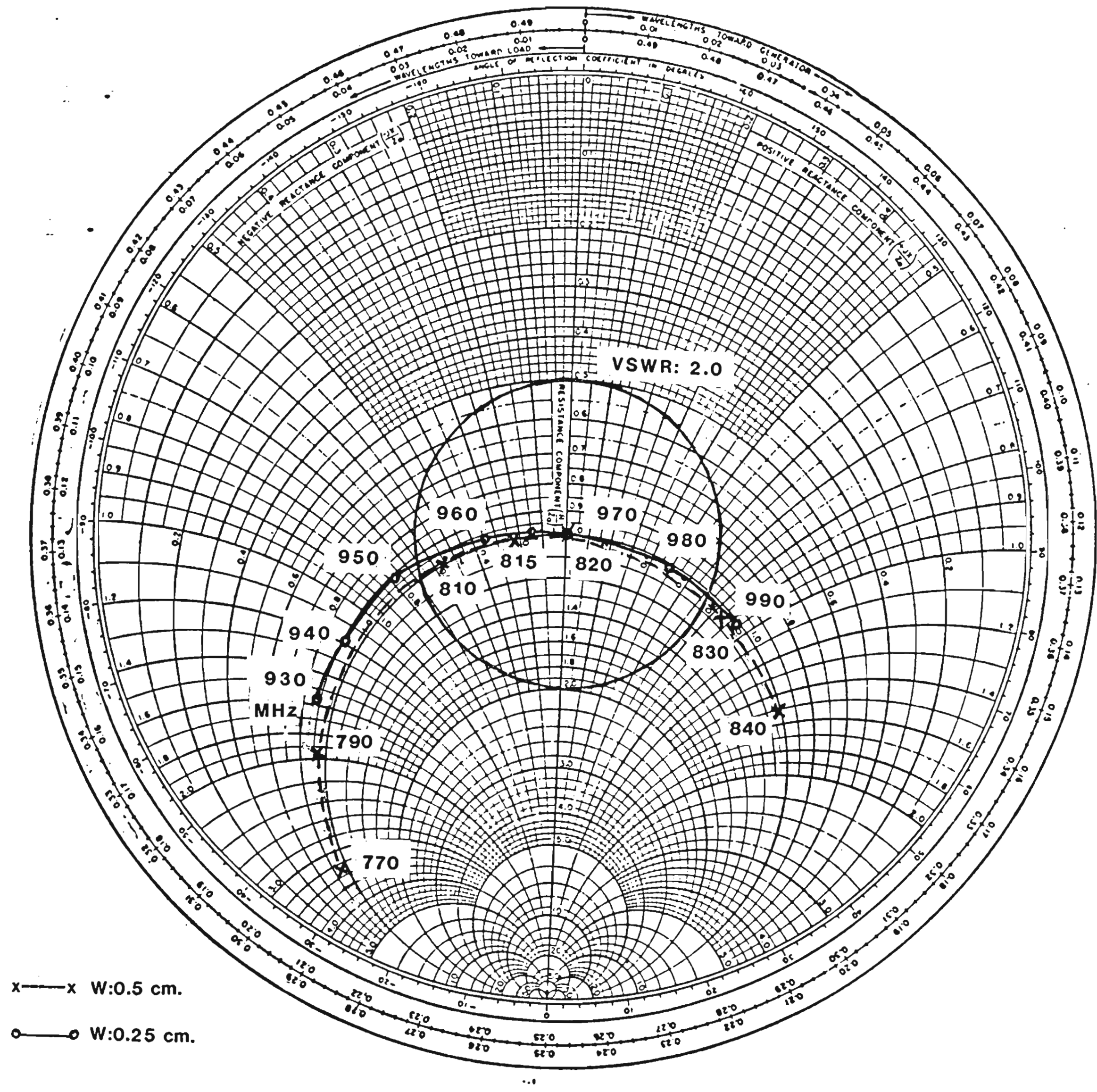

FIGURE 4.2 IMPEDANCE CURVE FOR MEANDER MONOPOLES $W I T H \quad W: 0.25$ AND:0.5 cm: CURVES ARE NORMALIZED TO THE RESONANT RESISTANCE VALUE. 


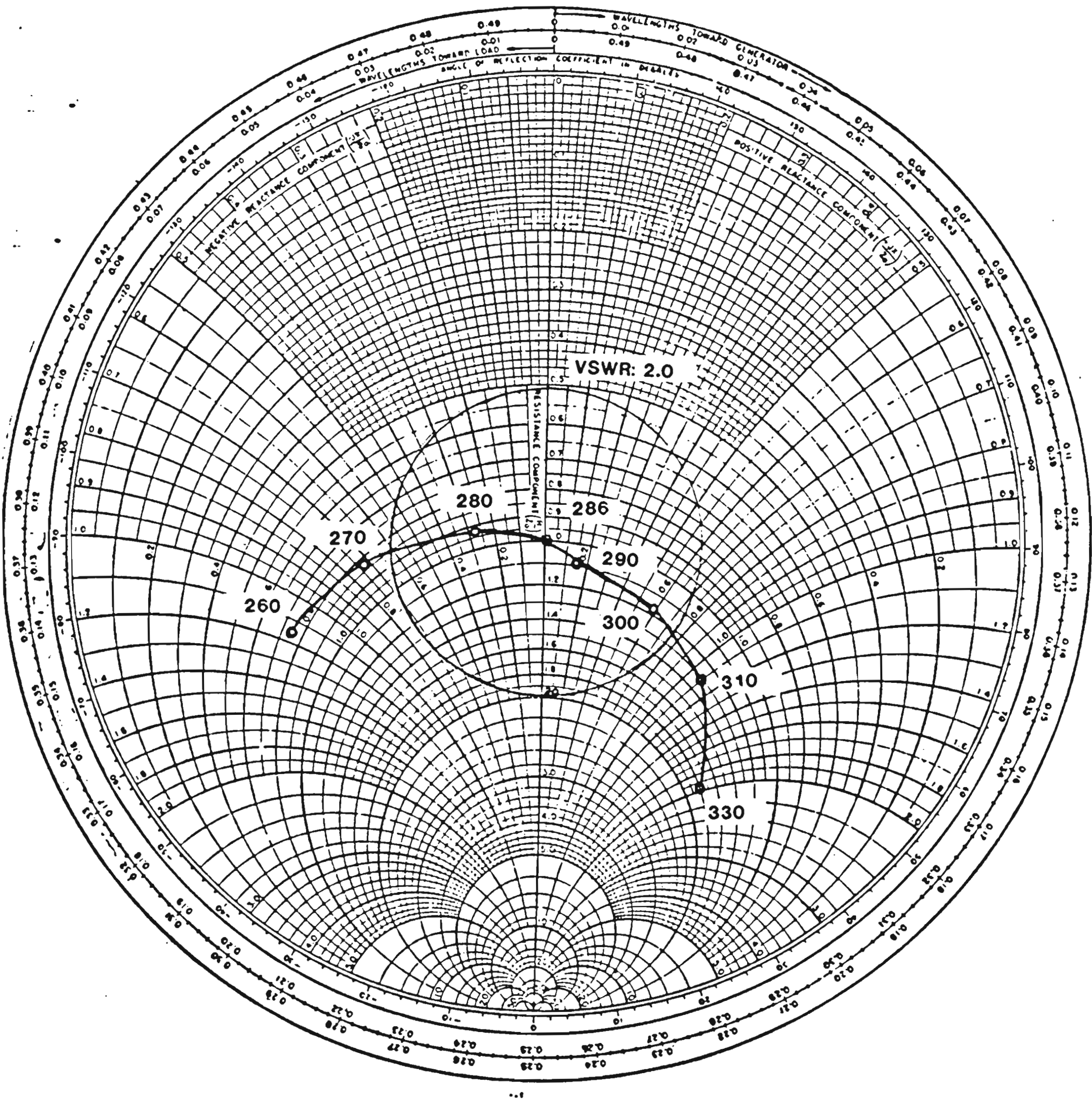

FIGURE 4.3: IMPEDANCE OF A CONVENTIONAL MONOPOLE OF LENGTH $=50 \mathrm{~cm}$.

Resonant Frequency is $286 \mathrm{MHz}$. 
The effect of $N$, or the number of meander sections per wavelength was analysed using MININEC. It was found that for a small constant $w$, an increasing $N$ means an increase in the reduction factor typically ranging from 0.6 for $\mathrm{N}=2$ to 0.7 for $\mathrm{N}=10$. However, one can decrease the reduction factor $r$ even further, by increasing $w$, as shown previously.

In general, the lower the $\mathrm{N}$, the better the size reduction property. But meanwhile, the resonant resistance and the bandwldth decrease as $r$ decreases. For the lowest value of $N$, i.e. $\mathrm{N}=2$ the reduction factor is 0.6 which corresponds to about 40 percent reduction in size. In this case, the resonant resistance is about 11 ohms, much lower than a conventional monopole.

The results are shown in table 4.3 :

TABLE 4.3: EFFECT OF $N$ ON ANTENNA CHARACTERISTICS TOTAL WIRE LENGTH $=13.5 \mathrm{CM}$.

$\mathrm{W}=0.25 \mathrm{CM}$.

\begin{tabular}{|c|c|c|c|c|c|c|}
\hline 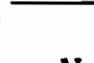 & 1 & & 1 & & 1 & Reduction \\
\hline $\mathbf{N}$ & 1 & fo( $(\mathrm{MHz})$ & 1 & Resonant $R$ (Ohms) & I & Factor $r$ \\
\hline 2 & $\begin{array}{l}1 \\
1 \\
1\end{array}$ & 970 & $\begin{array}{l}1 \\
1 \\
1\end{array}$ & 11.7 & $\begin{array}{l}1 \\
1 \\
1\end{array}$ & 0.59 \\
\hline 6 & $\begin{array}{l}1 \\
1 \\
1\end{array}$ & 1051 & $\begin{array}{l}1 \\
1 \\
1\end{array}$ & 20.1 & $\begin{array}{l}1 \\
1 \\
1\end{array}$ & 0.71 \\
\hline 10 & $\begin{array}{l}1 \\
1 \\
1\end{array}$ & 1113 & $\begin{array}{l}1 \\
1 \\
1\end{array}$ & 21.9 & 1 & 0.74 \\
\hline
\end{tabular}


Again, here we have a compromise between size-reduction and input resistance. In the case of the highest-resistance meander monopole $(\mathrm{N}=10)$, it would be possible to further increase the value of this resistance by increasing wire separation, to obtain a value of about 24 ohms. If this monopole were to be fed by a conventional 50-ohm coaxlal line, the antenna VSWR at resonance would be close to 2.0 .

This is a very acceptable value of VSWR, and the experimental monopoles of reference (3), yleld an efflciency comparable to that of a conventional monopole. However, is this loss of matching and level of complexity in the geometry, worth a mere 308 in size reduction? The answer, of course lies in the antenna application.

In the case of a monopole with an $N=2$, the reduction factor, again, can be reduced to less than 0.6 . The low resonant reslstance value can be matched to a conventional transmission line with a sleeve, without significant loss in efficiency.

This, of course represents a more viable solution, if the goal is to reduce the size of a monopole to roughly half its size.

\subsubsection{CURRENT DISTRIBUTION}

The current distribution of a meandermonopole with $\mathrm{N}=2$ was obtalned, as well as that of a convent1onal monopole. Flgure 4.4 shows both current distributions. The sample monopoles had their length of $13.5 \mathrm{~cm}$. divided into 38 segments, were mounted on a perfectly conducting infinite ground plane and were fed at the base by a one-volt source.

The curves show the current magnitude and phase variation versus segment number, with the voltage source being connected to segment number 1 .

Note that in general, the current values for the meander monopole are greater, reflecting the lower input resistance. Also, the meander monopole has its maximum current magnitude shifted from the excitation point, unlike the case of a conventional monopole. otherwise, the distribution follows the decreasing pattern of the conventional monopole, with the current at the end of the wire being equal to zero.

The phase in the current distribution curve for a meander monopole is seen to be relatively constant, having a variation not exceeding 2 degrees through the length of the antenna.

This contrasts with the conventional monopole that has a constantly decreasing phase. However, the overall phase varlation through the length of the wire seems to be too small to be consldered significant. 


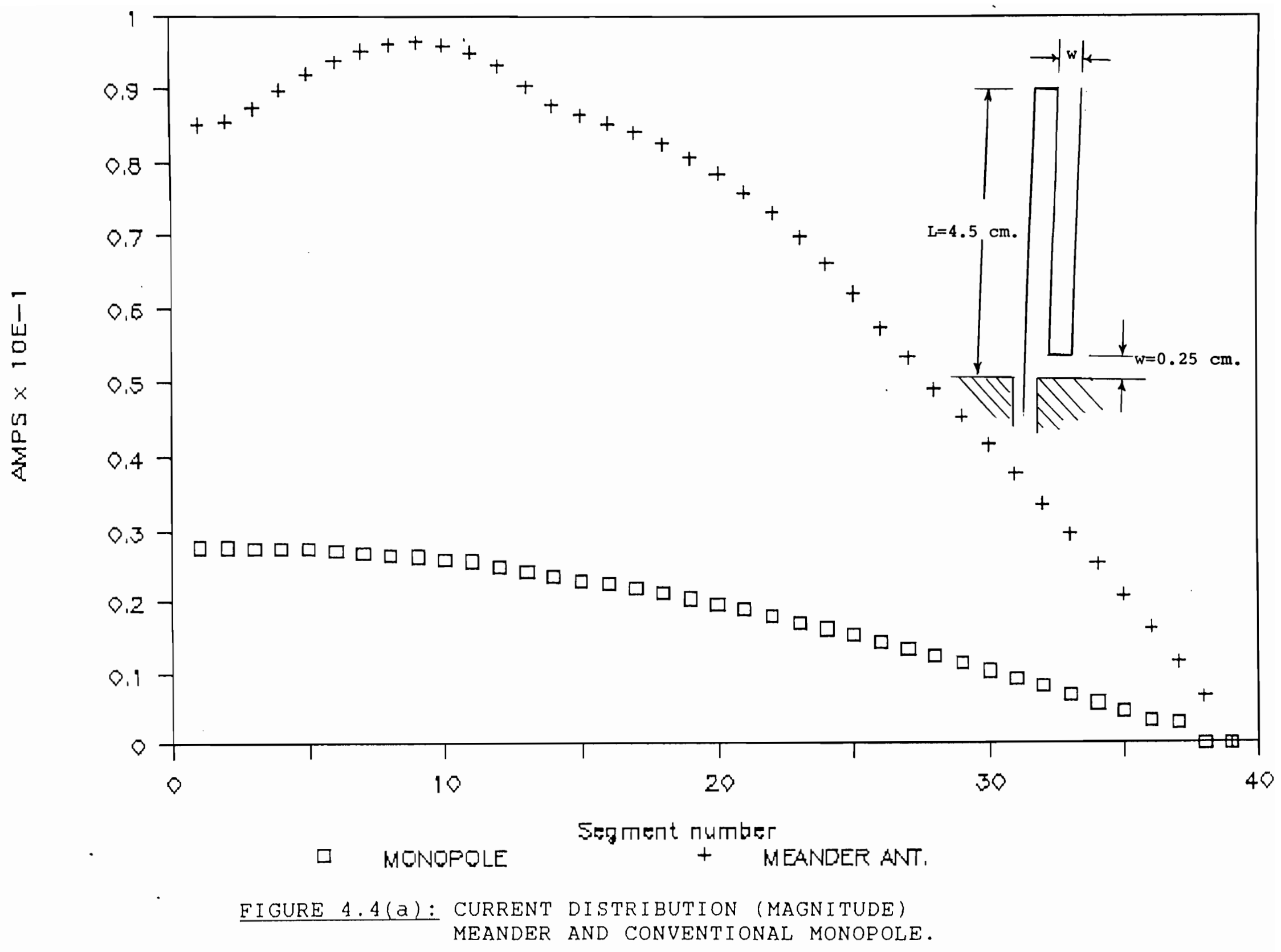




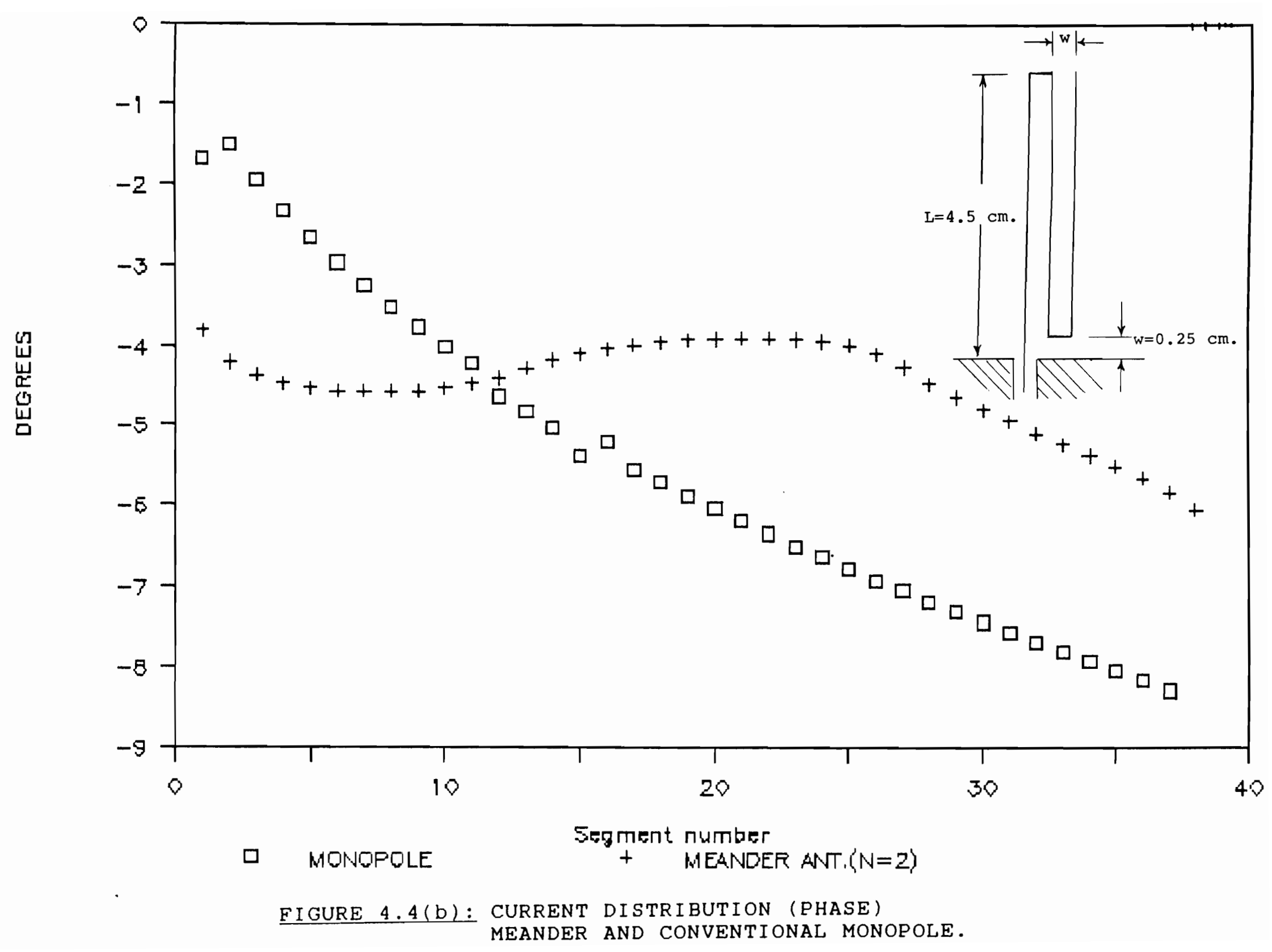


It is generally assumed that the current distribution of an infinitesimally thin antenna (length-to-radius equals infinity) is sinusoidal, and that the phase is constant over a half-wavelength interval, changing abruptly by 180 degrees between these intervals.

In the magnitude curve for the monopole, we can clearly see this trend, although only a quarter of the sinusoidal cycle can be seen, due to the antenna length. The phase for both the meander antenna and the monopole are almost constant, varying only a few degrees in both cases, reflecting the finite-diameter condition of the wires. The effect of finite-diameter wire and the ratio diameterto-antenna length is discussed in more detail in the following section.

\subsection{CYLINDRICAL ANTENNAS AND THE DIPOLE.}

\subsubsection{CYLINDICAL ANTENNAS:}

The concept of cylindrical antennas is not opposed to that of thin-wire antennas. Rather, it is a name given generally to wire monopoles or dipoles that have a length-to-wire radius ratio that is a finite quantity. A special case, of course is when the antenna is made of hollow pipe. The name "cylindrical" is used to reflect the fact that most theoretical work on dipoles is based on the assumption that the wire radius is very (infinetesimally) small compared to the antenna length. This assumption, although useful, is not accurate for real-life models, wire antennas having a finite length-to-diameter ratio have characteristics varying from the theoretical model, in accordance to the diameter of the conductor they are made of.

The 1mpedance characteristics of cylindrical dipoles have been extensively investigated [5] [6] [7]. Theoretical work has mainly been confined to thin antennas, with length-to-diameter ratio greater than 15. In most cases, the effect of the function connecting the radiating elements and the transmission line is usually not considered. Among various theories, the induced-emf method of computing the impedance of a cylindrical antenna based upon a sinusoidal current distribution is still found to be very useful. The formula derived from this method is extremely simple. It is, however, valid only when the half length of a center driven antenna is not much longer than a quarter wavelength. In practice, this is the most useful range.

To elliminate unnecessary computations, the formula has been reduced to the following form: 


$$
\mathrm{Z}_{1}=R(k 1)-j\left[120\left(\ln \frac{1}{\mathrm{a}}-1\right) \cot k 1-\mathrm{X}(k 1)\right]
$$

where:

$$
\begin{aligned}
\mathrm{Z} 1= & \text { input impedance of a center-driven cylindrical antenna } \\
& \text { of total length } 21 \text { and radius a. } \\
\mathrm{kl}= & 2 \pi(1 / \lambda) \text { i electrical length, corresponding to } 1, \\
& \text { measured in radians. }
\end{aligned}
$$

The functions $R(k l)$ and $X(k l)$ are shown in table 4.4 below, for the range $k l<\pi / 2$

TABLE 4.4: FUNCTIONS $R(k l)$ AND $X(k l)$ CONTAINED IN THE FORMULA OF A CENTER DRIVEN DIPOLE [6]

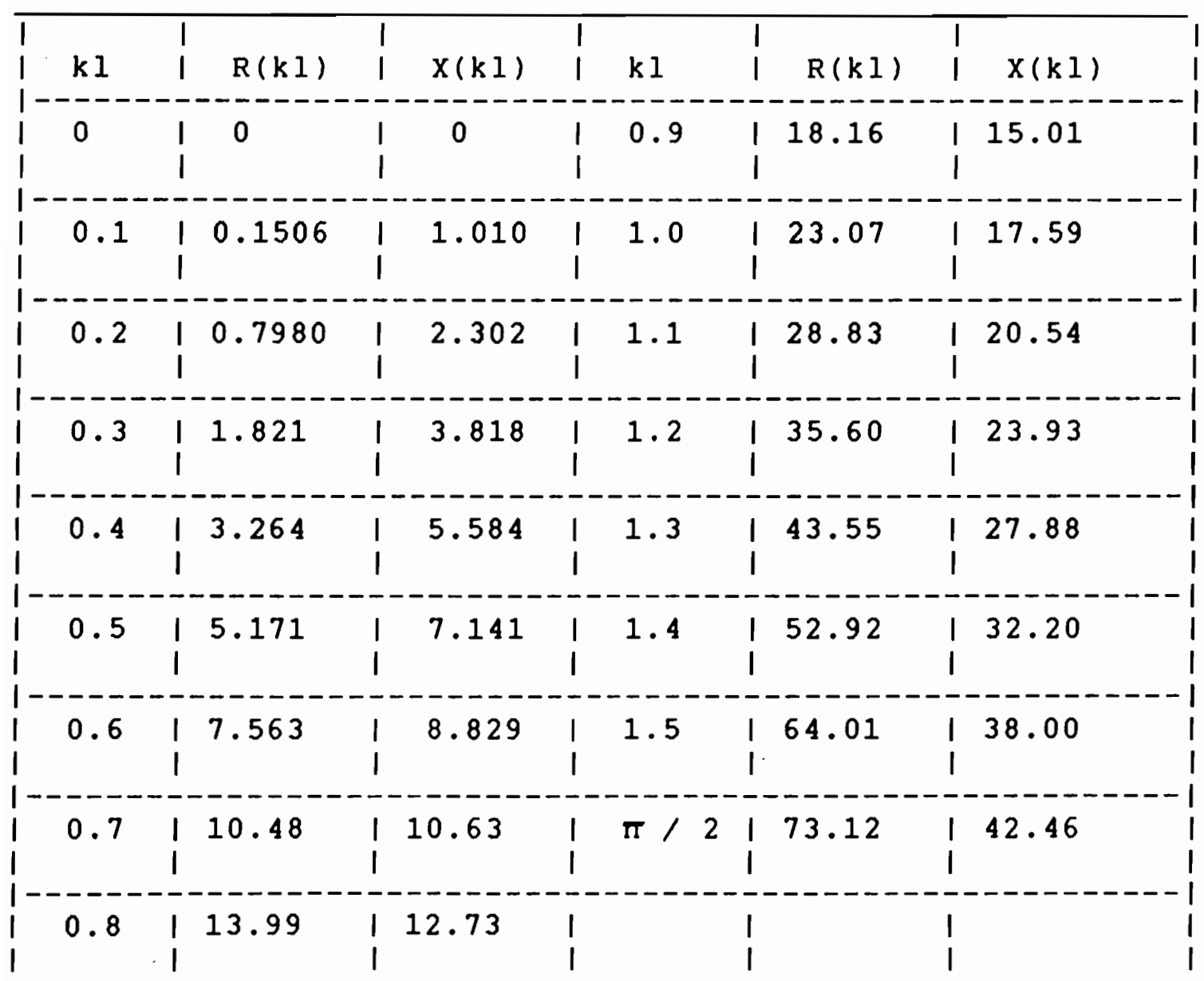


Note that for a kl equal to half wavelength, the impedance obtained is the known value for a half-wave dipole.

For antennas of half-length grater than half-wavelength, a number of refined theories provide formulas for the computation of the impedance function.

It should be emphasized that that all these theories are formulated by using an idealized model in which the terminal conditions are not considered.

\section{PRACTICAL EFFECT OF TERMINAL CONDITIONS}

In practice, the antenna is always fed by a transmission line. The effective terminal impedance of the line (often referred to as the antenna impedance) then depends not only upon the length and the diameter of the antennas, but also upon the terminal conditions.

In the typical case of a monopole on conducting ground, the impedance would also be a function of the size of the ground plane.

For a given terminal condition, the variation of the impedance of a cylindrical antenna as a function of the length and the diameter of the antenna is best shown in the work of Brown and woodward.(6) The data cover a wide range of values of the length-to-diameter ratio. Two useful sets of curves are reproduced in figures 4.5 (a) and (b).

The impedance refers to a cylindrical monopole driven at the base by a coaxial line though a large circular ground plane placed in the surface of the earth.

The arrangement is similar to that of the small quarter-wave monopoles mounted in the metallic roof of police-cars and ambulances.

In the figures, the length and the diameter of the antenna are measured in degrees, 1.e. a length of one wavelength is equivalent to 360 degrees. If the effects due to the terminal condition and finite-size ground plane are neglected, the impedance would correspond to one-half of the impedance of a center-driven antenna.

In using these data for design purposes, one must take into consideration the actual terminal conditions as compared to the condition specified by these two authors.

In general, MININEC will yield curves similar to the ones shown for values of length-to-diameter ratio relatively high. Ratios greater than 50, in particular, seem to satisfy the MININEC basic principle that the diameter be very small compared to the wavelength of the antenna.

The curves of Figures 3.3 and 3.4 were obtained for a length-todiameter ratio of about 100, using MININEC, and were used as a preliminary validation of the program for a simple monopole geometry. 


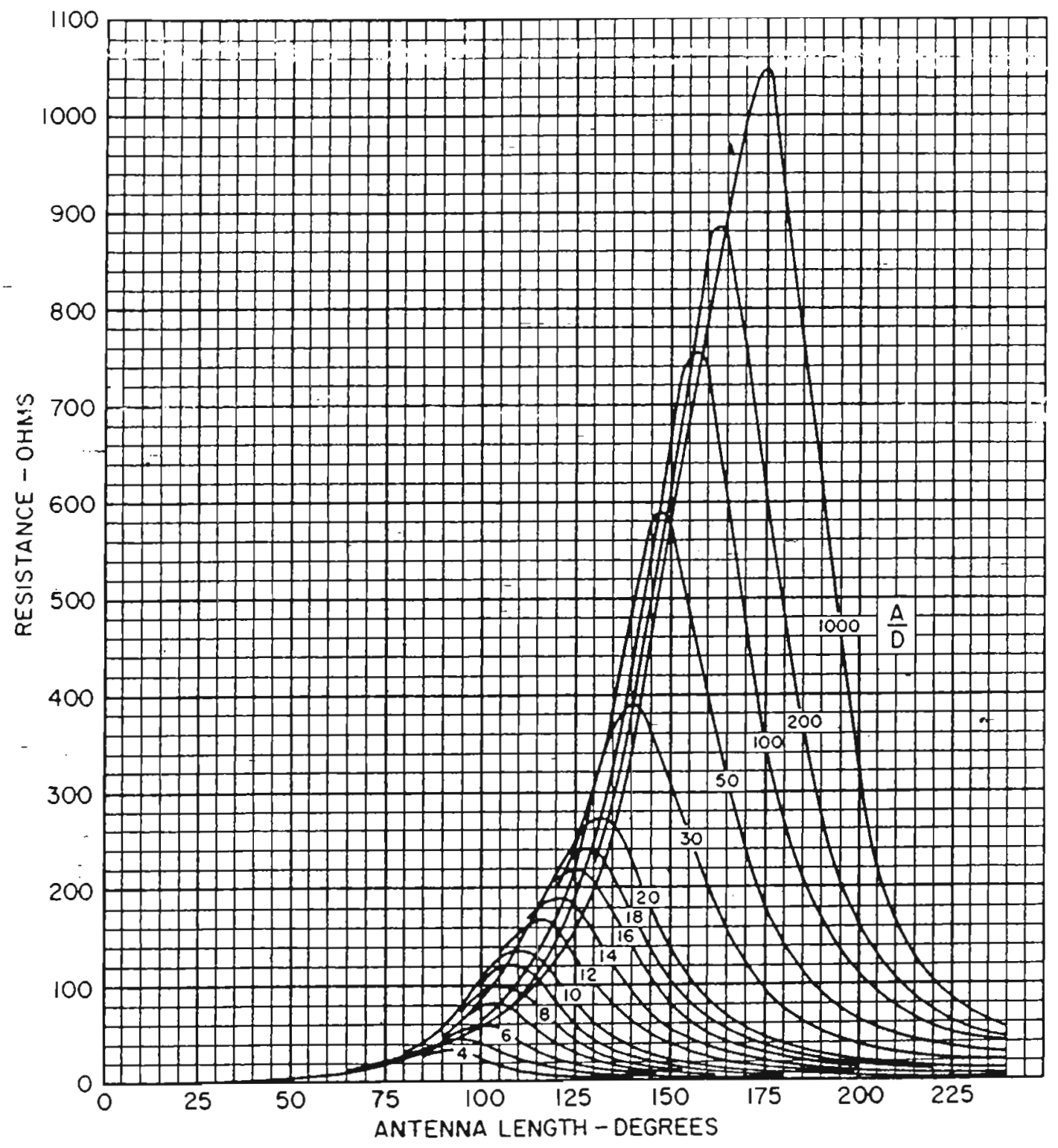

FIGURE $4.5(a)$ : INPUT RESISTANCE OF MONOPOLE OVER CONDUCTING GROUND, FOR DIFFERENT DIAMETER TO LENGTH RATIOS (A/D) VERSUS ELECTRICAL LENGTH. [9] 


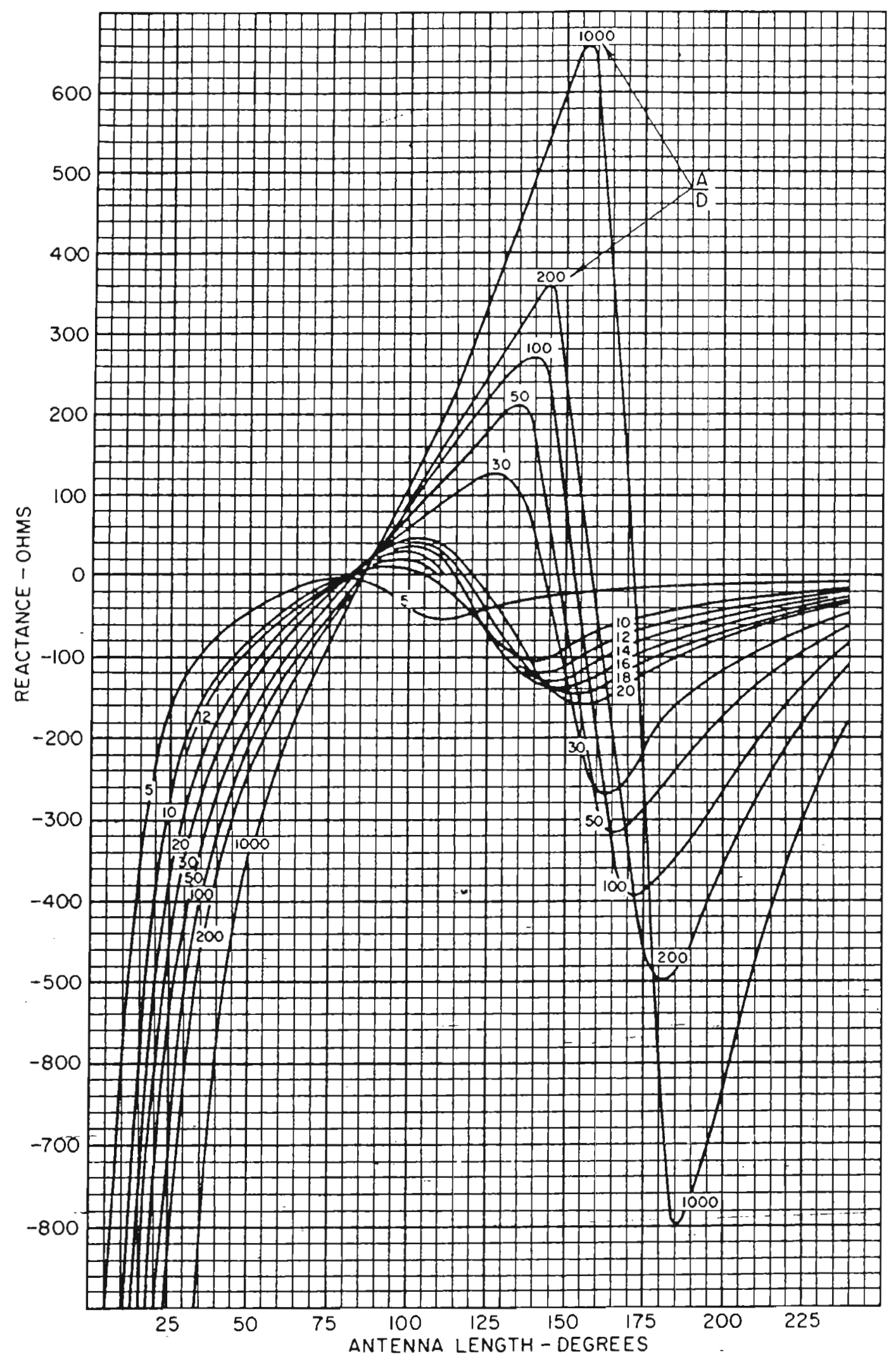

FIGURE $4.5(\mathrm{~b})$ : INPUT REACTANCE OF MONOPOLE OVER CONDUCTING GROUND FOR DIFFERENT DIAMETER TO LENGTH RATIOS (A/D) VERSUS ELECTRICAL LENGTH [9] 
The dipole antena is one of the simplest and most extensively used antennas. There exists enough literature explaining the theoretical aspects of dipole antennas, to try to cover them here. However, it is important to mention some practical considerations of dipole antennas that have direct relation to the design at hand.

\section{LENGTH OF A RESONANT DIPOLE ANTENNA}

The resonant length of a dipole antenna at a given frequency is deflned as the length at which the $1 \mathrm{mpedance}$ of the antenna is purely resistive. This is analogous to a tuned circuit; at the resonant frequency the input impedance of the circuit is a resistance. Unlike the case of an open-circulted transmission line the resonant lengths of a dipole antenna do not occur at at exact multiples of a half-wavelength. For example, a dipole of exactly a half-wavelength long has an input impedance of: [5]

$$
\mathrm{z}_{\text {in }}=\mathrm{R}_{\text {in }}+J \mathrm{x}_{\text {in }}=73+f 42.5 \text { ohms. }
$$

Practicaily, if we reduce the length slightly, the half-wave dipole can be made to resonate with $x i n=0$. The amount of shortening required to achleve this depends on the diameter of the antenna. As the antenna becomes thinner the shortening factor tends to one, that is, no change in length is required. For a typical dipole having a wire radius-to-length ratio of about 100 , the shortening factor is close to 0.96 . This means that the real resonant length for this case, would be 0.96 of a half-wavelength. [5]

It should be noted that, as the length is reduced to achieve resonance, the input resistance also decreases.

For practical purposes, however, a factor of 0.96 to 0.98 can be used for most thin wire dipoles. The input resistance will be in the order of 60 to 65 ohms.

Figure 4.6 shows the factor by which the length of the dipole in free space should be multiplied to to obtain the physical length of a half-wave dipole, as a function of the ratio of half-wavelength to conductor diameter. 


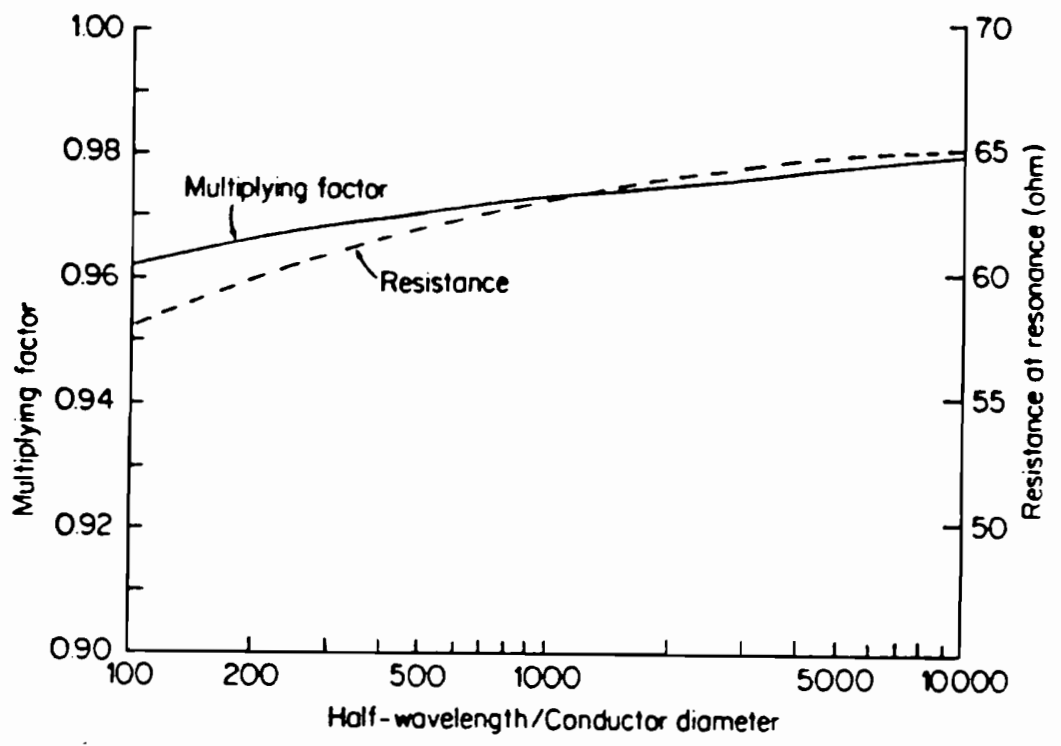

FIGURE 4.6: RESONANT RESISTANCE OF HALF-WAVELENGTH DIPOLE AND LENGTH FACTOR VERSUS HALF-WAVELENGTH TO WIRE-DIAMETER RATIO [10]

\section{BANDWIDTH OF A DIPOLE:}

The IEEE Standard Dictionary of Electrical and Electronic terms defines the bandwidth of an antenna as "the range of frequencles within which the performance of the antenna, with respect to some characteristics, conforms to a specified standard." [9] The characteristics referred to can be pattern or impedance. For the latter, which is usually more sensitive to frequency, a useful standard is that the voltage standing wave ratio be less than 2.0 . If the antenna impedance is $\mathrm{Za}$ and the characteristic impedance of the transmission line feeding the antenna is $\mathrm{zo}$ then the voltage reflection coefficient $p$ is:

$$
\mathrm{p}=(\mathrm{za}-\mathrm{zo}) /(\mathrm{za}+\mathrm{zo})
$$

The VSWR is related to $p$ by:

$$
\text { VSWR }=s=(1+|\mathrm{p}|) /(1-|\mathrm{p}|)
$$

From transmission line theory, the percentage of reflected power is equal to:

$$
\text { P. reflected }=|p| *|p| * 100
$$

And the percentage of transmitted power is equal to:

$$
\text { P. } x \text { itted }=(1-|p| *|p|) * 100
$$

A VSWR of 2.0 corresponds therefore to a reflected power of $11.1 \%$ 
Consider a dipole antenna of resonant length connected to a transmission line that has a characteristic impedance equal to the input Impedance of the antenna at resonant frequency. The resistance change in the region around resonance is relatively small compared to the change around in reactance. Consequently, the princlpal cause of the antenna change in VSWR is the reactive component of the antenna impedance when the frequency is varied. An antenna that has a relatively slow rate of change in diameter wlll therefore have a wider bandwidth. Figure 3.5 (b) shows that an antenna with a larger diameter-to-length ratio will have a wider bandwidth compared to an antenna with a smaller diameter-to-length ratio.

The following flgure shows the Voltage standing wave Ratio (VSWR) as a function of frequency on a 72 ohm transmission line feeding two dipoles of length 0.5 meter having two values of wire radius a.

Note that for the dipole of radius $a=0.005 \mathrm{~m}$. the resonant frequency is $285 \mathrm{MHz}$ and the bandwidth is about $16 \%$.

For the thinner dipole with radius $a=0.0001$, the resonant frequency is $294 \mathrm{MHz}$ and the bandwidth is about $8 \%$

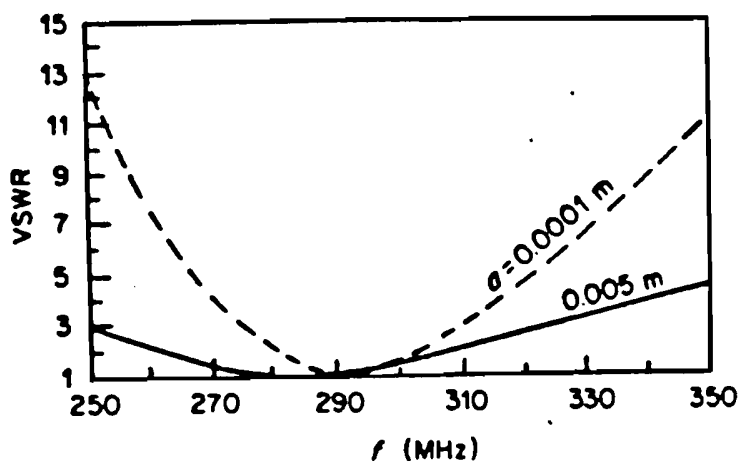

FIGURE 4.7 : VSWR versus frequency on a 72 ohm transmission line feeding two dipoles of $0.5 \mathrm{~m}$. length having two values of wire radius a. [9] 


\subsection{THE FOLDED DIPOLE:}

\subsubsection{THEORETICAL ANALYSIS:}

This section will describe the theoretical principles underlying the characteristics of a folded dipole. The theoretical analysis is important in understanding some of the features of our final design.

A folded dipole is formed by joining two cylindrical dipoles at the ends and driving them by a pair of transmission lines at the center of one arm as shown in figure 4.8. The diameters of the two arms can be either identical or different. A simple analysis based upon a quasi static approach of the operation of a folded dipole has been given by Uda and Mushlake [8].

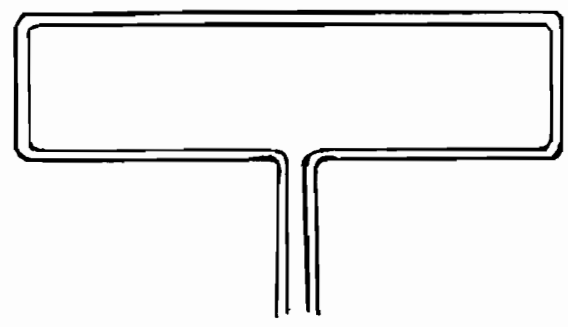

FIGURE 4.8 Folded Dipole.

According to their method, the excitation of a folded dipole can be considred as as a superposition of of two modes of operation, as shown in flgure 4.9. As can be seen, there is a symmetrical mode, characterized by two equal driving voltages, and an asymmetrical mode, characterized by equal and opposite currents on the the two arms of the folded dipole.

The impedance of the symmetrical mode can be calculated by making use of the equivalent radius of two conductors.

As far as the impedance characteristics and radiation pattern are concerned, a thin cylindrical antenna with a non-circular crosssection behaves like a circular cylindrical antenna with an 


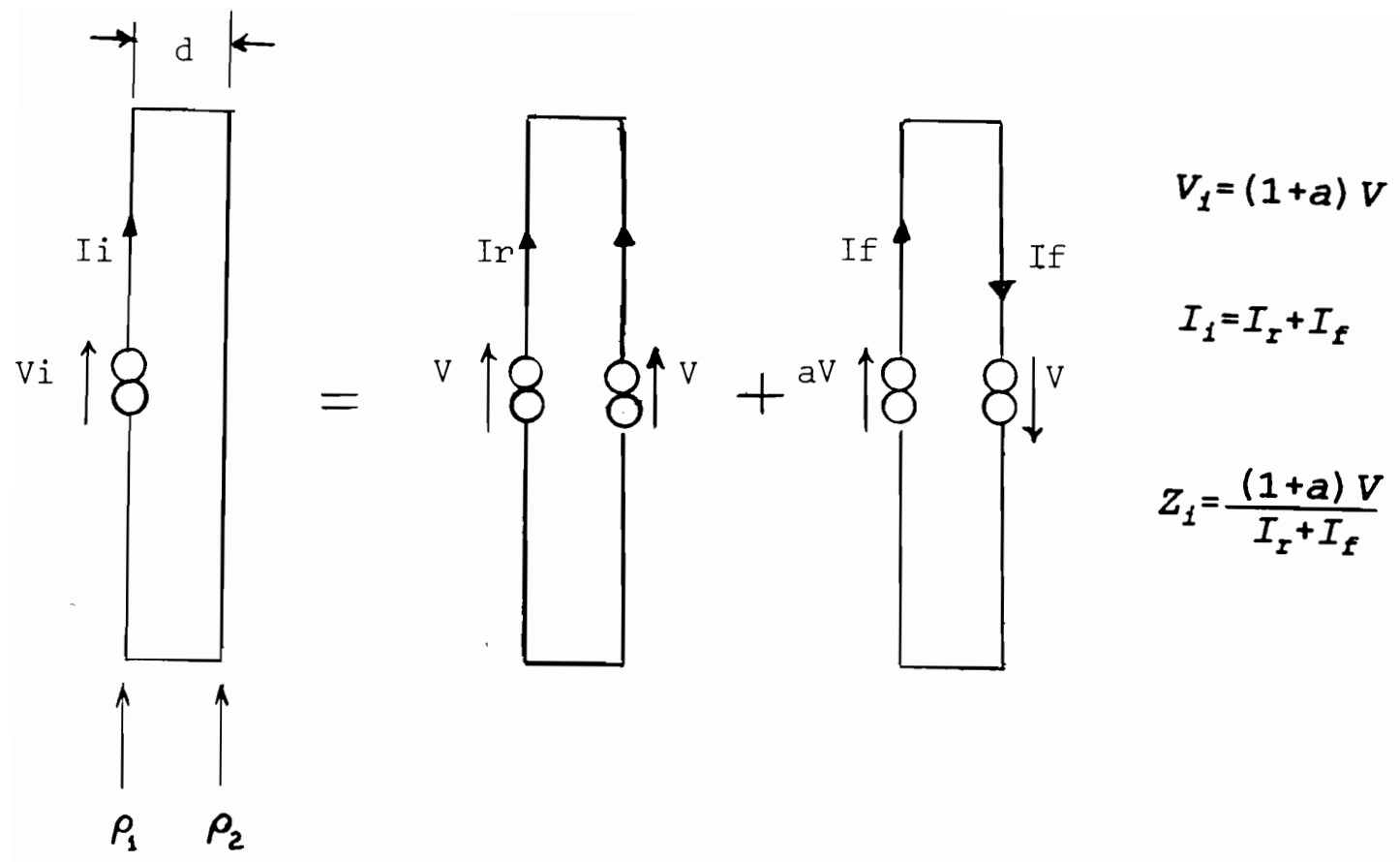

a) Decomposition of the folded dipole into two fundamental modes.

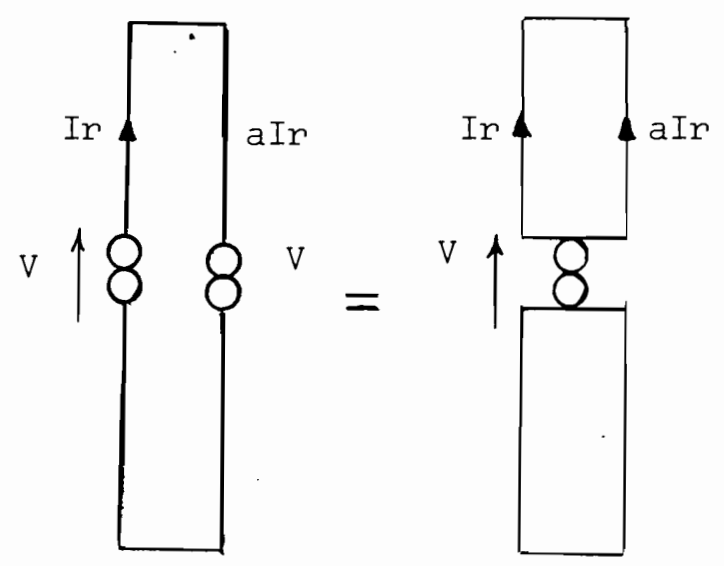

b) Equivalent representation of the symmerical mode in computing $\mathrm{Zr}$. 
equivalent radius. In stating this characteristics, the terminal effect, is of course, not considered. The equivalent radius of many simply shaped cross sections can be found by the method of conformal mapping [9]. For an elliptical cross-section the following simple relation exists :

$$
\text { Aeq }=1 / 2(a+b)
$$

where: $\quad a=$ major axis of ellipse.

$b=$ minor axis of ellipse.

For a rectangular cross section, the result is plotted in figure 4.10

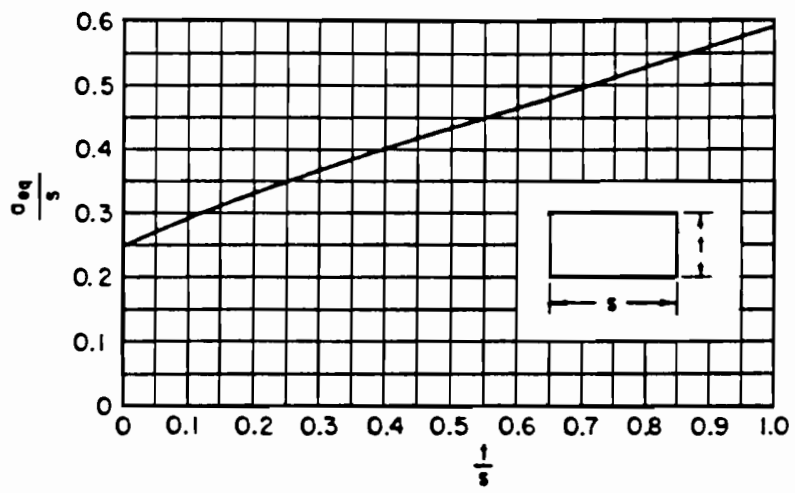

FIGURE 4.10: EQUIVALENT RADIUS OF A RECTANGLE AS A FUNCTION OF THE RATIO OF THICKNESS $t$ TO WIDTH $S$ [6]

The equivalent radius of parallel cylinders of radius $\rho_{1}, \rho_{2}$ and separated by a distance d between the centers is given by:

$$
\ln p_{0}=\frac{1}{\left(p_{1}+p_{2}\right)^{2}}\left(p_{1}^{2} \ln n_{1}+\rho_{2}^{2} \ln p_{2}+2 p_{1} p_{2} \ln \alpha\right)
$$

This of course, represents the case of a folded dipole, where the radil of the two conductors composing the arms of the dipole are both finite quantities. 
The symmetrical component of a folded dipole, can therefore be represented by a cylindical dipole (see fig. 4.9 - b) with an equivalent radius pe given by:

$$
\ln \rho_{e}=\ln \rho_{1}+\frac{1}{(1+\mu)^{2}}\left(\mu^{2} \ln \mu+2 \mu \ln v\right)
$$

where the parameters are:

$$
\begin{gathered}
a=\frac{\cosh ^{-1} \frac{\gamma^{2}-\mu^{2}+1}{2 \gamma}}{\cosh ^{-1} \frac{\gamma^{2}+\mu^{2}-1}{2 \gamma \mu}} \\
\mu=\rho_{2} / \rho_{1} \\
\gamma=\alpha / \rho_{1}
\end{gathered}
$$

The impedance of the asymmetrical mode, characterized by equal and opposite currents on the two arms is the same as the shorted section of a transmission line of length equal to 1 ; that is:

$$
\mathrm{Z}_{f}=\frac{(1+a)}{2 I_{f}}=j \mathrm{Z}_{o} \tan k I
$$

where $z_{0}$ is the characteristic impedance of the two-wire line. Expressed in terms of $\mathrm{Zr}$ and $\mathrm{Zf}$, the impedance of a folded dipole is given by:

$$
\mathrm{Z}=\frac{V_{1}}{I_{1}}=\frac{(1+a) \bigvee}{I_{Y}+I_{f}}=\frac{2(1+a)^{2} Z_{Y} Z_{f}}{(1+a)^{2} Z_{I}+2 Z_{t}}
$$


An equivalent clrcult of a folded dipole, based upon the previous formula is shown in figure 4.11 .

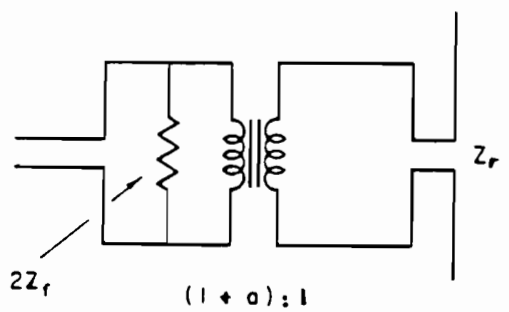

FIGURE 4.11: EQUIVALENT CIRCUIT OF A FOLDED DIPOLE [6]

\subsubsection{IMPEDANCE TRANSFORMATION AS A FUNCTION OF CONDUCTOR SIZES:}

The step-up impedance ratio shown in figure 4.11 as a function of $\mu$ and $\gamma$ has been calculated, by using the formula for the parameter $a$, given in the previous section. (4.10)

When $\rho_{1}$ and $\rho_{2}$ are small compared with $d$, the wire separation, the value of a is given to a good approximation by:

$$
a=\ln \left(d / p_{1}\right) / \ln \left(d / p_{2}\right)
$$

The step-up transformation ratio is an important property of folded dipoles, and in fact is what makes this antenna atractive : the possibility of obtaining almost any desired impedance, by a careful choise of the wire radil and the separation d betw en the arms.

The formulas described in this section have been tabulated for use by antenna designers. Figures 4.12 and 4,13 give two representations of the transformation ratio $(1+a)^{2}$ in a logarithmic scale as a function of the ratio between wire radi1. Note that in figure 4.12 for identical wire radius on both arms, the step-up ratio is 4.0 for all wire separations. This reflects the known value of a folded dipole: 300 ohms or about four times the impedance of an open dipole. 


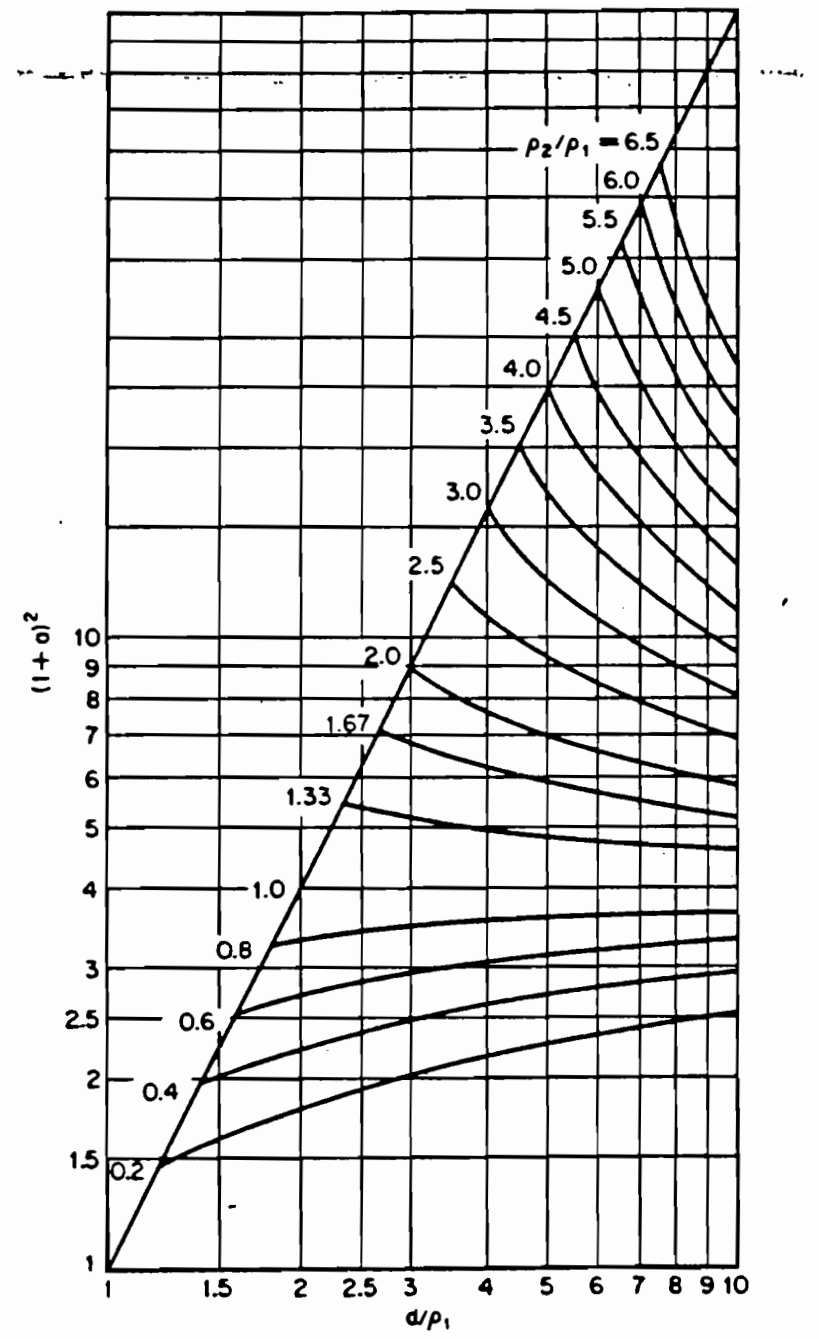

FIGURE 4.13: IMPEDANCE TRANSFORMATION RATIO VERSUS DIAMETER RATIO AND $\mathrm{d} / p_{1} \cdot[6]$ 


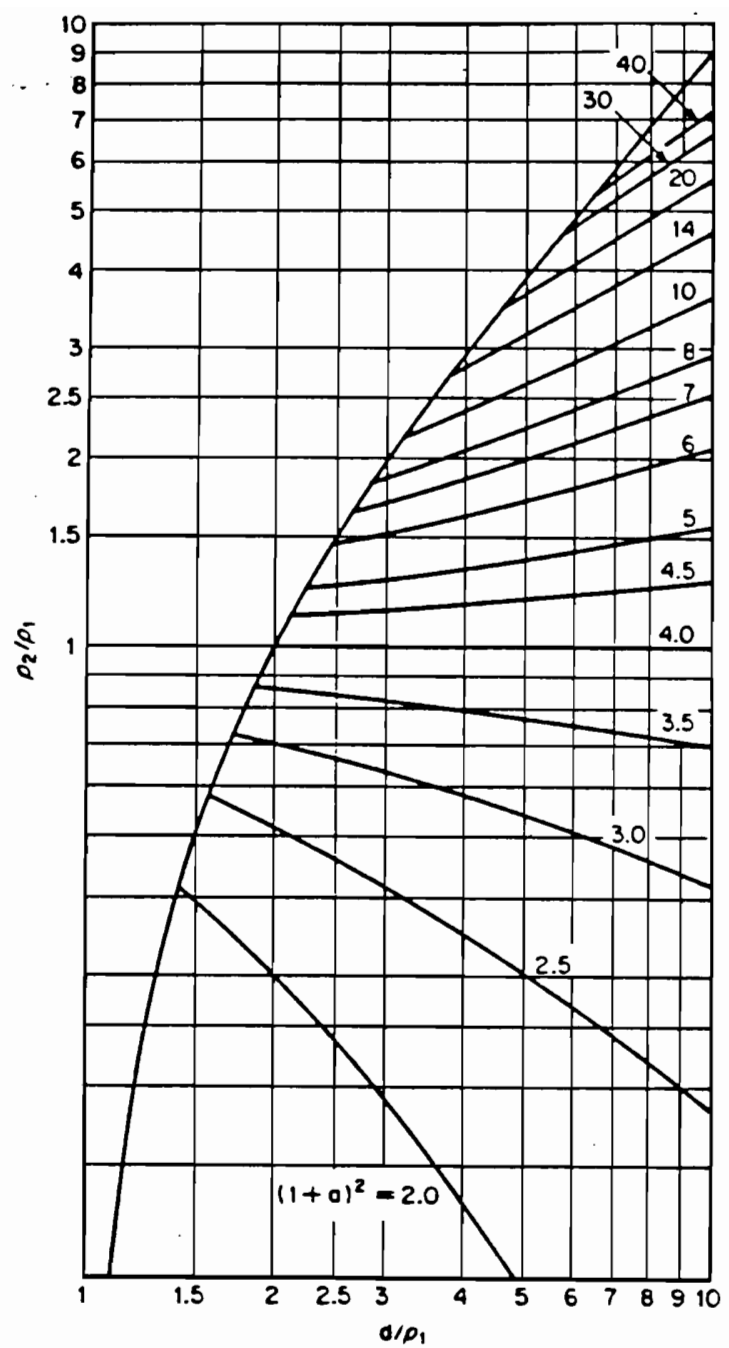

FIGURE 4.12: DIAMETER RATIO VERSUS IMPEDANCE TRANSFORMATION RATIO AND $d / P_{1} \cdot[6]$ 
A step-up ratio of 4.0 means that the antenna will have four times the impedance of an open half-wavelength dipole. We know this resistance to be about 72 ohms at resonance. Therefore, our folded dipole, constructed of two arms of similar diameter, should have a resonant resistance of $4 * 72=288$ ohms. As will be seen in the next section, this theoretical value is obtained with MININEC for all values of the wire separation $d$.

In practical thin-wire antenna geometries, the separation d between the conductors will be much larger than the wire diameter. The curves of figs. 4.12 and 4.13 are useful for design of thin wire folded dipoles for a relatively large radius/separation ratio. In effect, only for ratios close to the maximum absissa value of 10.0 , would these curves be useful.

In general, figures 4.12 and 4.13 show that folded dipoles give the designer a great flexiblilty, since almost any desired value of impedance can be obtained, from a few ohms to several thousand ohms, with adequate choise of wire radil and separation.

\subsubsection{MODELLING A FOLDED DIPOLE WITH MININEC}

It was necessary to model a conventional folded monopole using MININEC, to ascertain the validity of the program for this particular antenna. The data obtained was then compared to the theoretical model described in the previous section. This was considered as a requirement prior to the development of a valid model for the reduced-size folded dipole of chapter 5.0 .

Specifically, the resonant resistance, bandwidth and radiation pattern of a folded dipole were obtained with MININEC. Then, the most important feature of the impedance step-up ratio was analysed as obtained with the program.

First, the folded dipole of figure 4.14 was analysed. The wire separation is $1.0 \mathrm{~cm}$. and the wire radius (equal for both arms) was $0.1 \mathrm{~cm}$.

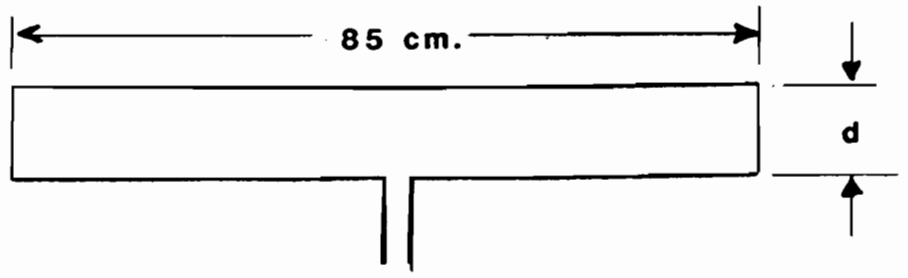

FIGURE 4.14: GEOMETRY OF FOLDED DIPOLE. 
The segmentation density was chosen to be 34 segments. This segmentation meets the criteria for segment length described in chapter 3.0 and provides adequate accuracy of the results.

The radiation resistance was found to be exactly 288.3 ohms for a resonant resistance of $170.2 \mathrm{MHz}$. This correlates very well with the theoretical resistance presented in the previous section.

EFFECT OF WIRE SEPARATION IN RADIATION RESISTANCE:

The separation of the two arms was changed for three cases. The results are shown in table 4.5 below.

TABLE 4.5: EFFECT OF WIRE SEPARATION ON FOLDED DIPOLE RESONANT RESISTANCE.

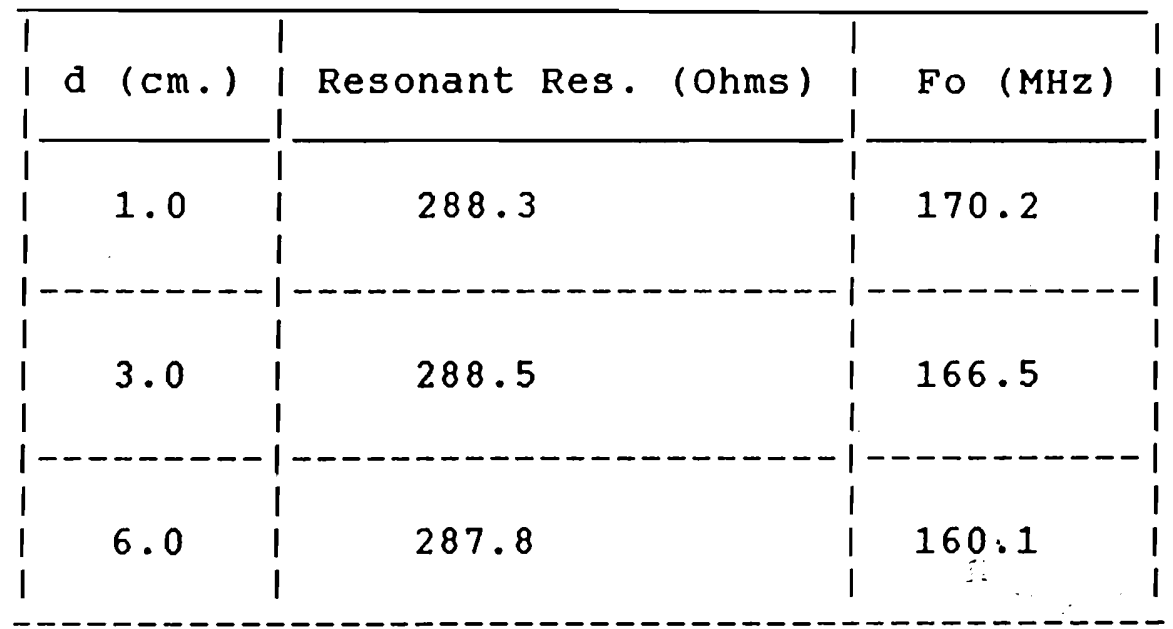

The radiation resistance remains practically the same as the wire separation $d$ is increased. This confirms the theoretical behaviour of folded dipoles.

On the other hand, it can be seen that the resonant frequency decreases as the wire separation is increased. In general, it can be sald that for values of d that are very small compared to half the wavelength, the resonant frequency is that of a half-wavelength dipole. It has already been shown in previous sections, how the resonant length of a dipole is somewhat shorter than half wavelength by a typical factor of 2 to 10\%. For the case where the folded dipole of length $85 \mathrm{~cm}$. has a wire separation $d=1.0 \mathrm{~cm}$. the resonant length is about 0.97 times the half-wavelength.

For the case where $d=6.0 \mathrm{~cm}$. the resonant length is almost 0.90 times half the wavelength. That is $10 \%$ shorter than half-wavelength.

The fact that an increase in wire separation changes the resonant frequency, is of course to be expected. When d is changed, so is the total length of the wire composing the antenna. The overall geometry is changed, and the distribution of electric and magnetic flelds are also changed. 
EFFECT OF WIRE SEPARATION IN ANTENNA BANDWIDTH

For the three values of d described previously, a frequency sweep was done with MININEC, and a set of impedances was obtained in each case.

In order to determine the antenna bandwidth, a method similar to the one employed for meander antennas in section 4.2 was used. The impedances were now normalized to a transmission-line value of 300 ohms. This was chosen since the 300 ohm parallel-wire line is a common, commercially avallable transmission line, that is the most often-used means of feeding folded dipole receiving antennas.

The normalized impedances were entered in a smith-Chart and a VSWR circle of 2.0 was used to determine the bandwidth.

The results are shown in the following table:

TABLE 4.6: BANDFIDTH OF A FOLDED DIPOLE AS FIRE SEPARATION d IS INCREASED

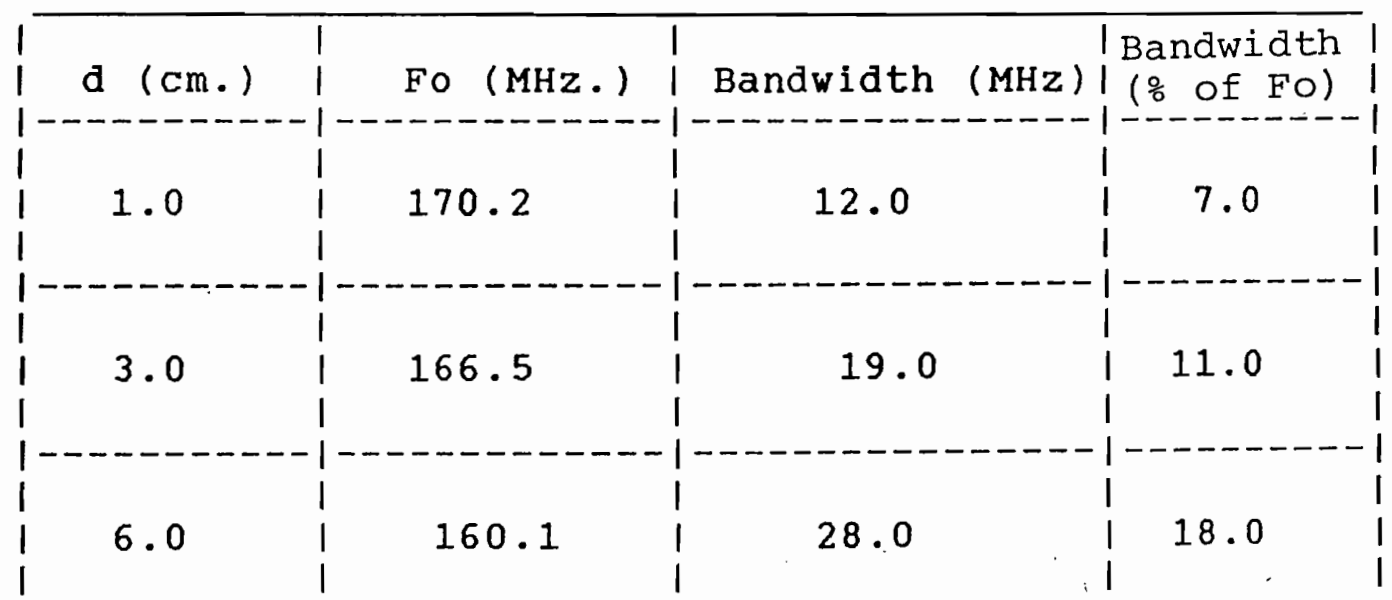

As the results show, the bandwidth is increased as the wire separation is increased. In this case, our study was concentrated on cases where the separation d is less than one-tenth of the length of the dipole. This is generally the case for folded dipoles, as it is desirable for structural reasons when the antenna is composed of a folded wire. 
The impedance values obtalned with MININEC, for folded dipoles with $d=1.0$ and $d=6.0 \mathrm{~cm}$. are shown in tables 4.7 and 4.8 . These correspond to the Smith-charts of figures 4.15 and 4.16 . Note that the Impedance behaves very much like that of a conventinal dipole: it is capacitive for frequencles below resonance, and becomes inductive past resonance.

Only the impedance obtalned with MININEC is given in these tables. It has already been sald that these impedances were normalized to 300 ohms to be entered in the smith-Charts. Also, note the value of the impedance near resonance at $170 \mathrm{MHz}$.

The values have been rounded to the nearest integer, for simplicity.

TABLE 4.7: IMPEDANCE OF A FOLDED DIPOLE WITH $\mathrm{d}=1.0 \mathrm{~cm}$.

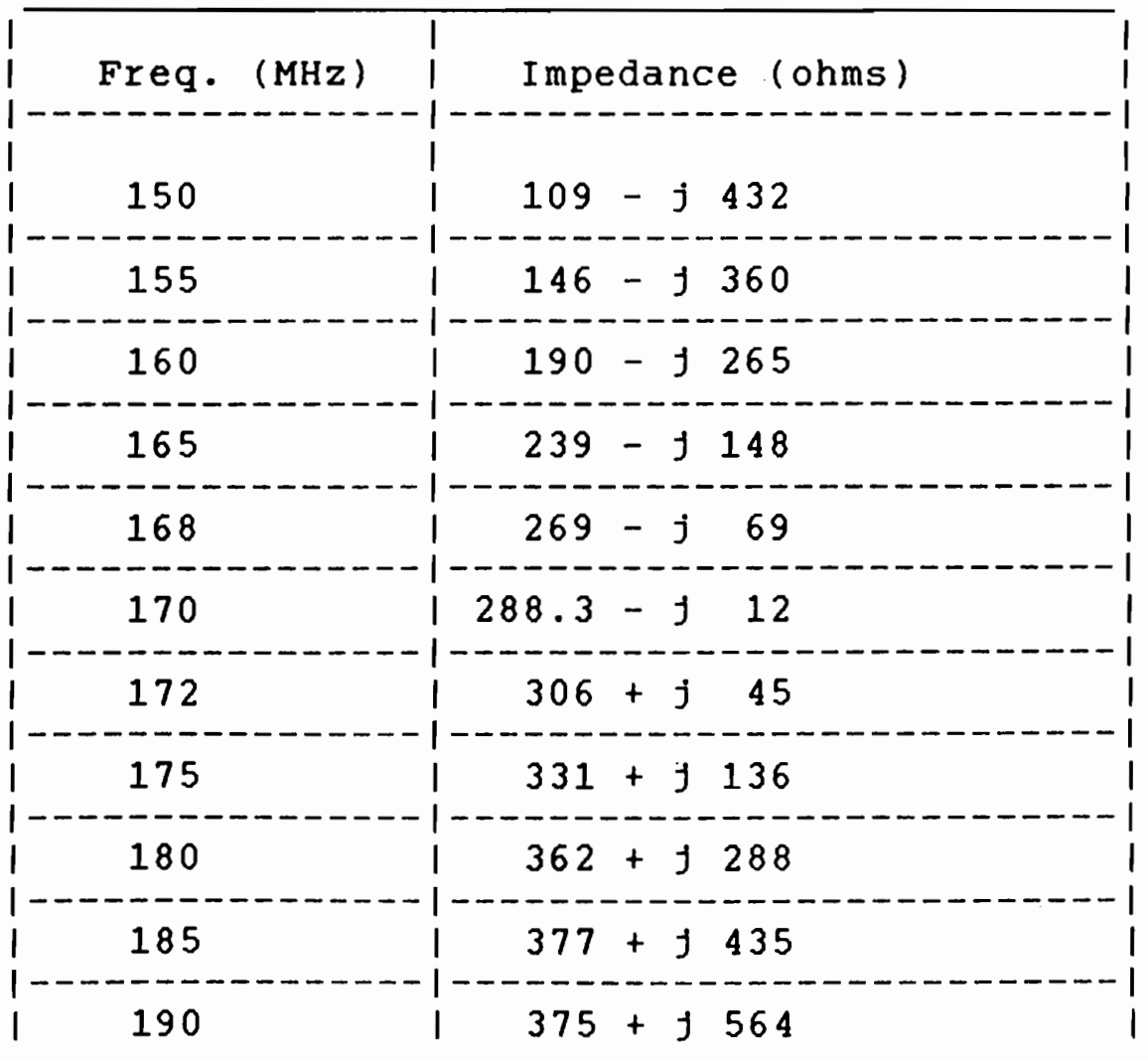




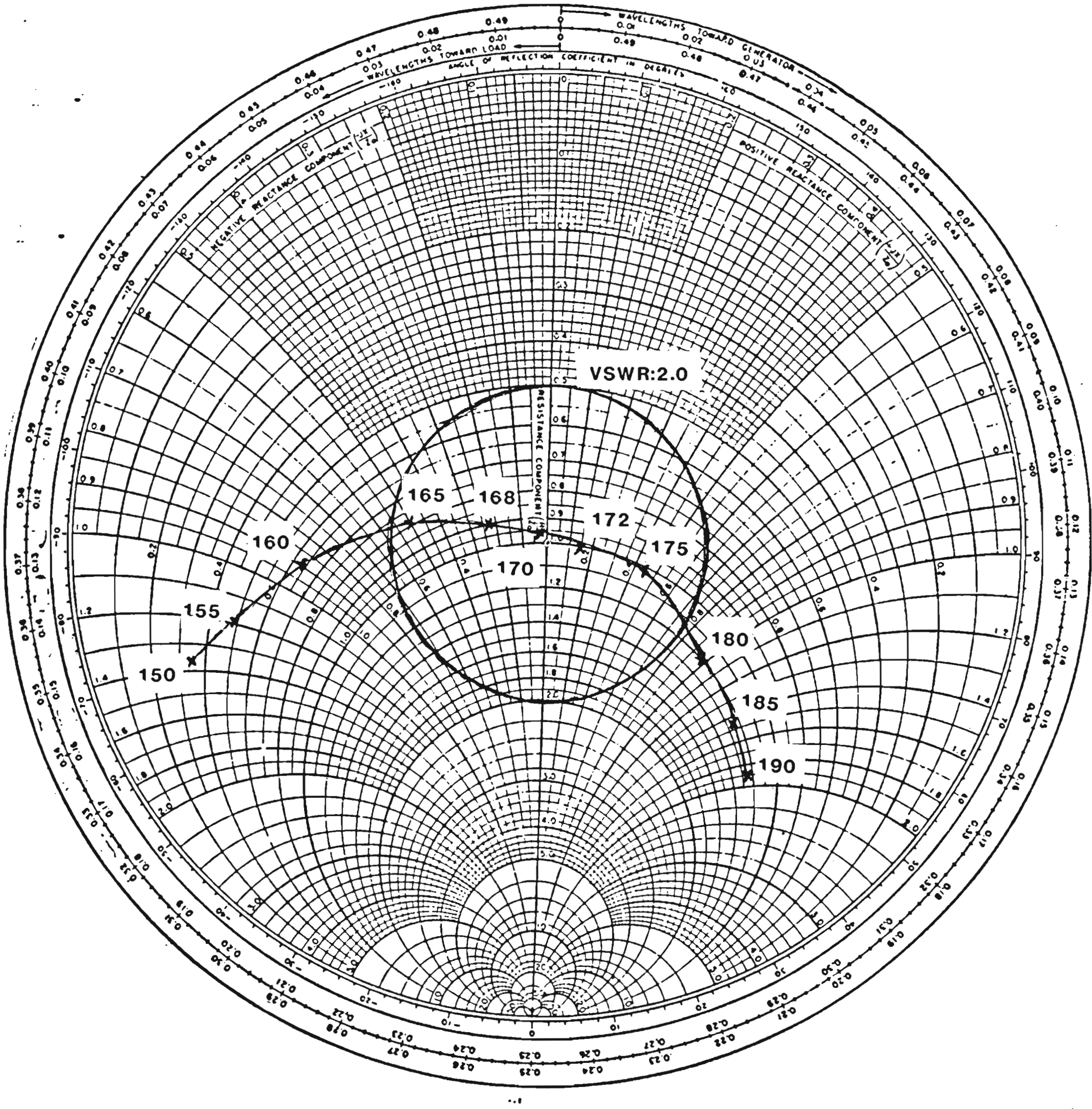

FIGURE 4.15 IMPEDANCE OF A FOLDED DIPOLE d: $1.0 \mathrm{~cm}$. 


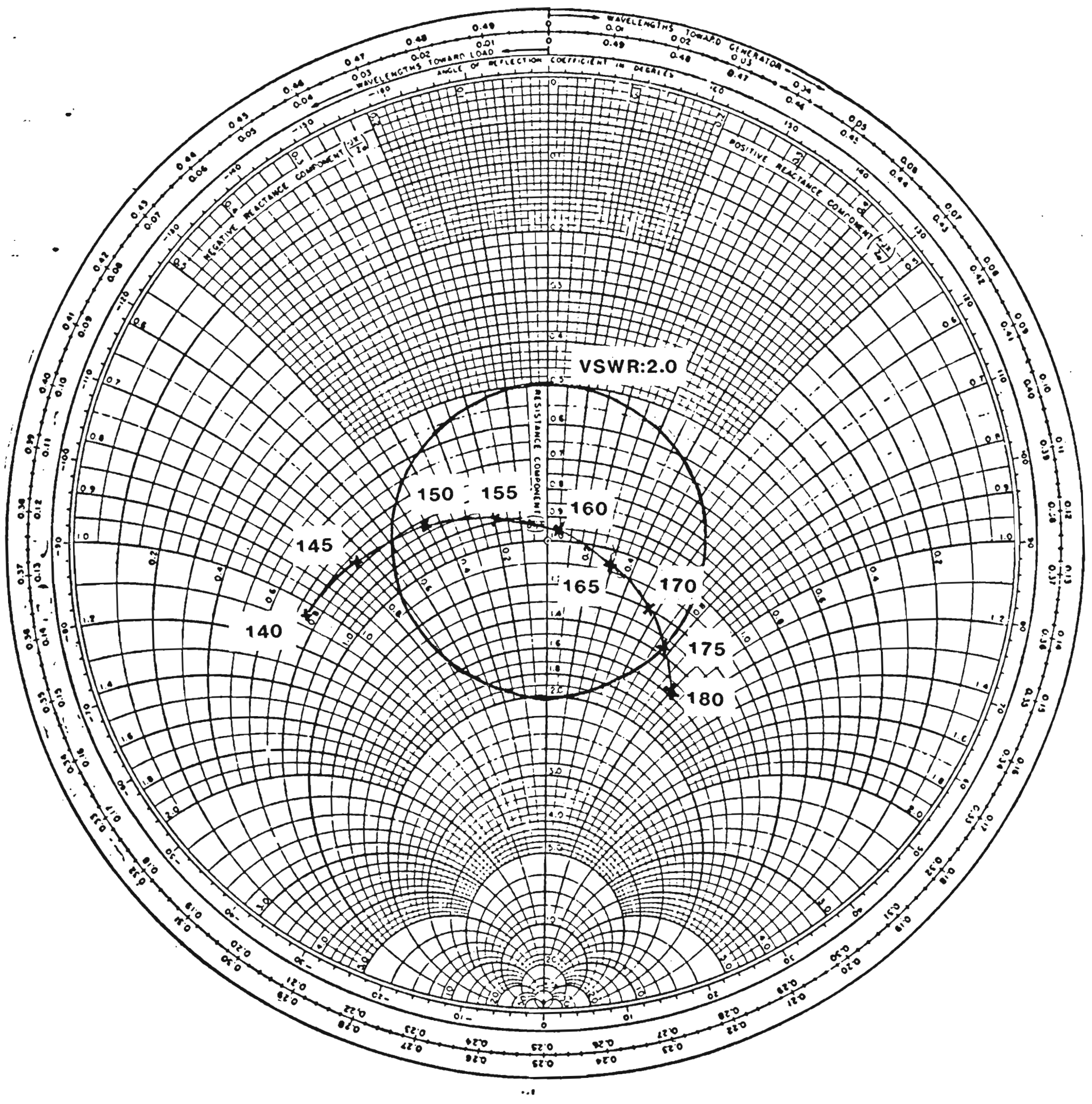

FIGURE 4.16 IMPEDANCE OF A FOLDED DIPOLE d: $6.0 \mathrm{~cm}$.

NORMALIZED TO 300 OHMS 
TABLE 4.8: IMPEDANCE OF A FOLDED DIPOLE WITH $\mathrm{d}=6.0 \mathrm{~cm}$.

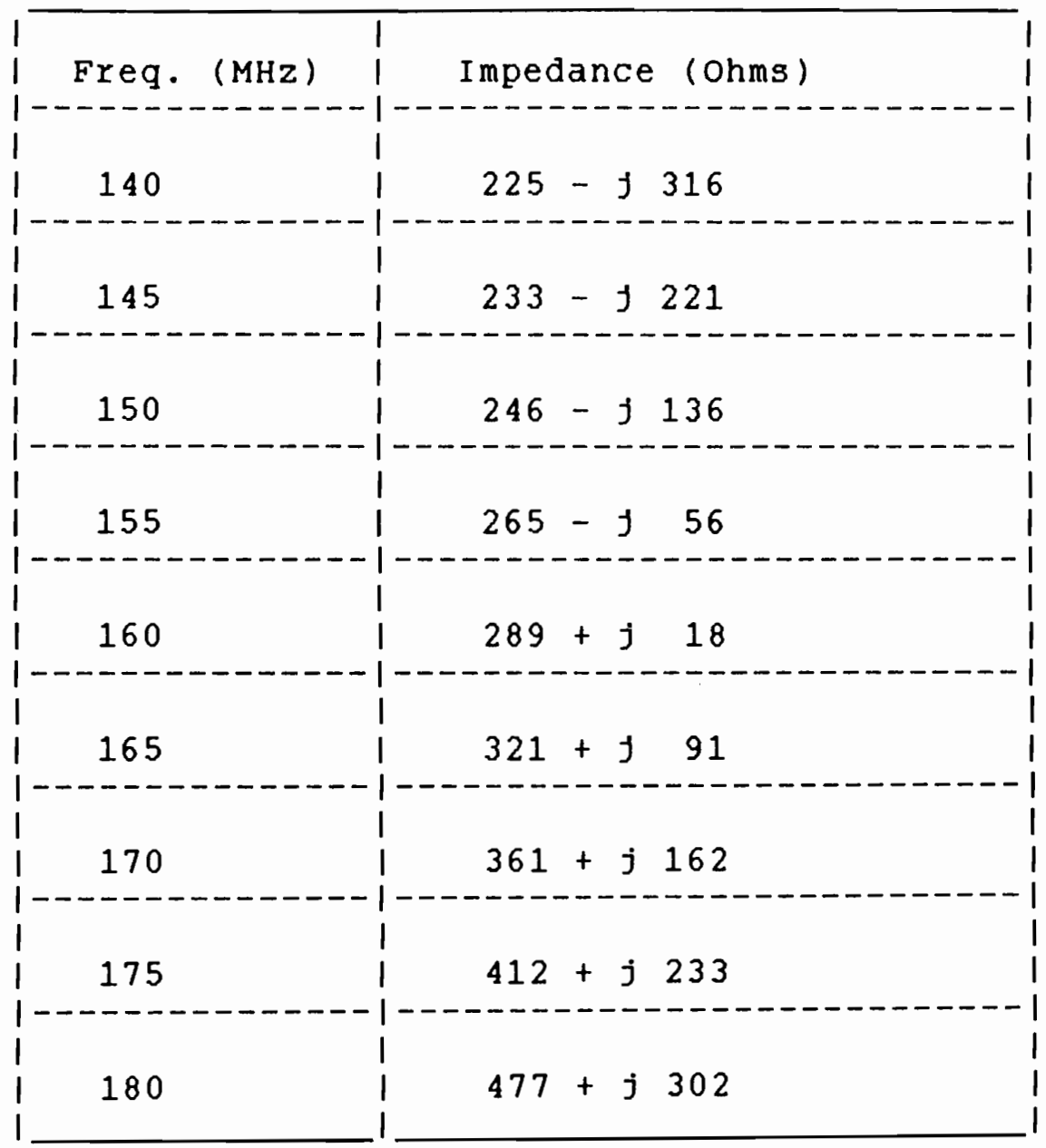

The radiation pattern of the folded dipole with d $=6.0 \mathrm{~cm}$. has been plotted by MININEC. It should be explained that the antenna geometry that served as the basis for this pattern, had both arms parallel to the $Z$ axis (vertical axis, in MININEC). The radiation pattern shown in figure 4.17 is that corresponding to the vertical pattern. Note that the gain is close to $2 \mathrm{dBi}$ and that the pattern corresponds to the well-known shape for a folded dipole. 
The problem of a stepped-up wire radius has been analysed with MININEC has been studied by the authors of MININEC (2). However, the model used was one of a single wire, with different wire radius steps. Furthermore, their model was only for an increase in wire radius with respect to the excitation point. The case of stepped-up radius in more complicated geometries was not analysed, nor was the the case of a step-down in wire radius.

Their results are quite encouraging, since MININEC follows very closely the theoretical and practical data it was compared to. The current distribution of a straight-wire antenna in free space is compared to results obtained with NEC and theoretical data.

In addition, the authors recommend that further analysis be conducted for more complex geometries, and for the case of a step-down in wire radius.

For the case of a folded monopole, it was necessary to establish the fact that MININEC would reflect the properties described in previous section, for the impedance transformation ratio.

The antenna described in figure 4.14 was modifled to change the diameter of the second arm. This is the arm opposite the one that is being excited.

First, the folded dipole with $d=1.0 \mathrm{~cm}$. was modified for several radius changes in the second arm, then the impedance transformation ratio was calculated and compared to that of figure 4.12 .

The reason for choosing the value of $d=1.0 \mathrm{~cm}$. was that the curves of figure 4.12 and 4.13 are drawn for a maximum value of d equal to ten times the radius of the excitation arm of the dipole. Our dipole of Eigure 4.14 has a wire radius of $0.1 \mathrm{~cm}$, therefore our $\rho_{1} / d$ ratio would be equal to 10 in this case.

The results of this analysis are shown in the table below. It can be seen that for stepped-down radius, the results differ signiflcantly from the theoretical values of the impedance transformation ratio.

For values steps in radius greater than 3.0 the results also show a marked divergence from the reference data.

The reason behind these discrepancies can probably be found in the low value of d when compared to the wire radius. In effect, the stepped-up radius (greater than $0.3 \mathrm{~cm}$ in the worst cases) is a significant percentage of the wire separation. 
FIGURE 4.17: VERTICAL RADIATION PATTERN

FOLDED DIPOLE

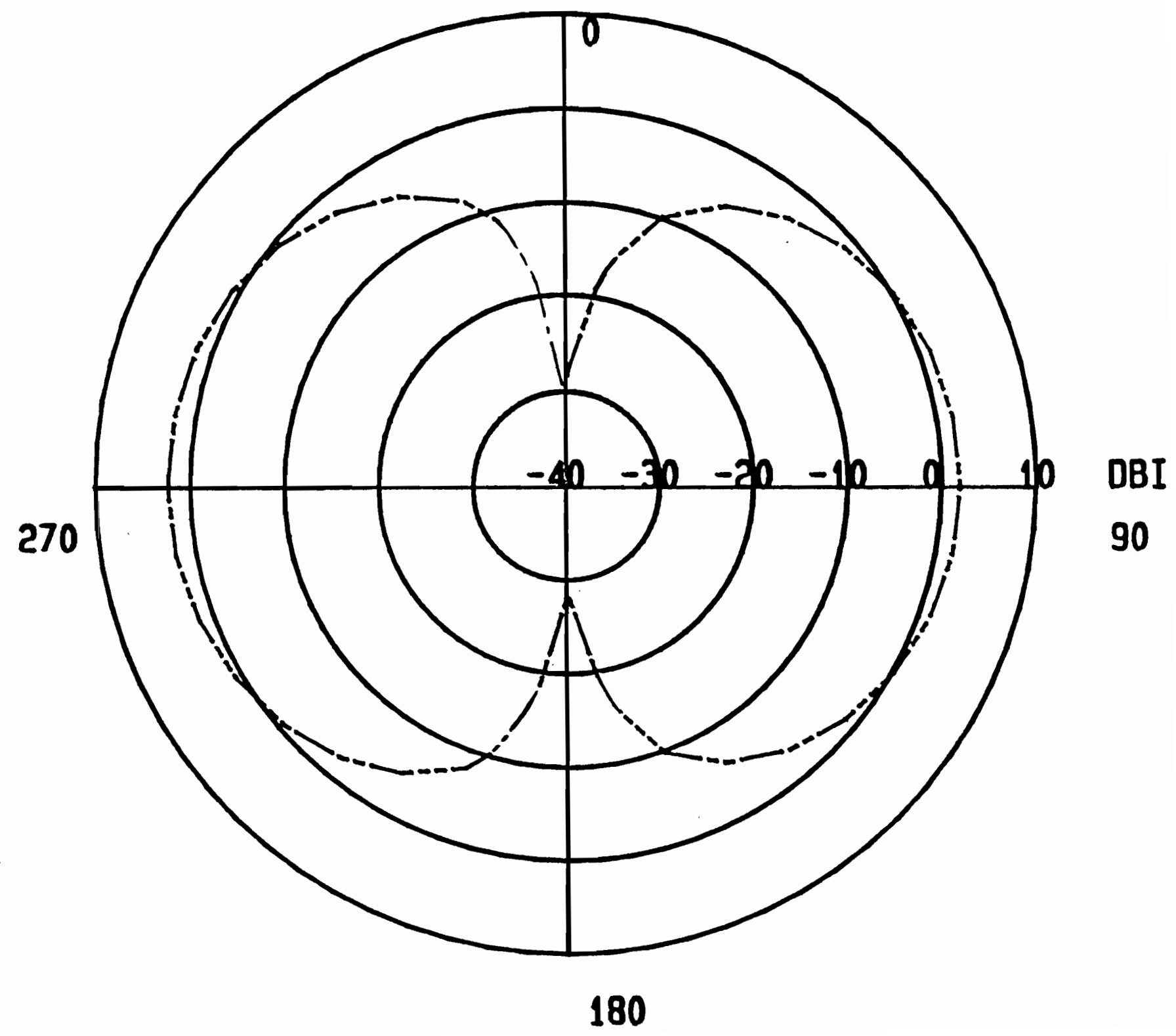


TABLE 4.9: IMPEDANCE TRANSFORMATION RATIO OF A FOLDED DIPOLE WITH $\mathrm{d}=1.0 \mathrm{~cm}$.

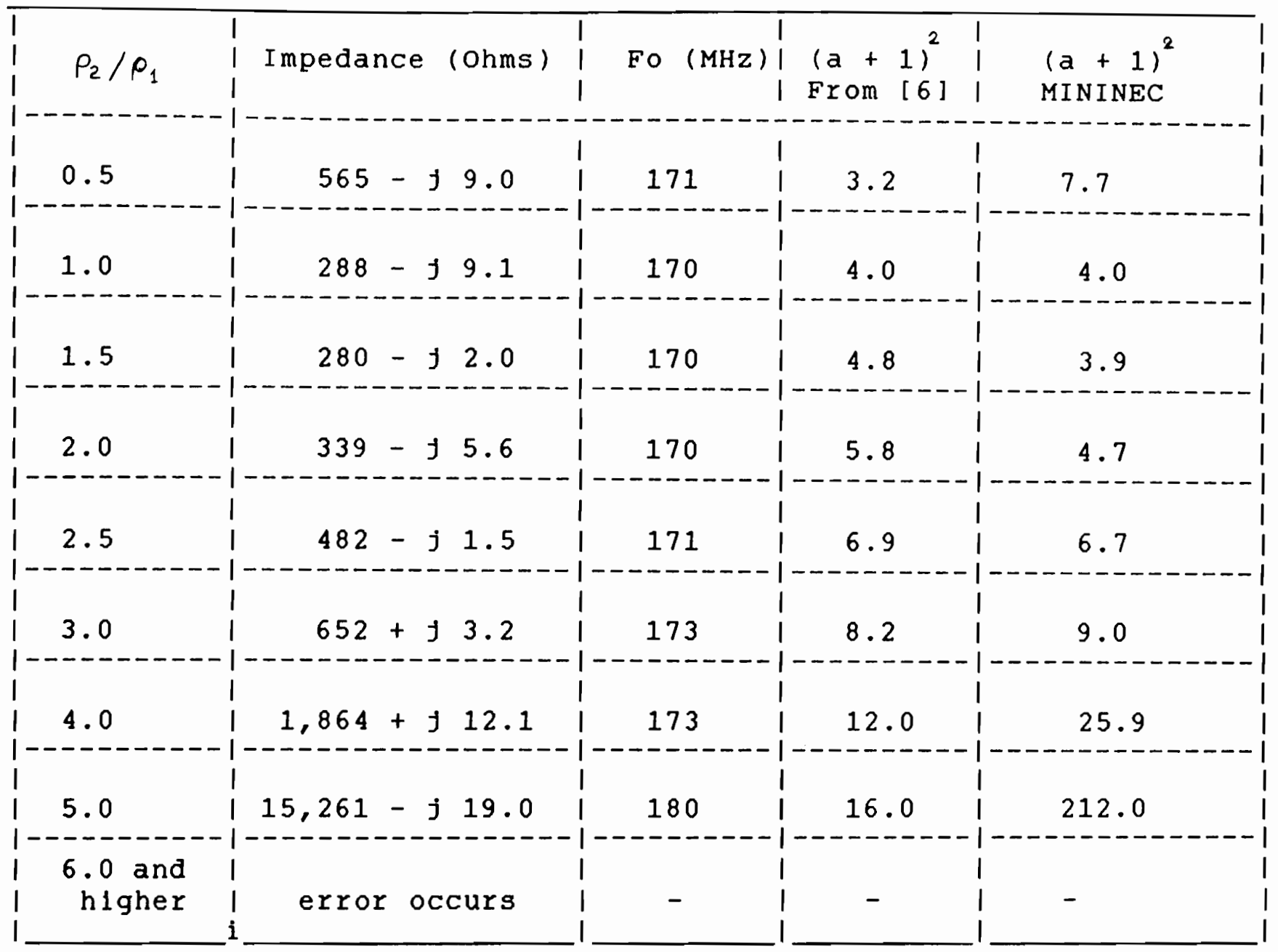

It is also worth noting that the resonant frequency is also changed as the ratio of the radii is increased.

This again, is due to the fact that the antenna geometry is modifled and therefore, so is the electric and magnetic fields spacial distribution.

The program, however, still gives acceptable values of impedance for radii ratios of less than 3.0. The exception, of course is the case where this ratio is 1.5 . But for the rest of the values shown in the table, below 3.0 , the program still seems to follow the theoretical data, within acceptable limits. 
The next step was to try a different geometry. The obvious cholse was to increase the wire separation d. As was previously pointed out, it was suspected that for wire radi1 that are comparable in size with the wire separation d, the geometry of the antenna seems to be excessively changed to give accurate results.

Therefore, the separation $d$ was changed to $d=6.0 \mathrm{~cm}$. and the antenna impedance was calculated again with MININEC, for several different radi1.

The results, as shown in the following table, are far more acceptable than the previous.

TABLE 4.10: IMPEDANCE TRANSFORMATION RATIO OF A FOLDED DIPOLE WITH $\mathrm{d}=6.0 \mathrm{~cm}$.

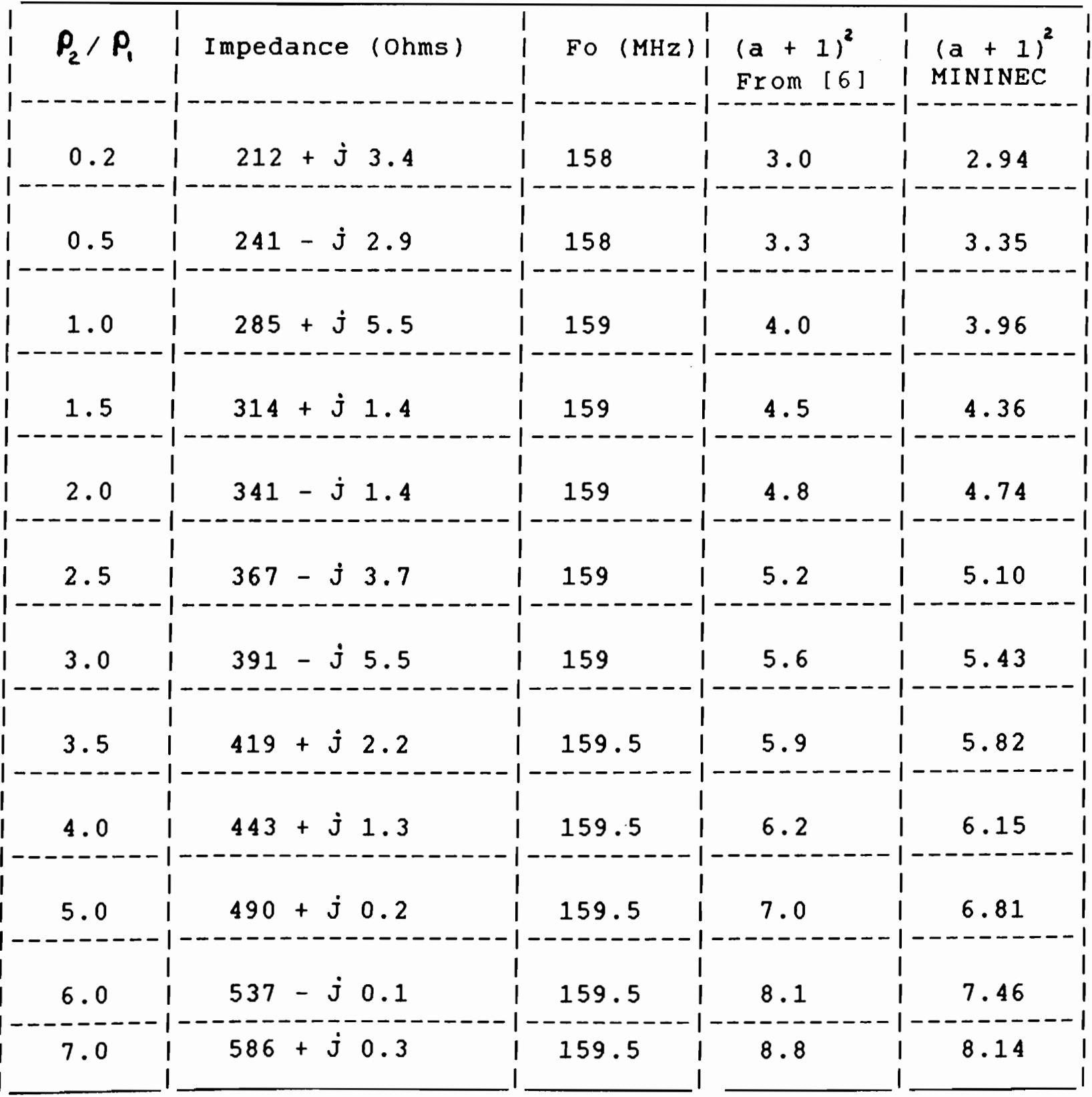




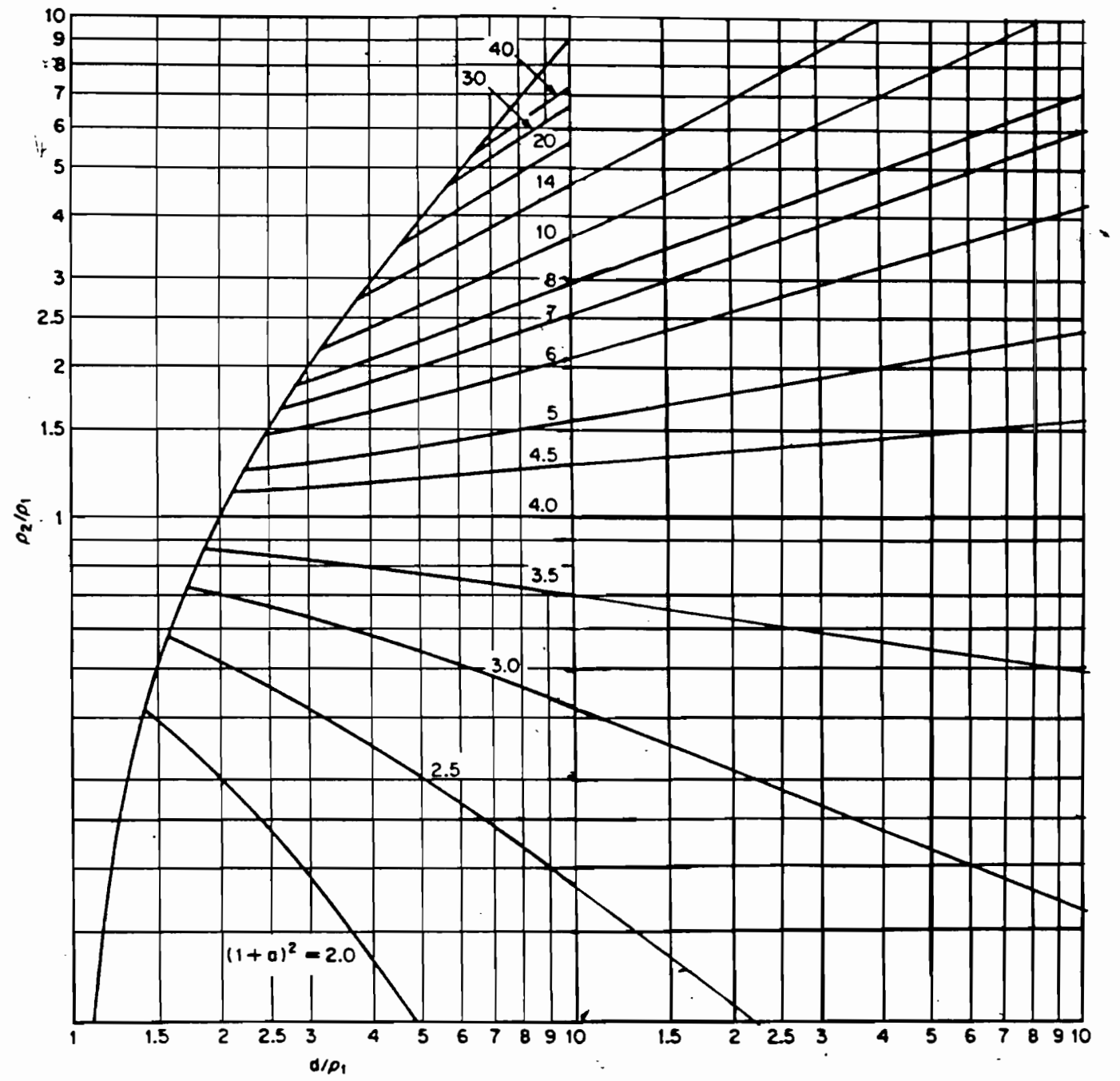

FIGURE 4.18 IMPEDANCE TRANSFORMATION RATHO

EXTRAPOLATED FROM FIGURE $\mathbf{4 . 1 2}$ 


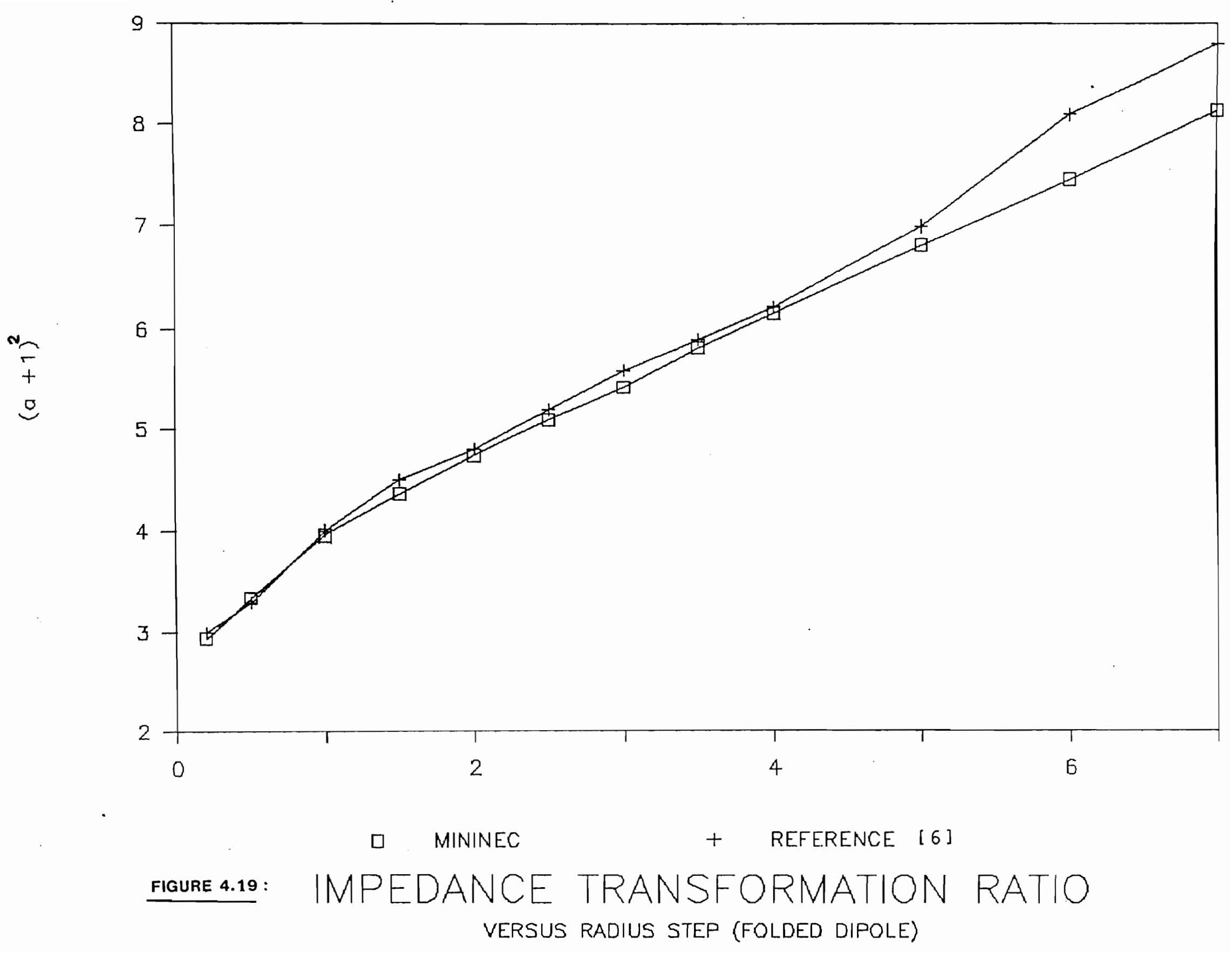


The reference values for the impedance transformation ratio were not readily avallable from the graphs of figures 4.12 and 4.13 . These curves only cover a maximum $d / p_{1}$ ratio of 10.0 . To obtain the required ratio for the antenna being analysed, that presented a wire-radius to separation ratio of 60.0 , an extrapolation of the graph of figure 4.12 was done.

Basically, the logarithmic scale of the abcissa was extended, to obtain the required value of 60.0 for the radius-to-separation ratio, and the curves for each value of the impedance transformation $(a+1)$ were extended using straight lines. This, of course lends itself to some degree of error, but the objective of the analysis with MININEC was only to obtain some degree of confidence in modelling folded dipoles, with stepped wire radius.

The resultant set of curves is shown in figure 4.18

Also, some of the reference values are, of course approximations at best, due to the low resolution attainable with this extrapolated set of curves.

Being that as it may, the MININEC results are amazingly close to the ones used as reference. This is a big contrast with the results obtained for the folded dipole with $\mathrm{d}=1.0 \mathrm{~cm}$.

It can be seen that for almost the full range of radil steps, the impedance transformation ratio is very close to the reference value. For larger steps, greater than 5.0, the difference between MININEC and the reference seems to increase. But, in observing figure 4.18, it can be seen that the graph resolution is less for higher values of the step, due to the nature of the logarithmic scale.

The differences therefore, can be due to errors or differences in the extrapolated curves, with the practical values.

Figure 4.19 shows the two sets of values for the impedance transformation ratio. The MININEC values show some divergence from the reference values, for higher values of the abcissa. 
An important consideration about the folded dipole, as an antenna geometry, is that its is basically a wire-loop. In that respect, the folded dipole impedance is very much like that of a loop antenna. For very low frequencies (or as the operating frequency approaches zero, or DC) the impedance is basically a short circuit. As the frequency is increased, the loop or folded dipole behaves like one-turn coil, and the impedance becomes more inductive.

Now, we have seen in Figures 4.15 and 4.16 that the folded dipole is resonant for lengths of about half-wavelength. Furthermore, the impedance is capacitive for frequencles below resonance and becomes inductive for frequencies above resonance. This seems to be in disagreement with the impedance behaviour of loop antennas.

The discrepancy can be explained by the fact that what we call "resonance", is not in fact the 1st point of resonance, as is the case of most antennas (e.g. dipoles and monopoles). The folded dipole is always operated at frequencies around the second resonance point, sometimes called anti-resonance.

Resonance is understood here as any point where the antenna reactance becomes equal to zero, with the resistance being a finite number.

The following flgure shows a typical folded-dipole impedance curve.

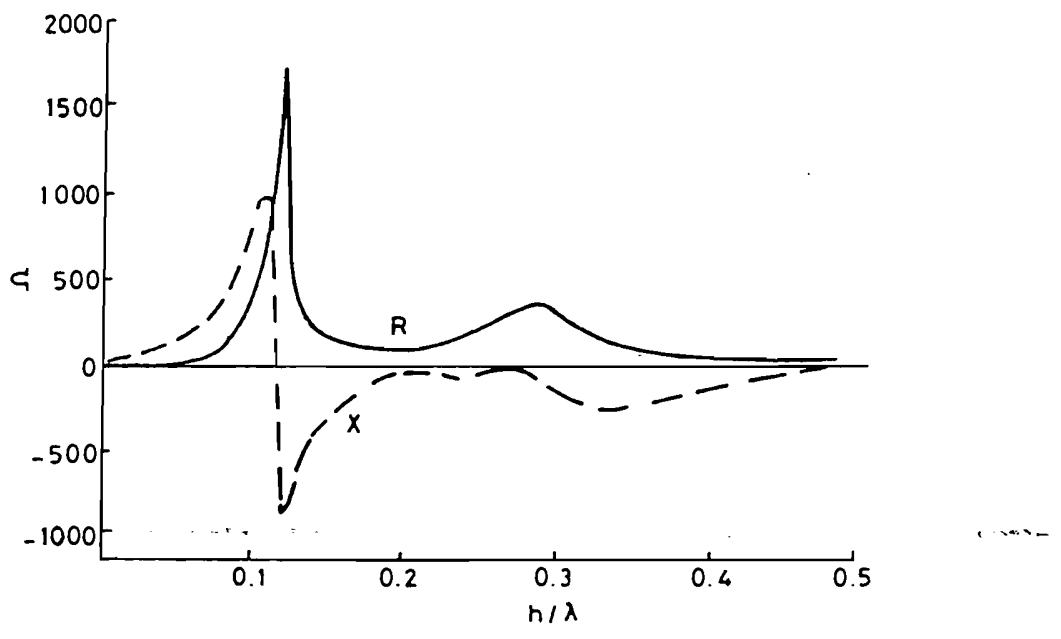

FIGURE 4.20 - IMPEDANCE OF A FOLDED DIPOLE [9] 

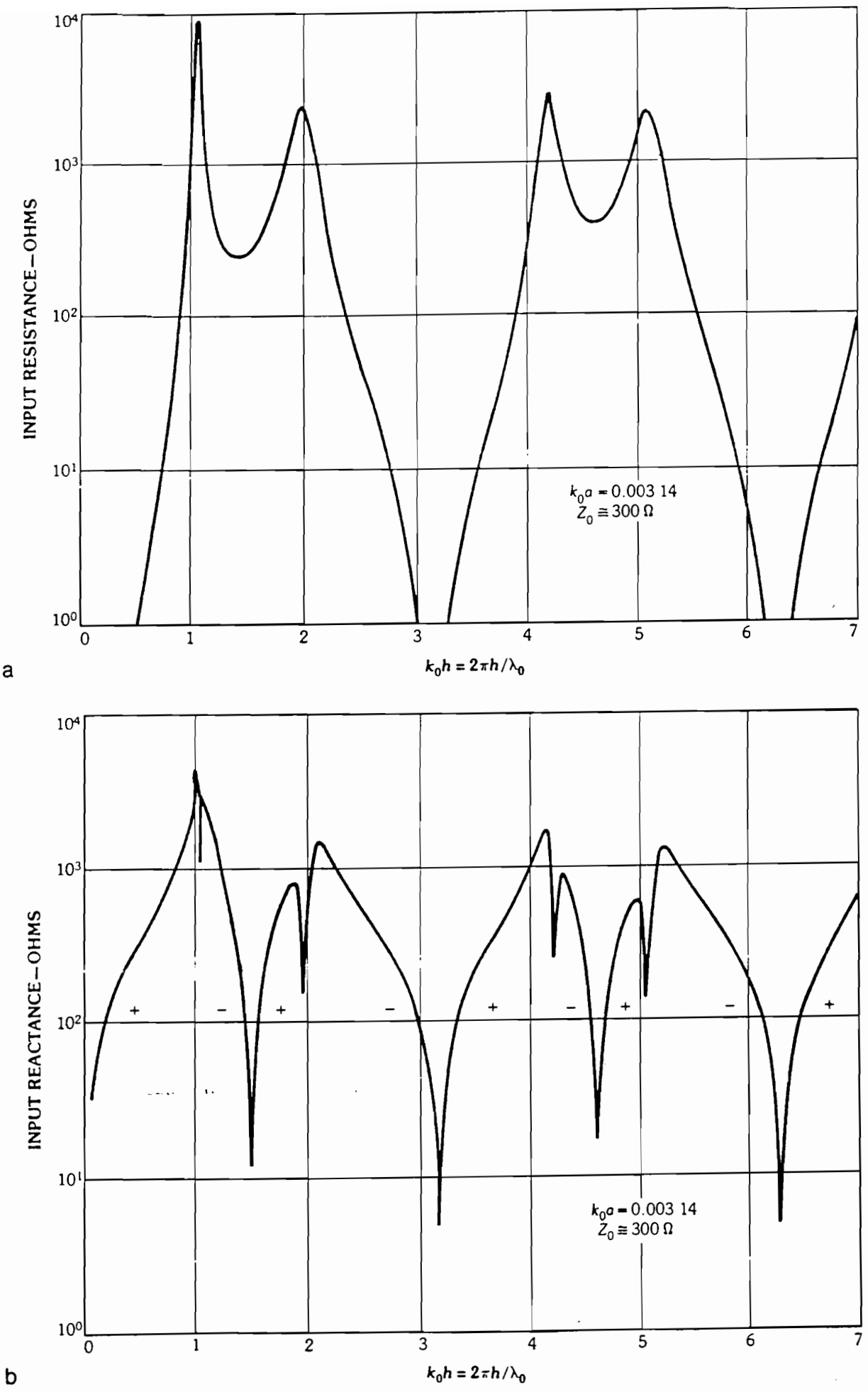

koa : Electrical radius of both arms

FI IGURE 4.21: INPUT IMPEDANCE OF A FOLDED DIPOLE, AS A FUNCTION OF THE ELECTRICAL HALF-LENGTH $k_{0} h$, WHERE $h=L / 2$ (radians) [9] a) RESISTANCE b) REACTANCE. 
The second resonance, or normal operating frequency of the folded dipole occurs in the curves at the first "valley", where the resistance reaches the known value of 300 ohms.

This point of second resonance occurs at a half-length of aproximately 1.6 radians. This corresponds to a length of around a half wavelength.

It can be seen that the reactance also shows a sudden inversion, indicating a crossing of the zero-reactance value. This inversion, however is not as sharp as the one for first resonance, thereby allowing for the operating bandwidths described in the previous section.

\subsubsection{MODELLING FIRST RESONANCE WITH MININEC}

Our last step in analysing the folded dipole antenna characteristics is to find out how MININEC will model the first-resonance impedance values for this geometry.

The antenna of section 4.4 .3 was used for this test. The separation of the wires was $d=6.0 \mathrm{~cm}$. and the wire radlus was the same for both arms at a value of $0.1 \mathrm{~cm}$.

Again, the antenna was composed of 4 wires, totalling 34 segments. The results from MININEC show very good correlation with the data presented in the previous section.

Naturally, the ohmic values of impedance vary greatly from those of figure 4.21. This is of course due to the fact that our antenna has a separation-to-radius ratio of 60.0 , and not 12.5 as is the case of the reference figure.

The results are shown in table 4.11, and also in graphlc form in figures 4.22 (Resistance) and 4.23 (Reactance)

It can be seen that first resonance occurs at a frequency between $94 \mathrm{MHz}$ and $95 \mathrm{MHz}$, whereas second resonance happens at the expected frequency of $160 \mathrm{MHz}$.

At $94 \mathrm{MHz}$, the antenna has a length of about 0.27 of the wavelength, which is in accordance with the predicted value of approximately 0.3 wavelengths for the first resonance.

In figure 4.22, the upper limit of the graph has been set to 2000 ohms, in order to appreciate im more detail the lesser values of input resistance. However, from the table, we can see that the resistance value exceeds by far this upper limit (almost $20 \mathrm{Kohm}$ at 95 Mhz.)

Also, flgure 4.23 (reactance) shows the very rapld change from positive to negative at little below $95 \mathrm{MHz}$. 
In the previous figure, the parameter $h$ is the antenna half-length. Therefore, the folded dipole operating point, would be around the abcissa value of 0.25 .

It can be clearly seen that first resonance occurs at apoint well below the half-wavelength point.

This first resonance is characterized by:

1) - A very high value of resistance.

2) - A very sudden change in reactance, from inductive to capacitive as frequency is increased. Also, the reactance values around resonance are very high, in the order of a thousand ohms.

These impedance characteristics of the first resonance is what make the use of a folded dipole impractical around this frequency. In particular, the high value of the resistance is a big impediment for practical uses. In addition, the very steep change in reactance makes the possible bandwidth of utilization very small, when compared to that of the second-resonance point.

Flgures $4.21 \mathrm{a}$ and $4.21 \mathrm{~b}$ show a more detalled view of the 1mpedance curve of a folded dipole (9). The abcissa gives the value of the electrical half-wavelength, expressed in radians.

The curves correspond to a particular dipole, where the radil a of both conductors is the same, and ko* $a=0.001 * \pi$, and their separation remains the same at $d=12.5 * a$, thereby yielding a nominal transmission line impedance of $\mathrm{Z} O=300 \mathrm{ohms}$.

Naturally, the actual values of the impedances and frequencles involved, will vary with the wire separation and the raddi of the two antenna arms.

It can be seen from the set of curves of flgure 4.21 that the first resonance occurs at a value of the electrical half-wavelength of around 1.1 radians. This corresponds to an antenna length of about 0.3 wavelengths.

The resistance at this point exhibits a sharp peak in value. The maximum of this peak is in the order of several thousand ohms.

The reactance, on the contrary, shows a sharp inversion point. The impedance below resonance is inductive and increases very rapldiy; The actual inversion in reactance is very sharp and is seen in the curves only as a vertical line. Both the inductive and capacitive values of the Impedance above and below resonance are of the order of several thousand ohms. 
TABLE 4.11: FIRST RESONANCE OF A FOLDED DIPOLE.

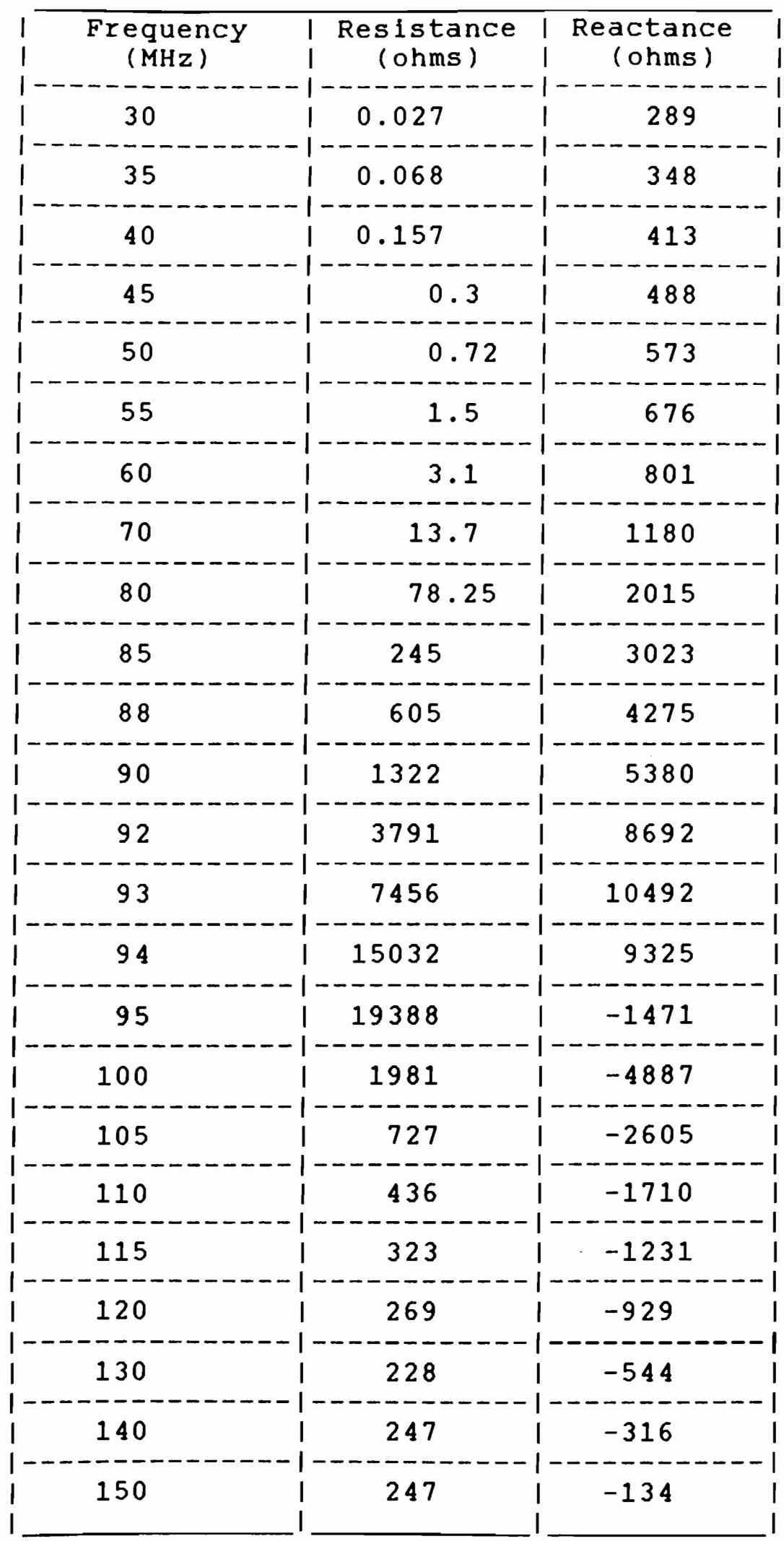




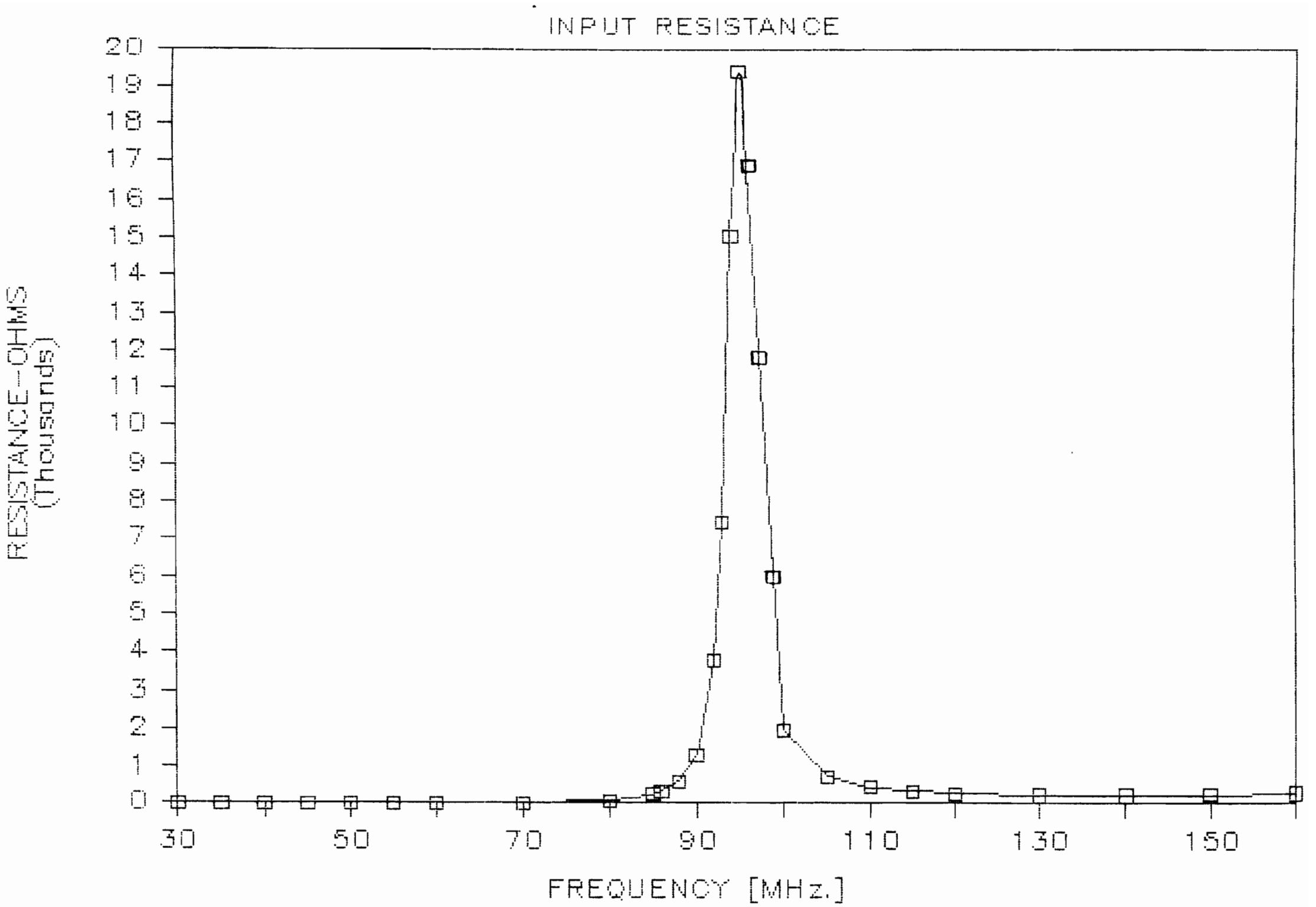

FIGURE 4.22: INPUT RESISTANCE OF A FOLDED DIPOLE, L $=60 \mathrm{~cm}$. AND D $=6 \mathrm{~cm}$. COMPUTED WITH MININEC. 


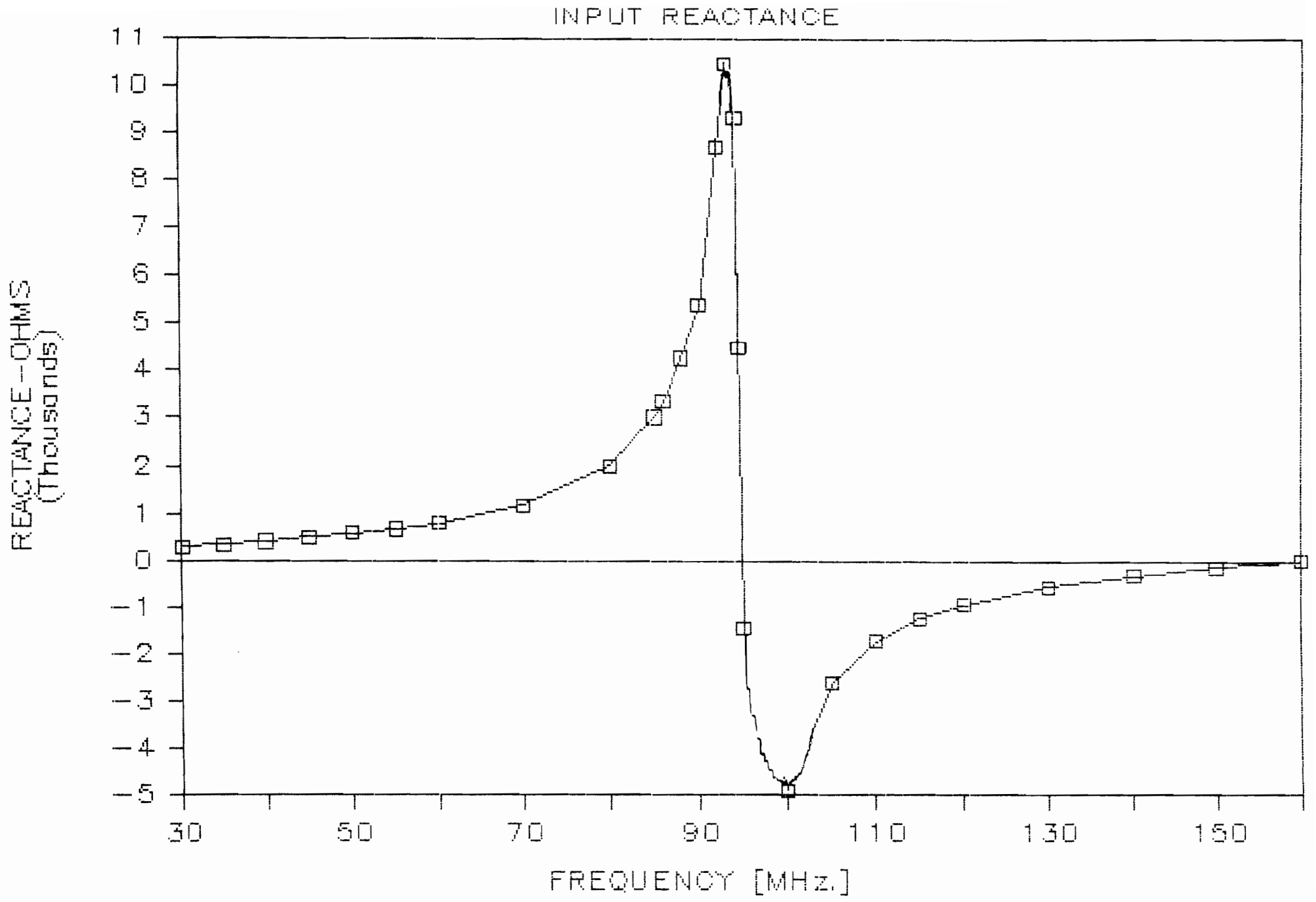

FIGURE 4.23: INPUT REACTANCE OF FOLDED DIPOLE, L $=60 \mathrm{~cm}$. AND D $=6 \mathrm{~cm}$. COMPUTED WITH MININEC 
Note how the reactance tends again to zero as the erequency approaches second resonance. However, the slope of this curve is a lot smaller than the sharp change encountered for first resonance. This makes for a moderate bandwidth around second resonance.

\subsection{CONCLUSIONS}

As a conclusion to this chapter, it can be sald that MININEC has been proven as a valid tool for modelling folded dipoles, and meander antennas, both of which are the basic components of the design described in this paper.

For the stepped radil problem, it is obvlous that only geometries where the wire separation is much larger than the excitation-wire radius, can be used with MININEC. From the experiment described in this section, it seems that a ratio of $\rho_{1} / d$ equal to 10.0 is still not large enough to be modelled with MININEC.

For practical geometries, however, where thin wires are used, $1 t$ is always possible to obtain ratios of the order of 50 and above.

For the case-study of the folded dipole impedance, MININEC has been proven to react very well for most of the frequency range of interest. However, one must keep in mind that most errors in MININEC are encountered as the frequency is increased, not decreased because the segment lengths tend to be too big compared to the wavelength.

In general, enough data was gathered and geometries were studled as explained in this chapter, to gain sufficient confidence in the program. This allows to treat the MININEC results obtalned while designing the reduced-size folded dipole as accurate, within the constraints of the program. 
5.1 DESIGN OBJECTIVE:

In this chapter, we will describe the design of the reducedsize folded dipole, using only the MININEC program.

The idea of designing a reduced-size folded dipole, was generated while studying the properties of meander antennas. These antennas have been described in previous sections and have been analysed using MININEC. Thus, we have already proved the validity of the program for the design of this type of geometry.

In studying the characteristics of meander antennas, as a means of reducing the size of dipoles and monopoles, it is evident that their low resonant resistance is the one characteristic that can make these antennas less atractive for practical applications. The fact that some sort of matching network or device must be used to adapt the input impedance of the antenna with the relatively higher values of impedance of commercial transmission lines, adds to the cost and the complexity of the antenna system, therefore making it less atractive for real-world applications.

The other characteristic that appears to be somewhat questionable is their narrow bandwidth. However, it is not always desirable to have a wide bandwidth. On the contrary, for some fixed-channel applications, it would be advantageous to have a small bandwidth. This property reduces the effect of adjacent channel interference and in general, adds to the selectivity of the system.

In studying the folded dipole, as covered in previous chapter, it is obvious that this type of antenna provides a means for the designer to choose the antenna input impedance from a wide range of values. This is accomplished, as was explained before, by means of varying the diameter of the wire in the second arm of the folded dipole, or by increasing the separation between arms, or by a combination of both.

of course, this impedance-adaptability seemed interesting enough to be investigated further and possibly, to be applied to meander antennas. Ideally the objective of the design was to obtain a reduced-size antenna that had an impedance that did not require any matching to common transmission lines.

of course the common values of commercially available transmission line impedances of 50,75 and 300 ohms were a valid goal. 
Naturally, other important antenna characteristics had to be maintained, or rather obtained, by the final geometry.

Some of this preliminary design specifications are:

1- Gain had to be comprable to that of a standard folded dipole. or about $2.4 \mathrm{dBi}$. This refers to the gain of the antenna proper, without any parasitic elements.

2- The antenna should be as general-purpose as possible. That is, we are interested in designing reduced size common antenna, fit to be used in a wide range of frequencies, with proper scaling of dimensions. It could be a transmitting or receiving antenna.

3- Radiation pattern should also be comparable to that of a dipole or folded dipole. That is, directivity should be moderate.

4- Needless to say, the final geometry, as designed with MININEC, had to be phisically feasable. For example, wire radius, bends, wire separations and other such dimensions had to be attainable in the process of constructing the antenna. Furthermore, wire radii should, if at all possible, reflect commercially available types of thin-wire.

5- The value of the resonance resistance had to be one of the following values of characteristic impedances for common transmission lines: 50,75 or 300 ohms. At least, the design should be such that the resonant impedance obtained should not have to be matched to the transmission line ( 50,75 or 300 ohm), for a VSWR of less than 3.0 .

6- Bandwidth should be as great as poosible.

These were preliminary design objectives, or specificifications. In addition, although antenna efficiency is not mentioned here as a design goal, it is a parameter that should always be kept in mind in any antenna design.

From previous studies ( 3 ), we know that efflciency of meander antennas is comparable to that of the conventional antenna. This fact gives the designer some reassurance, if not the certainty, that designs involving meander dipoles or monopoles are likely to have relatively good values of antenna efficiency.

The design of the subject reduced-size folded dipole with MININEC met many problems, specially due to the program's inherent limitations. These will be described in detail as we cover some of the most important aspects of this design. 
The meander dipole, will be analysed in this section. This analysis is important, since the meander dipole is the basic component of the folded meander dipole antenna.

The theoretical results obtained with MININEC for this geometry are valuable, since they will serve to understand better the particulars of the folded version. This analysis was not included as part of the preliminary data analysis of chapter 4.0 because there exists no reference data for single folded dipole antennas, to compare the MININEC results with.

However, the results described in chapter 4.2 , for meander monopoles are undoubtly valid for estimating the accuracy of the results for meander dipoles.

The analysis was done as a necessary preamble to the MININEC design of the final antenna, in order to give the designer some guidance on expected results and characteristics for the final configuration. These results, added to the data gathered previously for folded dipole geometries, were of unvaluable use in determining and modeling the folded meander dipole antenna.

In this case, only antennas with $\mathbf{N}=2$ were analysed $(\mathbf{N}=$ number of meander sections per wavelength). The reason for this was that, as was shown in chap. 4.2, antennas with lower $\mathbf{N}$ have a greater reduction in size. Of course, the impedance is also smaller for a lower $N$, but this was not considered to be a major drawback, since it was expected that the impedance-transformation properties of folded dipoles could be used to obtain an appropriate value of input impedance in the final design.

Figure 5.1 shows the geometry for a meander folded dipole with $N=2$. The length of the dipole is $60 \mathrm{~cm}$. and it was modelled with MININEC as having 45 segments. The dipole, of course, was center-fed with a voltage source of 1.0 volts. The segmentation chosen provides adequate accuracy of the results for all the frequencies involved.

The wire separation, w, was taken as a variable. Three values of $w$ were tried for the meander dipole with $N=2$, and the antenna was subject to the same tests as those performed for the meander monopoles.

The objective was to determine the effect of $w$ on antenna characteristics. We have concentrated our study in two important parameters: the resonant resistance and the antenna bandwidth, for a VSWR value of 2.0. The results are as follows: 


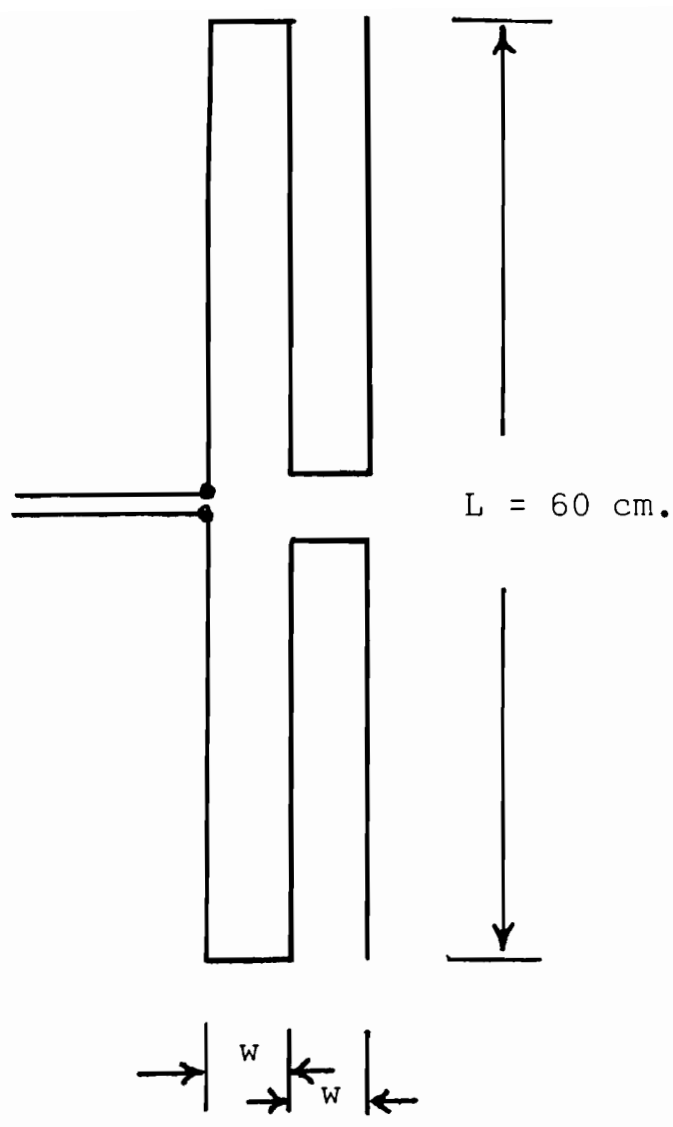

FIGURE 5.1: MEANDER DIPOLE GEOMETRY. $(\mathrm{N}=2)$ 
Four values of $w$ were used to model a meander dipole antenna. These are $w=0.5,1.0,2.5$ and $3.0 \mathrm{~cm}$.

The results are shown in table 5.1 , below.

TABLE 5.1: RESONANT RESISTANCE OF A MEANDER DIPOLE.

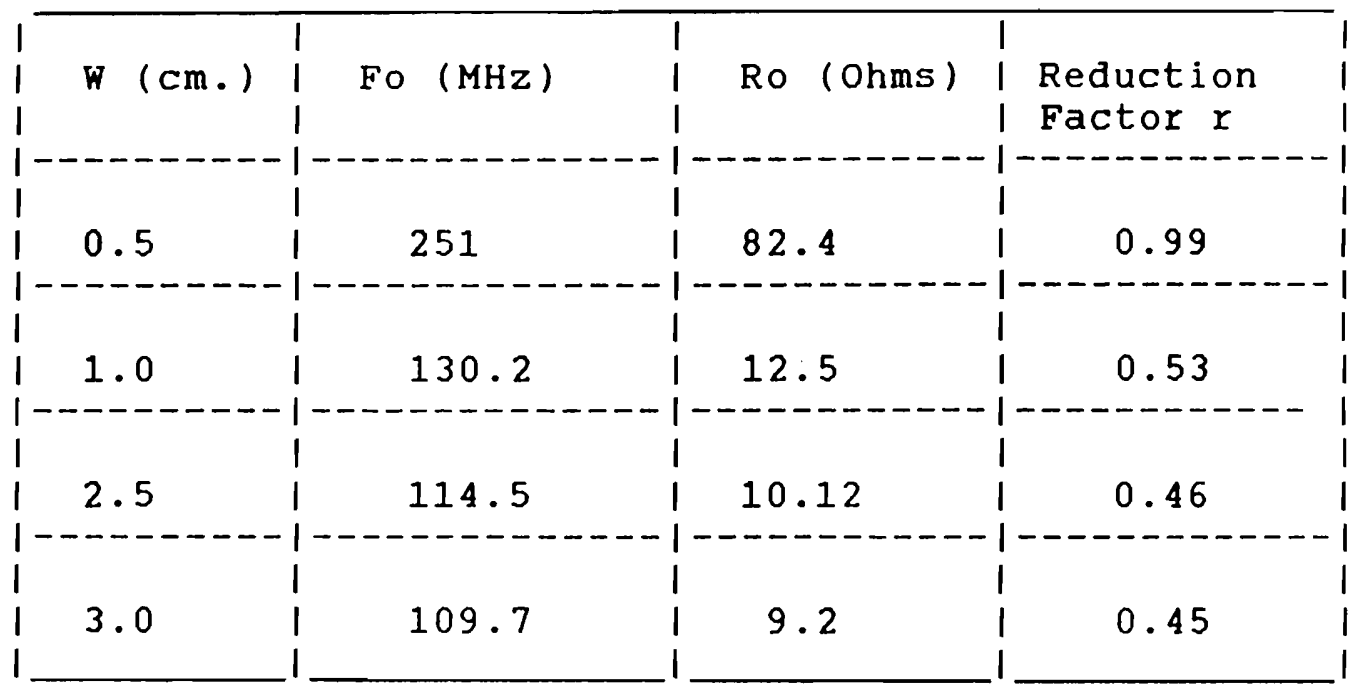

The above results reflect what we already know from previous analysis of meander monopoles:

- The resonant frequency tends to decrease as the wire separation $w$ is increased. This means an increase in size reduction ( reduction factor is smaller)

- The resonant resistance becomes smaller as $w$ increases.

However, MININEC yields some differences that are worth noting.

First, the reduction factor seems to be somewhat smaller than for monopoles over conducting ground. Let us remember that a typical value for the reduction factor $r$ was 0.6 for monopoles. MININEC results for dipoles show factors in the order of 0.4 to 0.5 .

In second place, the radiation resistance, although larger than for monopoles, remains at a relatively low value. From comparing meander dipoles with conventional dipoles/monopoles, it was expected that this resistance would be somewhat larger. In any case, the effect of $w$ in the value of this resistance is quite apparent. 
For the case, where $w=0.5 \mathrm{~cm}$, we see that the value of the resonant resistance is large, comparable to that of a conventional monopole. Also, the reduction factor is almost 1.0 (no sizereduction, . This no doubt signals an erronous result. This can be attributed to the fact that the wire separation value of $w=0.5$ might be too small for the program to yield accurate results.

on the other hand, the smaller the $w$, the more the antenna ressembles a conventional dipole ( the extreme case being $w=0$ ), and we can expect the results to tend to those for a conventional dipole, as w becames increasingly smaller.

The next step is to to determine the effect of changing w, on the antenna bandwidth. The same values of $w$ as shown in the previous table were analysed, and the corresponding geometries were subject to a frequency sweep around their resonant frequencies. Again, the bandwidth is determined on the basis of a VswR equal to 2.0 .

The results are shown in table 5.2. Also, flgures 5.2 and 5.3 show the smith-Chart representation of the results, for the case where $w=2.5 \mathrm{~cm}$. and $w=3.0 \mathrm{~cm}$.

TABLE 5.2: EFFECT OF.W ON MEANDER DIPOLE BANDWIDTH.

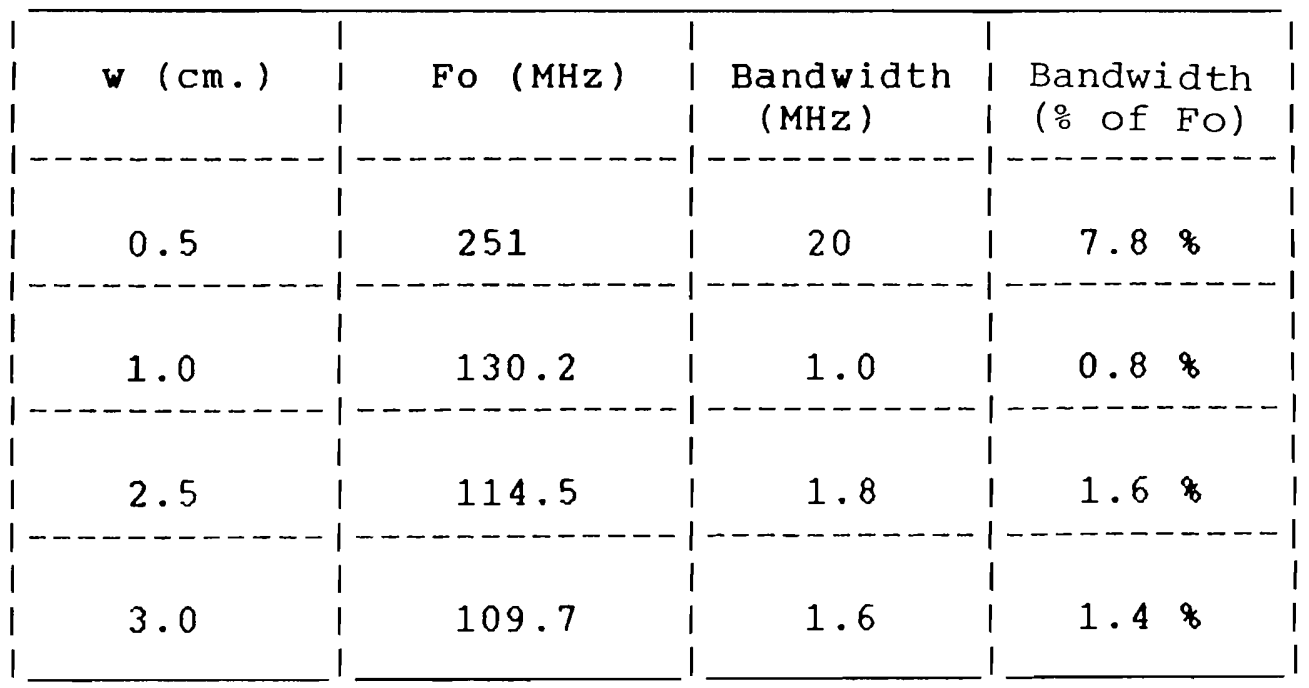

From these results, we can conclude that meander dipoles follow the expected behaviour already seen in meander monopoles. 
We can see that the bandwidth tends to decrease with increasing w. The value of bandwidth obtained for $w=1.0 \mathrm{might}$ be the result of a program error due the reasons stated previously ( w being too small compared to the wavelength to yield accurate results)

Although the value in $\mathrm{MHz}$. for the case $\mathrm{w}=2.5 \mathrm{~cm}$ is greater than that for $w=3.0 \mathrm{~cm}$., the bandwidth as a percentage of the resonance frequency is indeed less for the larger value of $w$.

Figures 5.2 and 5.3 give the smith chart representation of the meander dipole impedance for the case $w=2.5 \mathrm{~cm}$. and $w=3.0 \mathrm{~cm}$. The actual impedance values for the two cases are given in the following tables 5.3 and 5.4 .

TABLE 5.3: MEANDER DIPOLE $W=2.5 \mathrm{~cm}$. IMPEDANCES (MININEC)

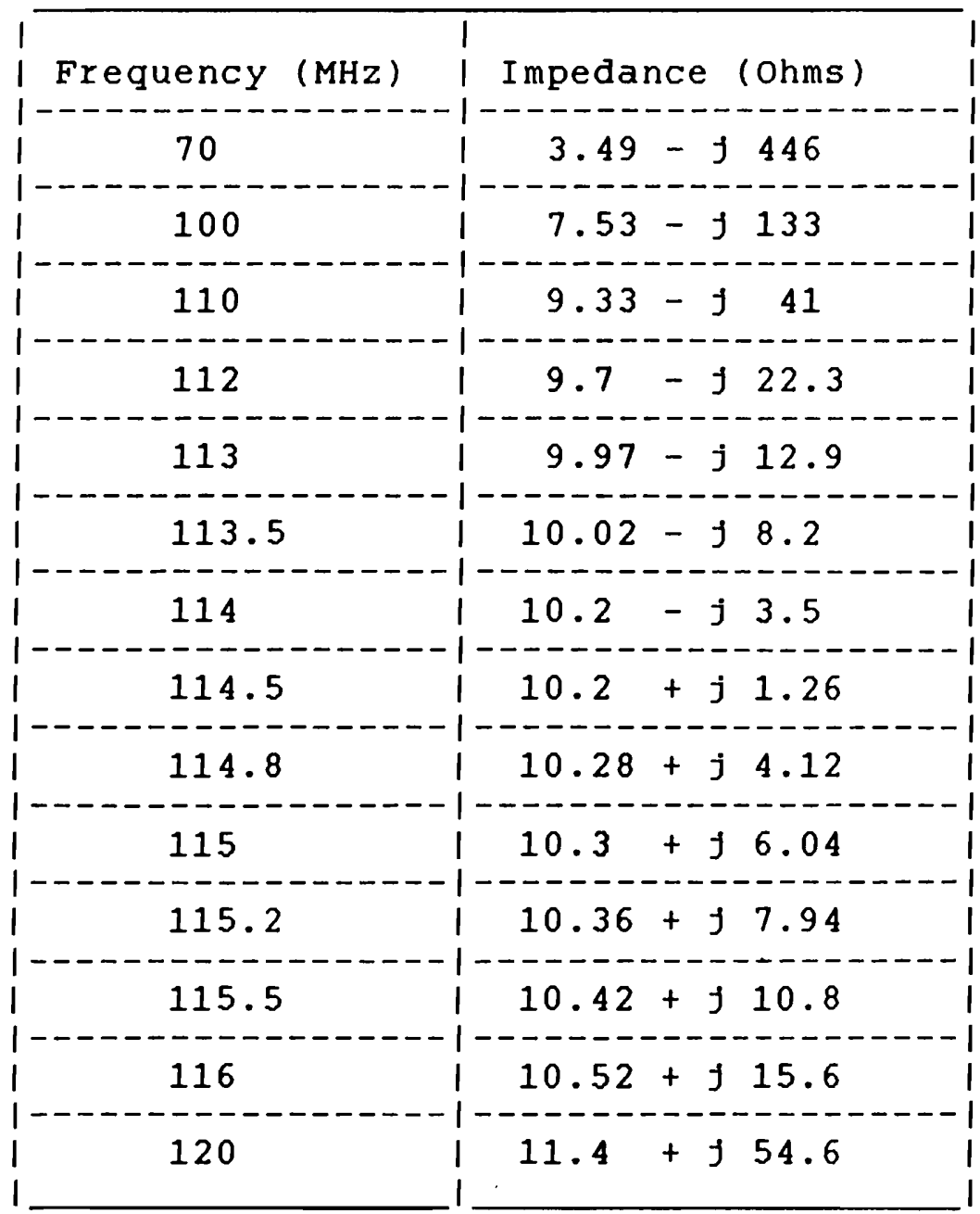




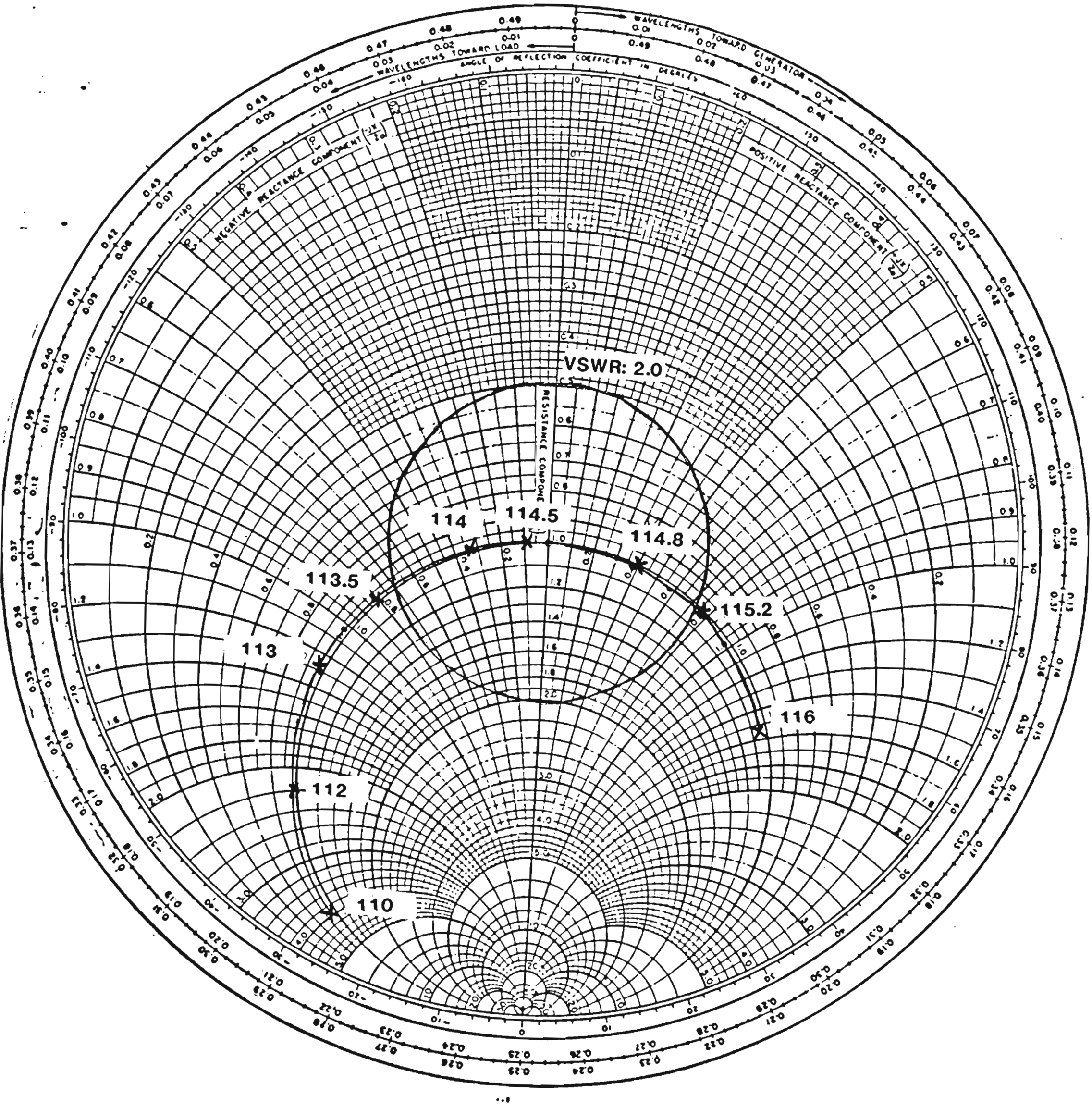

FIGURE 5.2 MEANDER DIPOLE ( $w: 2.5 \mathrm{~cm}$.) MPEDANCE CHART NORMALIZED TO RESONANT RESISTANCE 


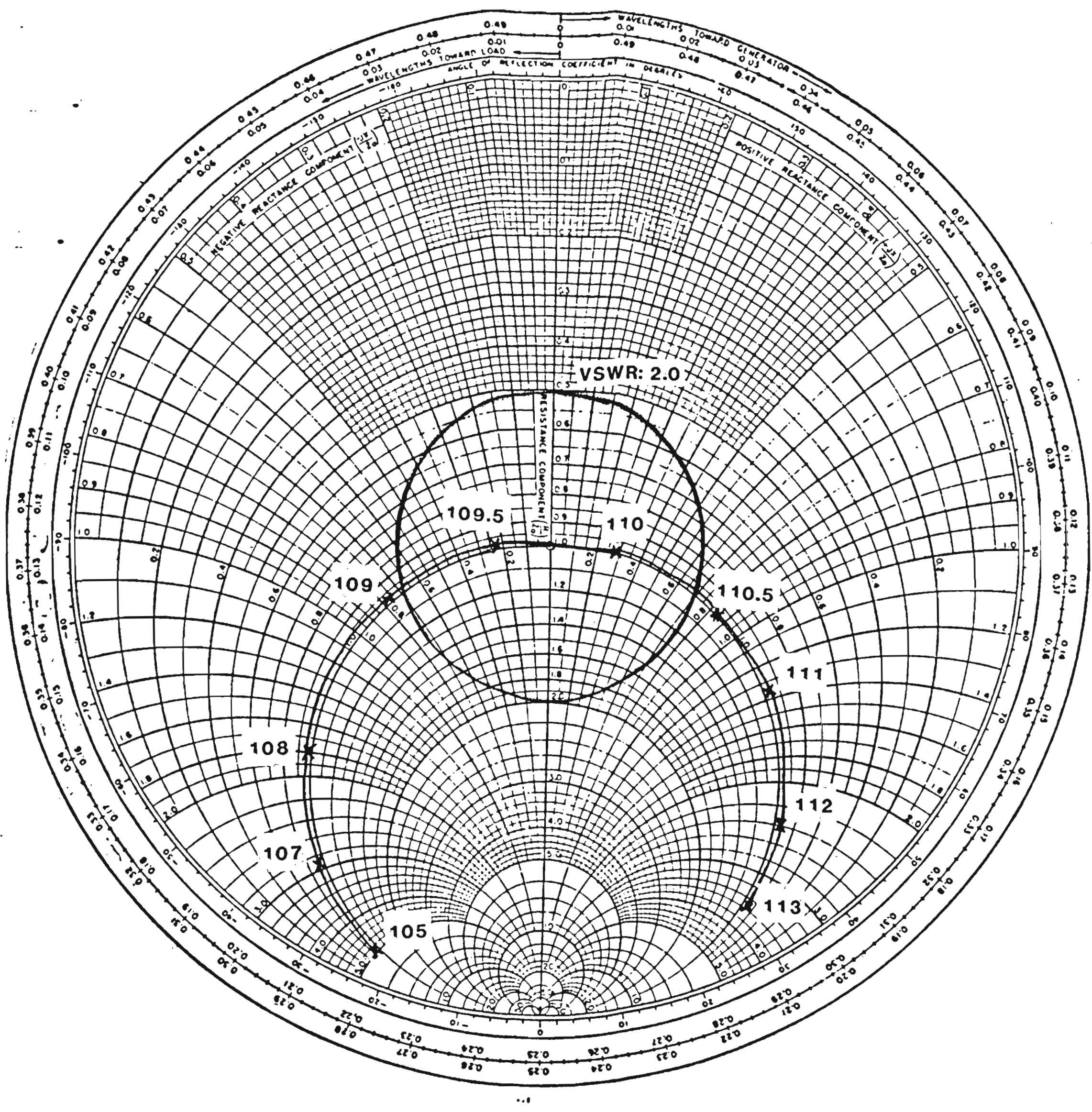

FIGURE 5.3 MEANDER DIPOLE ( $w: 3.0 \mathrm{~cm}$.) IMPEDANCE CHART NORMALIZED TO RESONANT RESISTANCE 
TABLE 5.4: MEANDER DIPOLE $W=3.0 \mathrm{~cm}$. IMPEDANCE (MININEC)

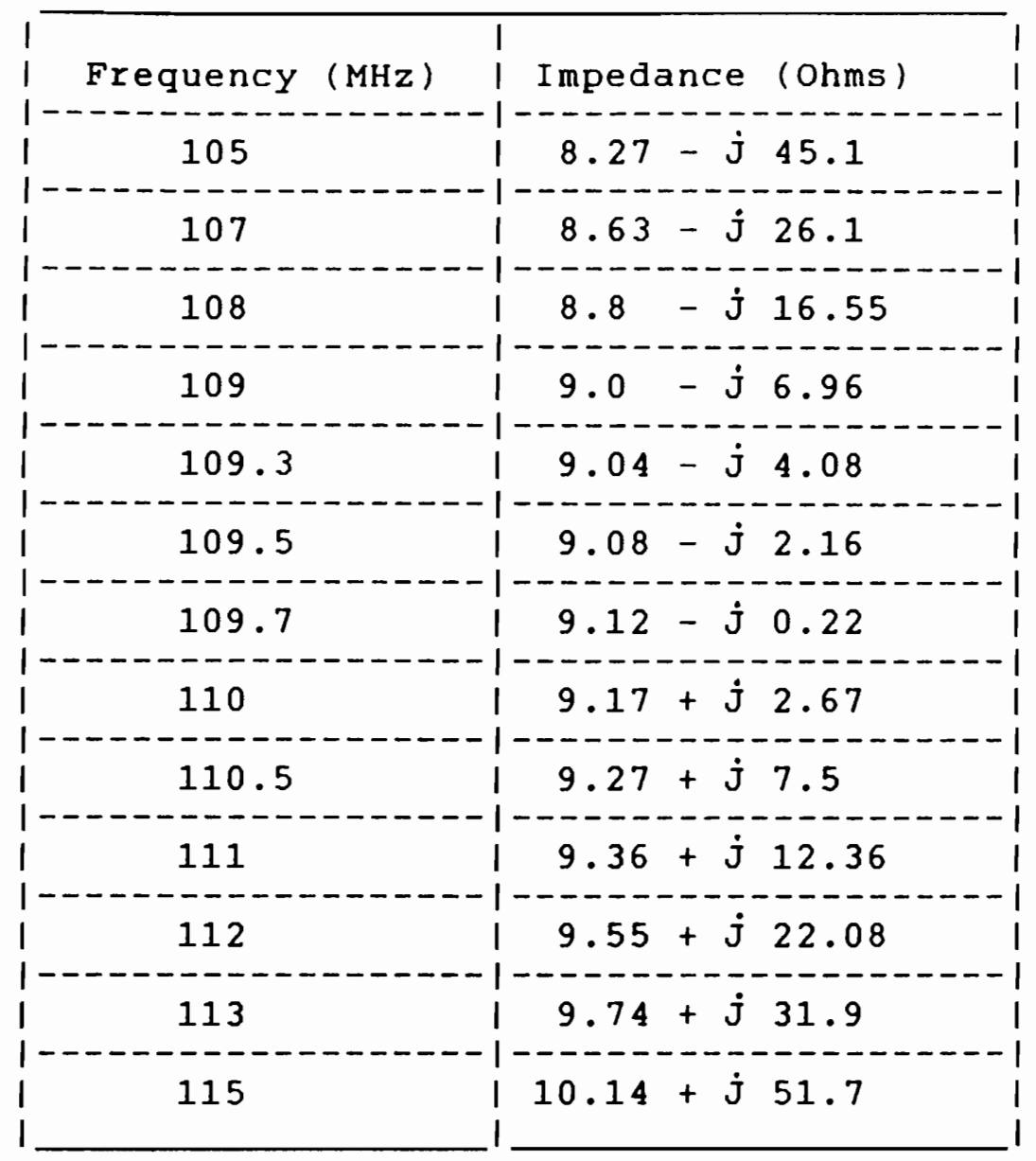

As we can see from these tables, and from the corresponding Smith Charts, the value of the resistance varies very little around resonance. The reactance, on the contrary, seems to dip very sharply at resonance. This, of course explains the narrow bandwidth that appears to be the predominant characteristic of meander antennas.

Although, as was pointed out, we do not have any experimental reference to compare these results with, it is obvious that they are in accordance with those obtained in chapters 3.0 and 4.0 , for meander monopoles.

These results serve as a good starting point, to begin the design of the folded meander dipole. In doing so, we are to assume that the properties of folded dipoles will somewhat be maintained by its meander counterpart, but with the basic characteristics of the meander dipole, studied in this section. 
A point that must be made, is that, in choosing the value of the wire separation $w$, it was intended to keep the overall "width" of the antenna small. It must be remembered (see fig. 5.1) that this antenna width is equal to twice the value of $w$.

The main feature of meander antennas is their reduced size. However, the "size" is not only the length L of the dipole. For a meander antenna, the wire separation w must also be considered.

For instance, if a considerable reduction in the length of a dipole was obtained, at the price of making w significantly large compared to the length, then we could not consider the overall size reduction to be very big. In a case like this, we would have to flnd another parameter, like the antenna aperture, to establish what the real reduction in overall size was.

In this context, the wire separation was kept small compared to the length, in order to have the overall "slze" reduction correspond as closely as possible to the reduction in length.

The general criteria adopted for this purpose, was that the total antenna width (equals two times $w$ ) was to be not larger than onetenth of the antenna length. That is:

$$
\text { or } \begin{aligned}
2 w & <0.1 L \\
w & <0.05 L
\end{aligned}
$$

This explains the cholce of the largest value of $w=3.0 \mathrm{~cm}$. for the meander dipole of length $\mathrm{L}=60.0 \mathrm{~cm}$.

Another aspect of meander dipoles that should be noted, is that the value of resonant resistance is somewhat smaller than what could be expected. In fact, the Impedances do not vary much from those presented in chapter 3.0 and 4.0 for meander monopoles.

If we compare these results to the case of conventional monopoles and dipoles, the differences are obvious. A conventional half-wave dipole resonant resistance is significantly greater than that of a monopole. This does not seem to be the case for meander dipoles.

Furthermore, the meander monopoles studied in previous chapters, had a length of only $4.5 \mathrm{~cm}$., whereas the values of wlre separation studied were baslcally the same: $w=0.5$ or $1.0 \mathrm{~cm}$. These values of $w$ represent a much greater fraction of the wavelength for the antenna $L=4.5 \mathrm{~cm}$., than for the dipoles studied here. Nevertheless, the values of resistance do not seem to differ much.

This phenomenon cannot be explained at the moment, since we do not posess experimental data on meander dipoles to serve as reference to our MININEC results. 
In the previous section, we have tried to model various meander dipoles with $\mathrm{N}=2$, and have found several results that could be inconsistent with everything we know experimentally about meander antennas. To begin with, let us sum these results:

- Resonant Resistance is comparable, and sometimes less than that of a meander monopole (refer to chap. 4) over conducting ground. This resistance was expected to be larger, if only because the wire is longer for the same resonant frequency. (meaning larger ohmic resistance.)

- Bandwidth is small, but is smaller than the ones found experimentally and with MININEC for meander monopoles.

- Behaviour of the meander dipole, as $w$ is changed, is the same as for meander monopoles. That is, bandwidth decreases with increasing $w$, and resonant resistance decreases.

- reduction factor is comparable, though somewhat smaller than for a meander monopole.

The geometry analysed in the previous section was composed of 46 segments, which is very near the limit MININEC will accept. Now, we have found (chap. 3.3 .2 ) that a meander dipole $N=2$ can be accurately modelled with a number of segments, that has to be appro$x i m a t e l y$ greater than 30 .

However, the meander dipole has a physical length of double the one of a meander monopole. A logical conclusion would be that the dipole requires at least 60 segments, for the program to yield accurate results. But we are limited by the maximum number of 50 segments imposed by the program, and thus we may be forced to accept a suboptimal solution.

Therefore, the results obtained with the present segmentation must be treated as valid approximation to the real solutions, but it is doubtful that experimental results will closely match the MININEC results.

We can affirm that the results are indeed valid, because as it has been pointed out, most of the characteristics found in the model of the meander dipole, agree with experimental data [2] for meander monopole antennas. It is only the accuracy of the numerical results such as: resonant resistance, bandwidth, and resonant frequency, that have to be taken as approximations. 
To further investigate this point, other segmentations were tried. First, the number of total segments was reduced to 40 . Then, the maximum number of segments $(50)$ allowed by MININEC was tried.

In this later case of a 50 segment dipole, segments were added to only the most critical wire, the longest wire where the feed-point is connected (ref. to fig. 5.1). The reason for this is that only 4 extra segments could be added to the orlginal 46-segment geometry, and segments could not be added to every wire in the geometry which has 9 wires in total. Adding 1 or 2 segments to some of the shorter wires would have created an "inbalance" in the antenna, with two symmetrical wires having different segmentation.

For these 3 cases, the resonant frequency and the resonant resistance were found. The results are as follows:

\begin{tabular}{ccc} 
No. of Segments & Res. Freg. (MHz) & Res. Reslstance (Ohms) \\
\cline { 2 - 3 } 40 & 220 & 59.8 \\
46 & 130.2 & 12.6 \\
50 & 168.5 & 19.6
\end{tabular}

Note that for the case of a 50 segment dipole, the resonant resistance has increased over the 46-segment resistance, and the resonant frequency has also increased to $168.5 \mathrm{MHz}$.

This represents a reduction factor of 0.67 , which is perhaps farther from the expected value of 0.5 to 0.6 , than the 0.53 reduction factor obtained previously for the 46 segment segment geometry. The impedance, by contrast, is maybe closer than the expected value (or higher than a for a monopole), which tells us that at least in this respect the result is closer to the real value.

The case of the 40 segment geometry gives us a higher value of resonant resistance, but the change in resonant frequency yields a reduction factor of 0.88 , which is in total disagreement with what we know about meander antennas.

It is worth noting that apparently, the only parameter that presents drastic changes with the segmentation, is the antenna input reactance Thus the change in resonant frequency. The input resitance seemed unchanged by the segmentation. For instance, the resistance at $130 \mathrm{Mhz}$. for the 40 and 50 segment cases, remalned roughly at the 46-segment value of 12.6 ohms.

The point that must be made, is that probably all of this segmentations are inadequate to predict exact real-life results. Which is why, again, sald results are valid only as qualitative, rather than quantitative approximations, due to MININEC limitations. 
In this section, we will try to study the characteristics of the meander folded dipole through a valid MININEC model.

The basic geometry we are modelling is described in figure 5.4. We can see that this is a rather complex geometry. It is formed by two meander dipoles $\mathbf{N}=2$, connected and separated by a distance equal to d. Or it could also be seen as a meander dipole $N=4$ folded over itself.

The antenna is composed of 20 separate wires. The segmentation density, as well as the geometry and the coordinates in space for each wire will be given in detail here.

The antenna was chosen to have a vertical orientation. That is, the length $L$ of the dipole will be along the vertical axis of coordinates (The $\mathrm{z}$ axis.)

The segmentation density used is 50 segments. We must remember that the limit number of segments that MININEC will accept is 50 .

However, when we distribute this number of segments over 20 different wires, the segment density per wire tends to be small. This could entall erronous or marginal results, but given the constraints of the program and the complexity of the antenna, we have no other option.

We can say in this respect, that we are forcing the program to its very $11 \mathrm{mits}$.

Again, in this case, as we are dealing with a new type of antenna, there is no previous experimental data to give us a reference on the accuracy of the results. Nevertheless, we have gathered sufficient data on meander antennas and folded dipoles to be able to determine if the results are correct, or the program is yielding numerically erroneous results.

Again, in trying to keep the dimensions of the antenna, similar to those of a conventional folded dipole, the values of $w$ and the arm separation d, were kept sufficiently small so that the overall width of the antenna was a small fraction of the length $\mathrm{L}$.

We must remember that in the case of a folded meander dipole, the overall antenna width will be:

$$
\text { width }=4 w+d \text {. }
$$

In this case, the criteria used was that the width should not be greater than $25 \%$ of the antenna length, or

$$
4 w+d<0.25 L
$$




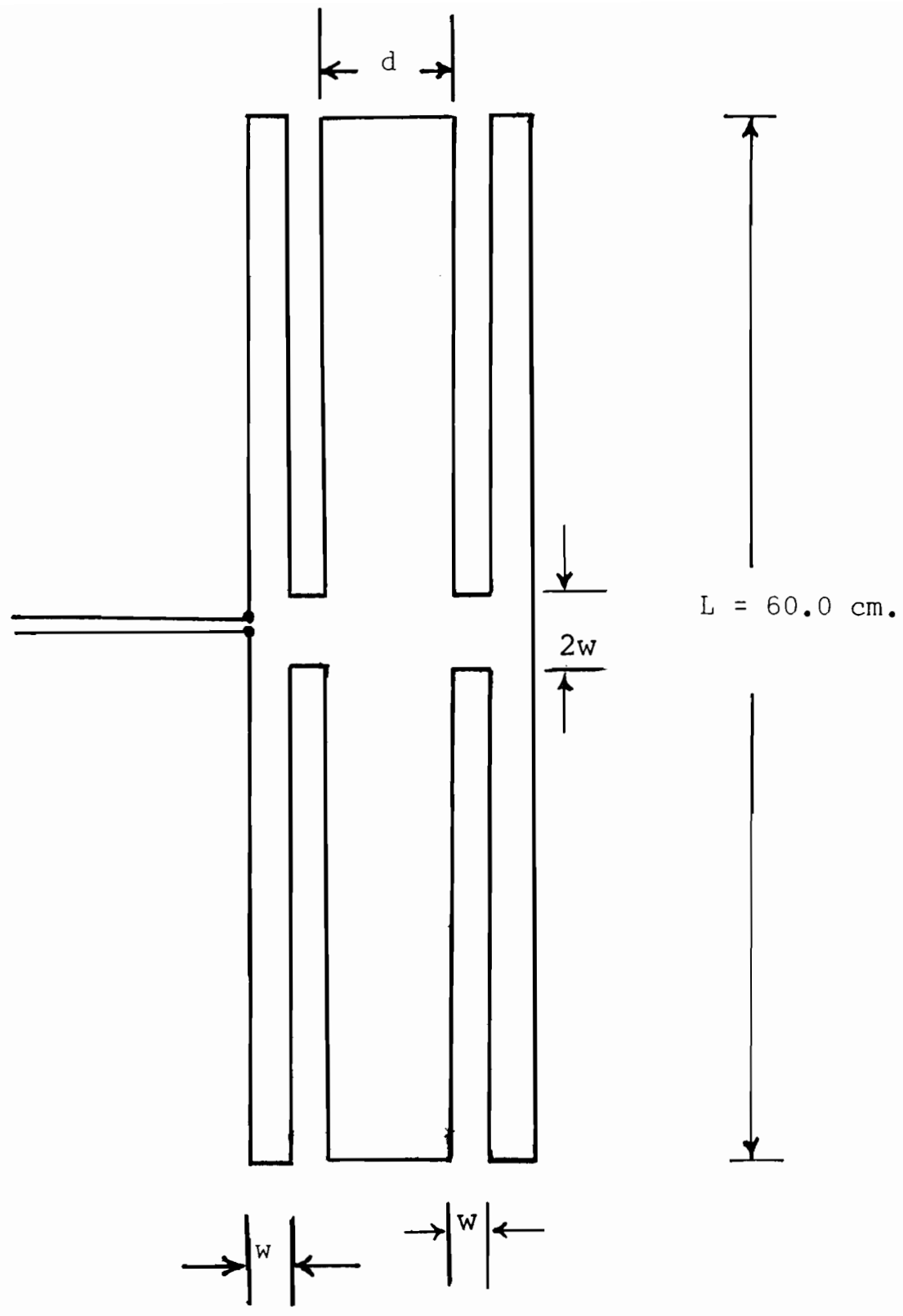

FIGURE 5.4: MEANDER FOLDED DIPOLE GEOMETRY. $(\mathrm{N}=2)$ 
The length of the arms was inltially set at $L=60 \mathrm{~cm}$., to match the length chosen for the meander dipoles of the previous section.

The following table gives the values of the wire coordinates, as well as the segmentation for each wire, for a meander folded dipole of length $\mathrm{L}=60 \mathrm{~cm} ., \mathrm{w}=1.0 \mathrm{~cm}$, and $\mathrm{d}=4.0 \mathrm{~cm}$.

TABLE 5.5: FOLDED MEANDER DIPOLE COORDINATES $(\mathrm{cm}$.

\begin{tabular}{|c|c|c|c|c|c|c|c|}
\hline WIRE No. & $\begin{array}{r}\text { END } \\
\times 1 \\
\end{array}$ & $\begin{array}{c}\text { COORL } \\
\mathrm{Y} 1 \\
\end{array}$ & $\begin{array}{c}\text { ATES } \\
\text { Z1 }\end{array}$ & $\begin{array}{r}\text { END } \\
\times 2 \\
\end{array}$ & $\begin{array}{c}\text { COORD } \\
\mathrm{Y} 2\end{array}$ & $\begin{array}{l}\text { ATES } \\
\text { Z2 }\end{array}$ & SEGMENTS \\
\hline 1 & 0 & 0 & 0 & 0 & 0 & 60 & 8 \\
\hline 2 & 0 & 0 & 60 & 1 & 0 & 60 & 1 \\
\hline 3 & 1 & 0 & 60 & 1 & 0 & 31 & 3 \\
\hline 4 & 1 & 0 & 31 & 2 & 0 & 31 & 1 \\
\hline 5 & 2 & 0 & 31 & 2 & 0 & 60 & 3 \\
\hline 6 & 2 & 0 & 60 & 6 & 0 & 60 & 1 \\
\hline 7 & 6 & 0 & 60 & 6 & 0 & 31 & 3 \\
\hline 8 & 6 & 0 & 31 & 7 & 0 & 31 & 1 \\
\hline 9 & 7 & 0 & 31 & 7 & 0 & 60 & 3 \\
\hline 10 & 7 & 0 & 60 & 8 & 0 & 60 & 1 \\
\hline 11 & 8 & 0 & 60 & 8 & 0 & 0 & 8 \\
\hline 12 & 8 & 0 & 0 & 7 & 0 & 0 & 1 \\
\hline 13 & 7 & 0 & 0 & 7 & 0 & 29 & 3 \\
\hline 14 & 7 & 0 & 29 & 6 & 0 & 29 & 1 \\
\hline 15 & 6 & 0 & 29 & 6 & 0 & 0 & 3 \\
\hline 16 & 6 & 0 & 0 & 2 & 0 & 0 & 1 \\
\hline 17 & 2 & 0 & 0 & 2 & 0 & 29 & 3 \\
\hline 18 & 2 & 0 & 29 & 1 & 0 & 29 & 1 \\
\hline 19 & 1 & 0 & 29 & 1 & 0 & 0 & 3 \\
\hline 20 & 1 & 0 & 0 & 0 & 0 & 0 & 1 \\
\hline
\end{tabular}


This geometry is far from the ideal one. The most critical wires in this antenna are those containing only 3 segments. For a wavelength of 2 meters $(150 \mathrm{MHz})$, one of these segments will only be 0.048 or $5 \%$ of the wavelength. Although theoretically, this is within the limits of the programs, the results may be inaccurate. In any case, there was no way of knowing if such results were erroneous, until they could be verified with practical laboratory results.

However, we could make some preliminary assumptions about this antenna, that could serve as a basis for determining the validity of the results.:

1- The antenna was expected to have somewhat greater value of impedance at resonance than the meander dipole.

2- The bandwidth, of course, was expected to be at least as narrow as a meander dipole. Furthermore, considering that a folded dipole has a smaller bandwidth that a conventional dipole, it was thought very possible to obtain an even smaller bandwidth for this antenna, than for a meander dipole.

3- The overall impedance change with frequency, should correspond to that of a loop antenna or a folded dipole. This means, the antenna will have a first resonance point characterized by a very high resonant resistance and sharp reactance change.

4- A change in wire diameter in the second "arm" of the antenna, with reference to the feeding-point arm, should somewhat correspond to a change in input impedance.

This antenna was subject to basically the same tests with MININEC as those previously accomplished for meander monopoles and dipoles.

A voltage source of 1.0 Volts was applied to the center of wire No. 1, onto segment No. 3 .

First, the antenna was subject to a frequency sweep to determine both its second resonance resitance and also to verify the loop antenna characteristics mentioned in our basic assumption No. 3 above.

The results given by MININEC for this first geometry are quite encouraging. In the first place, the input resistance is close to 50 ohms. (one of our target impedances.) at second resonance. Furthermore, the value is roughly 4 times that of a meander dipole with the same values of $w$ and $L$.

Second, the impedance behaves much like that of a conventional folded dipole. The results are shown in the next page and are also shown in graphical form in figures 5.5 (Resistance) and 5.6 (Reactance). Note the similarity with fig. 4.22 and 4.23 


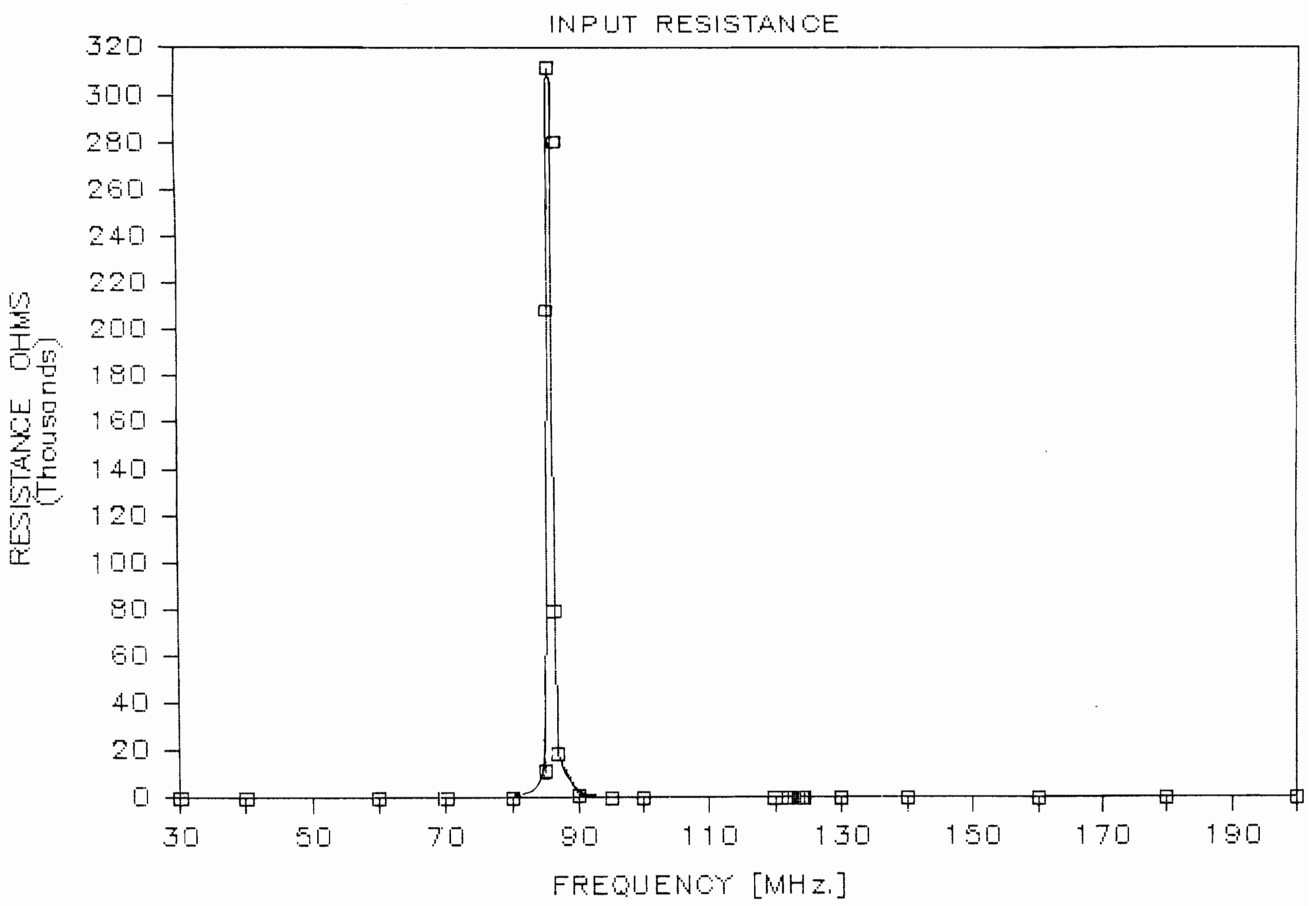

FIGURE 5.5: FOLDED MEANDER DIPOLE INPUT RESISTANCE $(w=1.0 \mathrm{~cm}$. VERSUS FREQUENCY. 


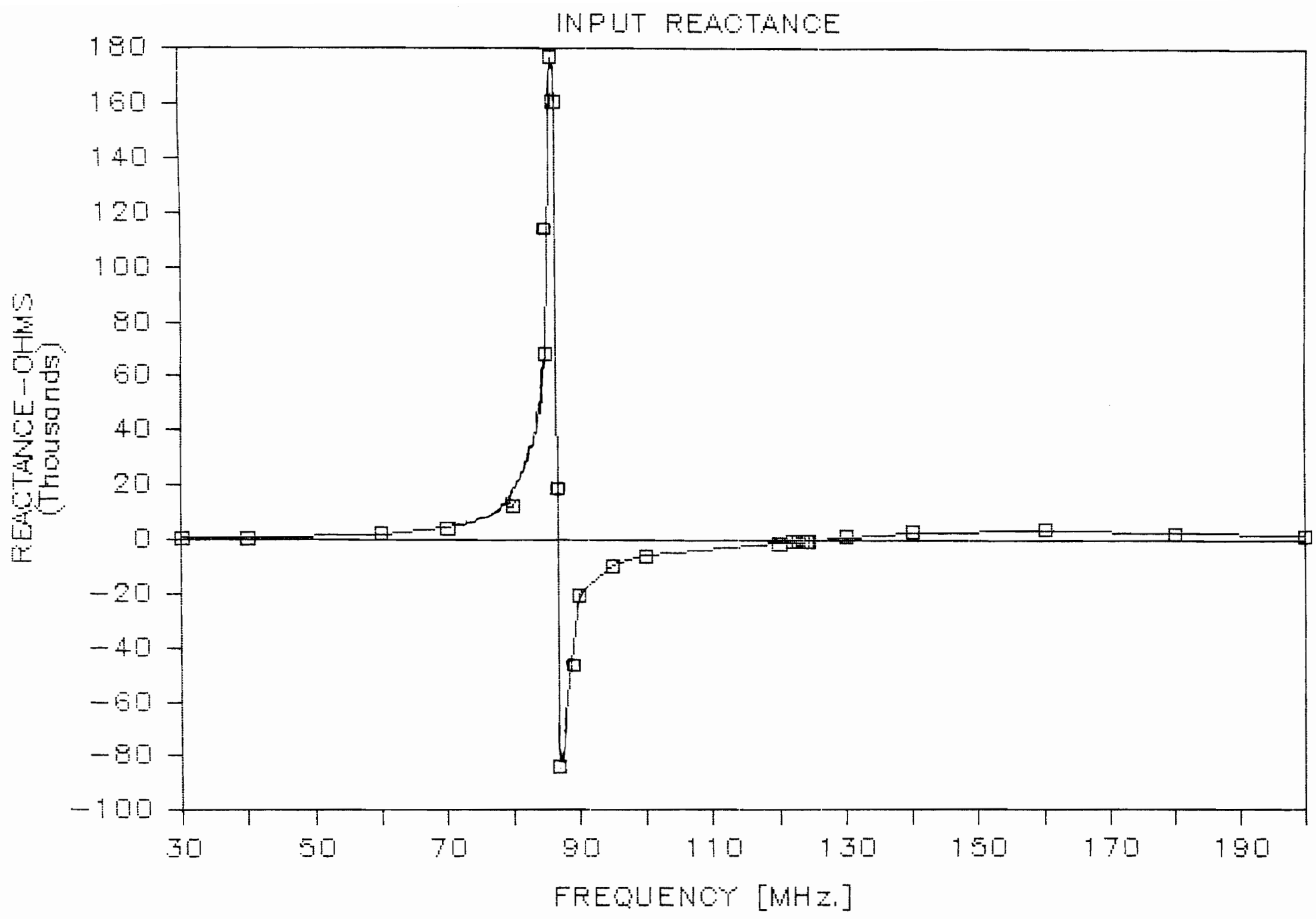

FIGURE 5.6: FOLDED MEANDER DIPOLE INPUT REACTANCE $(w=1.0 \mathrm{~cm}$. VERSUS FREQUENCY. 
TABLE 5.6: MEANDER FOLDED DIPOLE GEOMETRY INPUT IMPEDANCE 50 segment Geometry . $w=1.0 \mathrm{~cm} ., L=60 \mathrm{~cm}$.

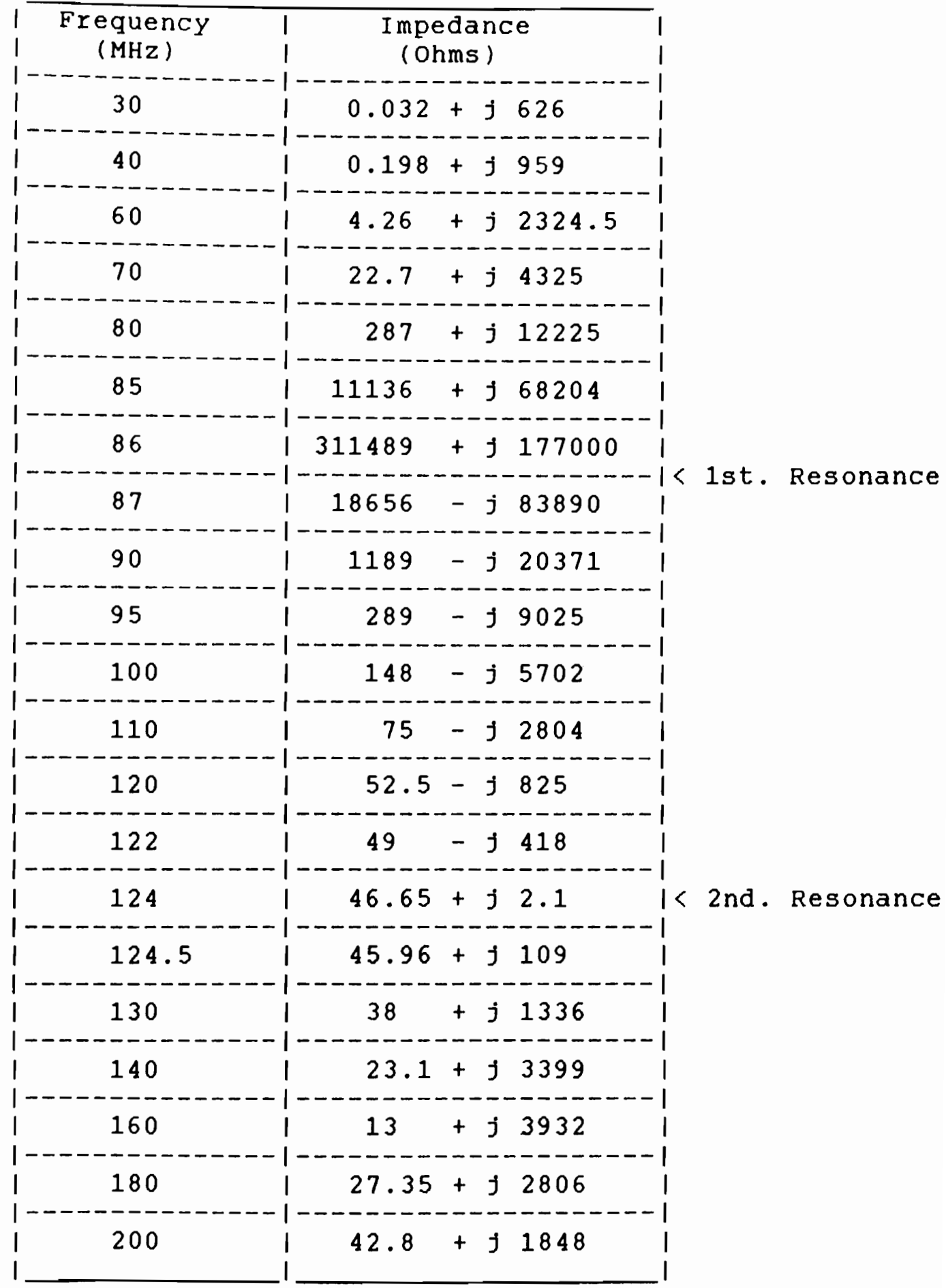


The next step was to analyse the bandwidth of this antenra. A more fine frequency sweep around the point of second resonance was accomplished.

The results have been plotted in the $5 \mathrm{mith}$ chart of figure 5.7 and in the Table 5.7.

Agaln, the bandwidth is calculated using the resonant resistance as normalization factor, and a VSWR circle of value 2.0

TABLE 5.7: FOLDED MEANDER DIPOLE IMPEDANCE AROUND 2nd. RESONANCE

\begin{tabular}{cc} 
FREQUENCY & IMHz) \\
\hline 123.0 & $48.0-\mathrm{j} 209.7$ \\
123.5 & $47.4-\mathrm{j} 104$ \\
123.7 & $47.1-\mathrm{j} 61.74$ \\
123.8 & $46.9-\mathrm{j} 40.5$ \\
123.9 & $46.8-\mathrm{j} 19.15$ \\
124.0 & $46.65+\mathrm{j} 2.14$ \\
124.1 & $46.52+\mathrm{j} 23.5$ \\
124.2 & $46.4+\mathrm{j} 44.93$ \\
124.5 & $45.96+\mathrm{j} 109.1$
\end{tabular}

It was found that the bandwidth is about $0.25 \mathrm{MHz}$. Th1s is in accordance with the expected results. A meander dipole with the same dimensions for $w$ and $L$, had a bandwidth of roughly $1.0 \mathrm{MHz}$. The folded meander dipole seems to present an additional reduction in bandwidth, Just as a folded dipole has a smaller badwidth than the conventional dipole.

This bandwidth seems too small. However we must remember that it was defined on the basis of a VSWR equal to 2.0 , which is a very stringent requirement. There are other methods of defining the bandwidth which will be discussed later.

Also note that the reduction factor (over a conventional folded dipole) is close to 50\%. This represents a small gain in reduction over the theoretical reduction obtalned with MININEC for a meander dipole in section 5.1 .

But we also know that a folded dipole is resonant at a slightly shorter length than a dipole, so this increase in reduction was to be expected. 


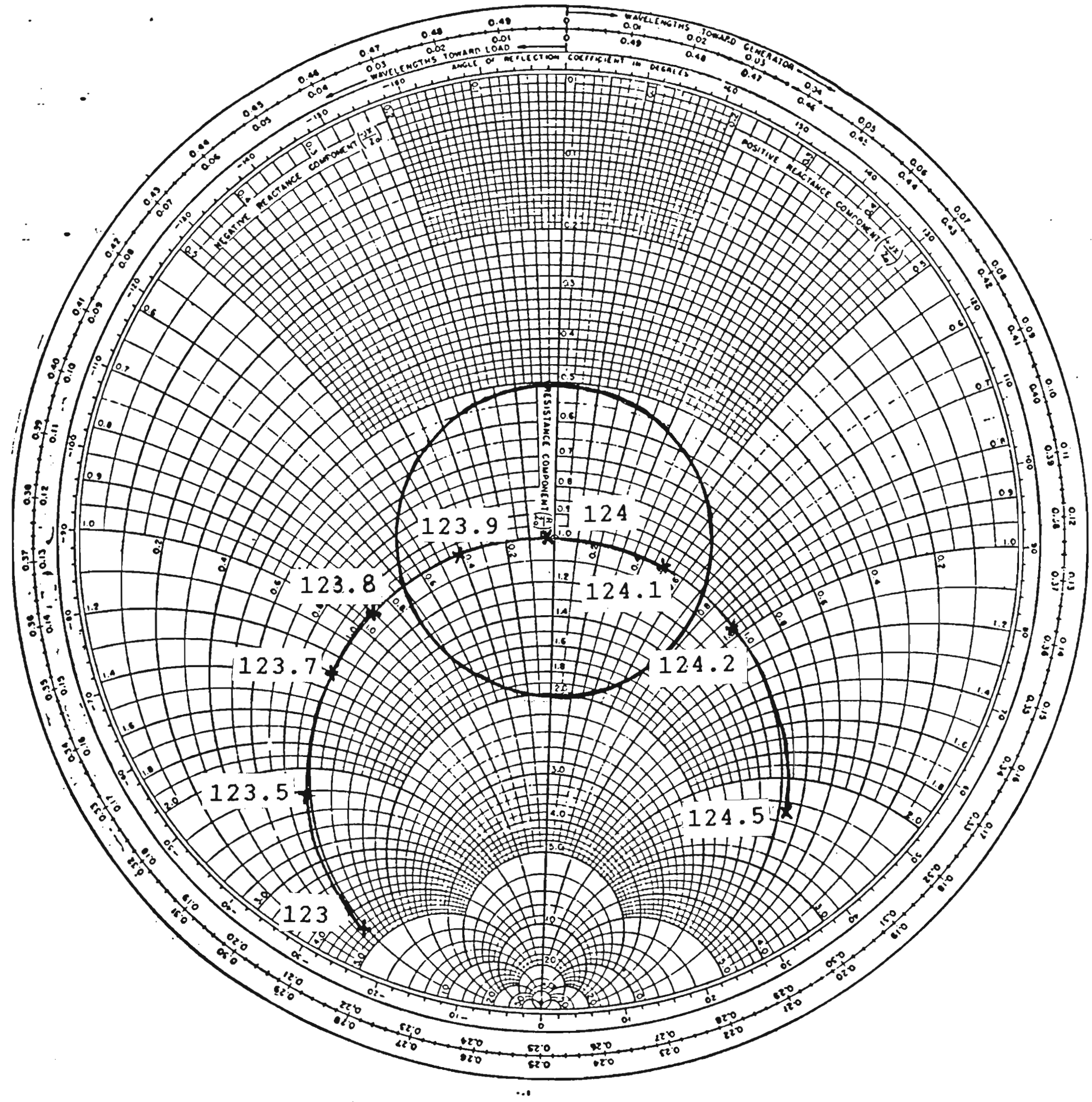

FIGURE 5.7 MEANDER FOLDED DIPOLE $(w: 1.0 \mathrm{~cm}$ ) MMPEDANCE CHART

NORMALIZED TO RESONANT RESISTANCE 
5.4.1 EFFECT OF WIRE SEPARATION IN ANTENNA CHARACTERISTICS:

The next test, was to increase the wire separation (w) of the meander arms. The arm separation (d) was kept constant at $4.0 \mathrm{~cm}$. to isolate the effect of varying $w$.

In choosing the maximum value of $w$, the criteria for maintaining the overall antenna width within specified limits was considered. Therefore, a maximum value of $w=2.0 \mathrm{~cm}$ was taken.

The geometry, and its corresponding wire segmentation was otherwise kept unchanged, as well as the excitation source and feed-point.

The results are shown in Table 5.8

TABLE 5.8: EFFECT OF $W$ IN ANTENNA RESONANT RESISTANCE d $=4.0 \mathrm{~cm} ., L=60 \mathrm{~cm}$.

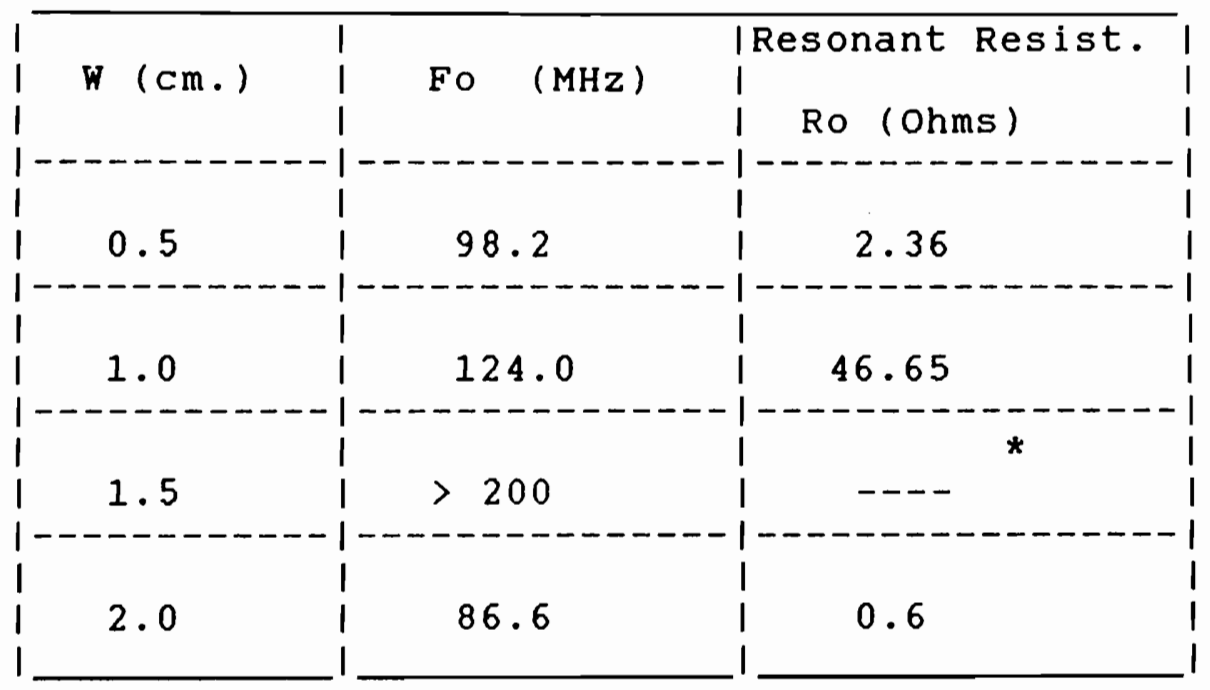

* : Freq. of second resonance is greater than $200 \mathrm{MHz}$.

These results do not show any obvious trend. The resonant frequency increases from the value corresponding to $w=0.5 \mathrm{~cm}$. to $124 \mathrm{MHz}$ for $w=1.0 \mathrm{~cm}$. , to a value beyond $200 \mathrm{MHz}$, and then comes back to $86.6 \mathrm{MHz}$. for $w=2.0 \mathrm{~cm}$. If anything, the resonant resistance seems to do something similar, starting at 2.36 ohms, increasing to 46 ohms, and then decreasing to 0.6 ohms. 
Similarly, the effect of wire separation in antenna bandwidth was analysed. From all previous analysis of meander antenna geometries, we know that bandwidth should decrease with increasing w.

The regults of the MININEC Analysis are shown in table 5.9.

TABLE 5.9: MEANDER FOLDED DIPOLE BANDWIDTH

$(\mathrm{L}=60 \mathrm{~cm}, \mathrm{~d}=4.0 \mathrm{~cm}$.)

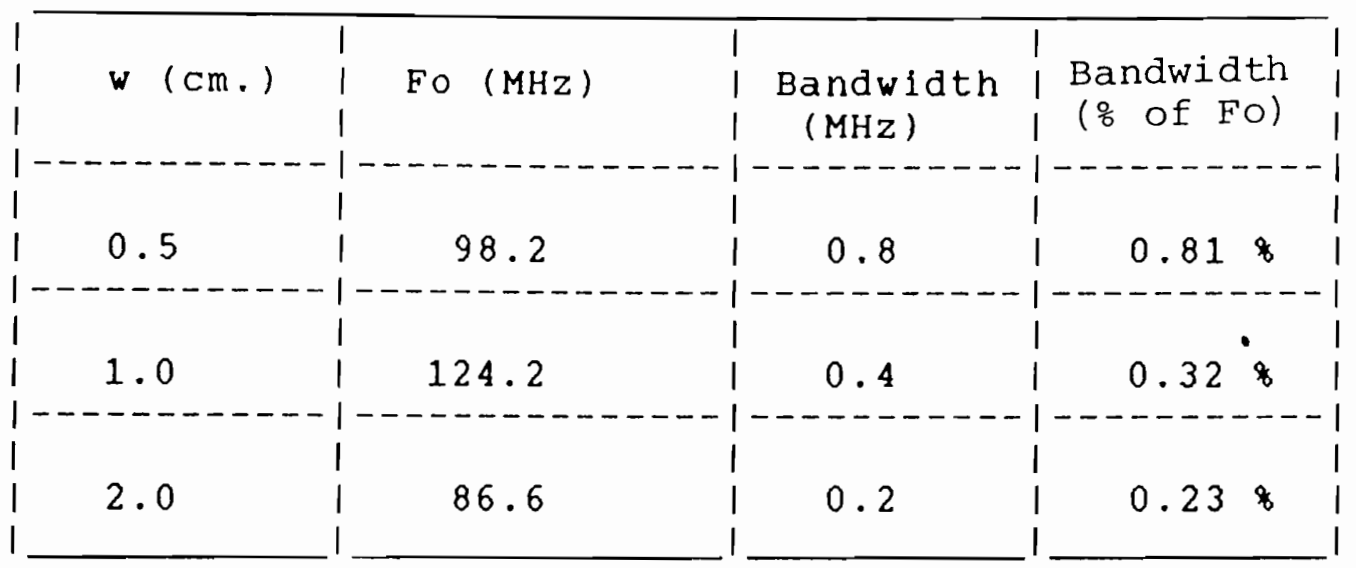

MININEC show two important features:

- The bandwidth shows the expected trend of being smaller with increasing $w$. Also, the bandwidth is smaller than that of a meander dipole. This is an expected result, since a folded dipole will have a smaller bandwidth than a conventional dipole.

- The resonant erequency, as was pointed out before, does not show the expected results of higher frequency with increasing w (higher reduction factor)

These discrepancies, specially where the resonant frequency, and therefore the resonant resistance are concerned, are again the product of a poor segmentation scheme, that is forced upon the designer by the program's inherent limitations.

We must stress the fact that all these results are to be taken at this point as qualitative approximations of what the antenna realIife behaviour will be. As was shown in section 5.2, the segmentation density is inadequate to yield exact numerical results. 
In previous chapters, radiations patterns of known antennas have been shown, as computed by MININEC. The program accomplishes this by calculating the electromagnetic fleld components around the antenna, in the far-field region. The theroretical details of this method will not be discussed here, but can be found in reference (1).

The program will let the user select wether the computation is to be done in volts/meter or dBi. If volts/meter is selected, the power level can be changed, and the radial distance of the far field calculation must be specified in meters. The zenith or vertical angle (theta) and azlmuthal or horizontal angle (phi) are then selected for both the volts/meter and dBi cases.

The results for the dBi calculation are presented in terms of the vertical pattern, horizontal pattern, and total pattern in $d B$. The total pattern is the vector sum of the two pattern components in $d B$.

In computing the far field components, the input impedance of the antenna is used to calculate the gain. Therefore, a well converged solution, and hence an accurate impedance result must be used, to obtain accurate gain data.

Now, we have shown that our solutions for the folded meander dipole antenna are most likely not very accurate, due to the poor segmentation density. Therefore, we must assume that the gain, as computed by MININEC will undoubtly introduce some degree of error.

However, we are interested in the qualitative results. That is, it is important to find out if this geometry will yield a pattern that is somewhat practical. Most important, it would be advantageous to find if this antenna has a pattern ressembling that of a conventional folded dipole.

The folded meander dipole having a $w=1.0 \mathrm{~cm}, L=60 \mathrm{~cm}$. and $d=4.0 \mathrm{~cm}$. was used for the pattern calculations by MININEC. The 50 segment geometry described previously was used.

MININEC yielded a pattern that is in every way shaped like that of a conventional folded dipole. Figure 5.8 shows the vertical radiation pattern. The pattern was calculated at an azimuthal angle of 45 degrees. The zenith angle theta was varled at 10 degrees intervals for a total of 36 angles ( 360 degrees.). The cholse of this number of vertical angles is obvious, since the antenna is suspended in free space.

The pattern, as shown if fig. 5.8 shows the familiar doughnut-shaped radiation pattern, that is the characteristic of dipoles and folded dipoles. Note that the maximum gain, as shown in the listing of results, is roughly $0.6 \mathrm{dBi}$, for the vertical pattern. However this number cannot be considered valid, since we are assuming that the input impedance is only an approximation. 
FIGURE 5.8 FOLDED MEANDER DIPOLE VERTICAL RADIATION PATTERN

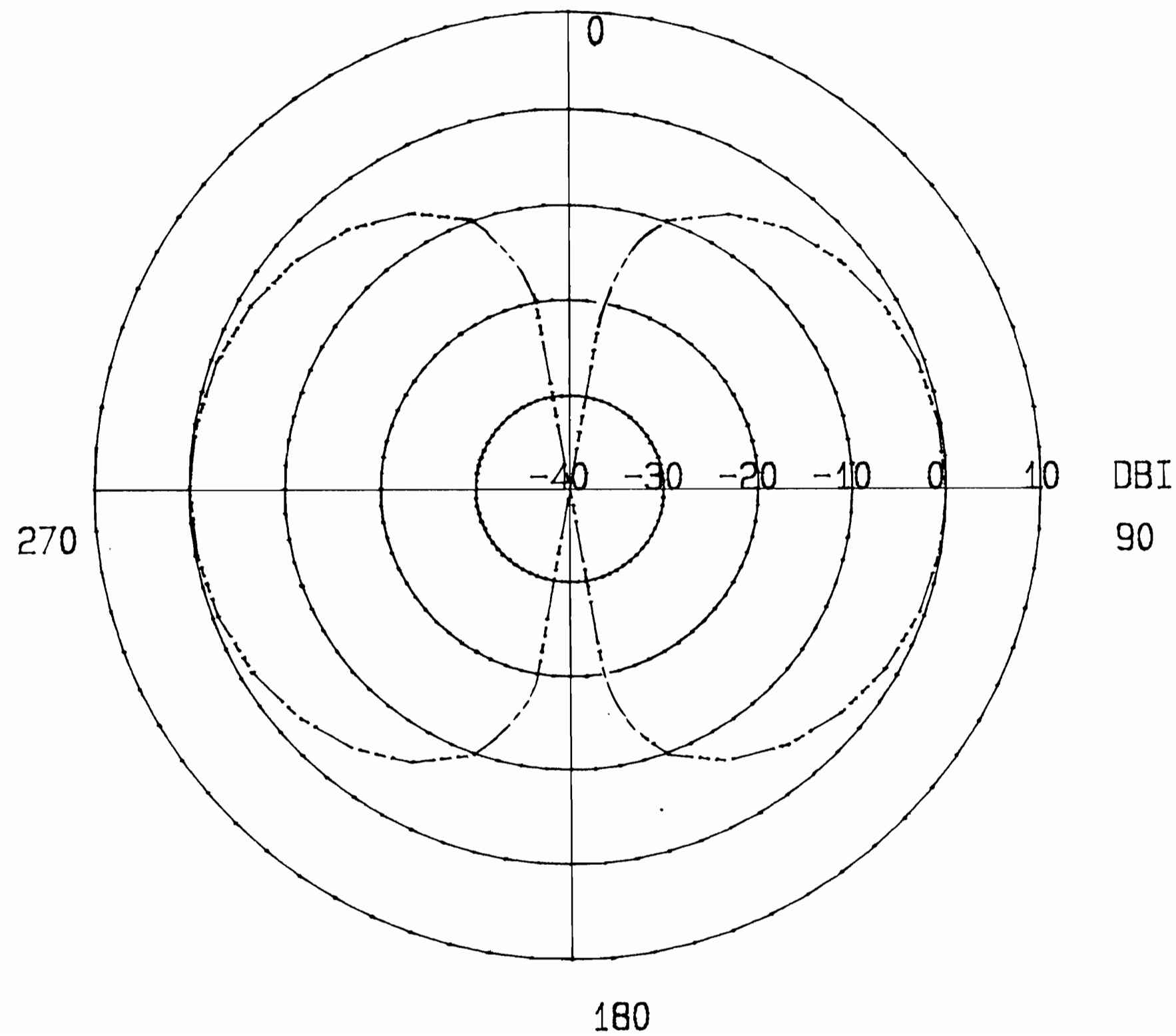


One of the most important characteristics of folded dipoles is the impedance transformation ratio. Since one of the reasons for choosing a folded dipole geometry for our meander antenna, was the impedance adaptability, it was necessary to test this feature with MININEC.

To accomplish this test, the wire-radius of one arm of our folded dipole was kept constant at $1.0 \mathrm{~mm}$., while the second arm, the one opposing the feed-point, was changed.

The results, which are shown in Table 5.9, confirm the fact that in a folded meander dipole, the impedance can indeed be adjusted by varying the diameter of the opposing meander arms.

The geometry chosen was the one previously described, w1th L $=60 \mathrm{~cm}$. $w=1.0 \mathrm{~cm}$. and $d=4.0 \mathrm{~cm}$, and a wire radius of $1.0 \mathrm{~mm}$. This geometry yields an input resonant resistance of 46.6 ohms, for identical wire radius o both arms.

TABLE 5.9: IMPEDANCE TRANSFORMATION RATIO AS A FUNCTION OF CONDUCTOR DIAMETER RATIO.

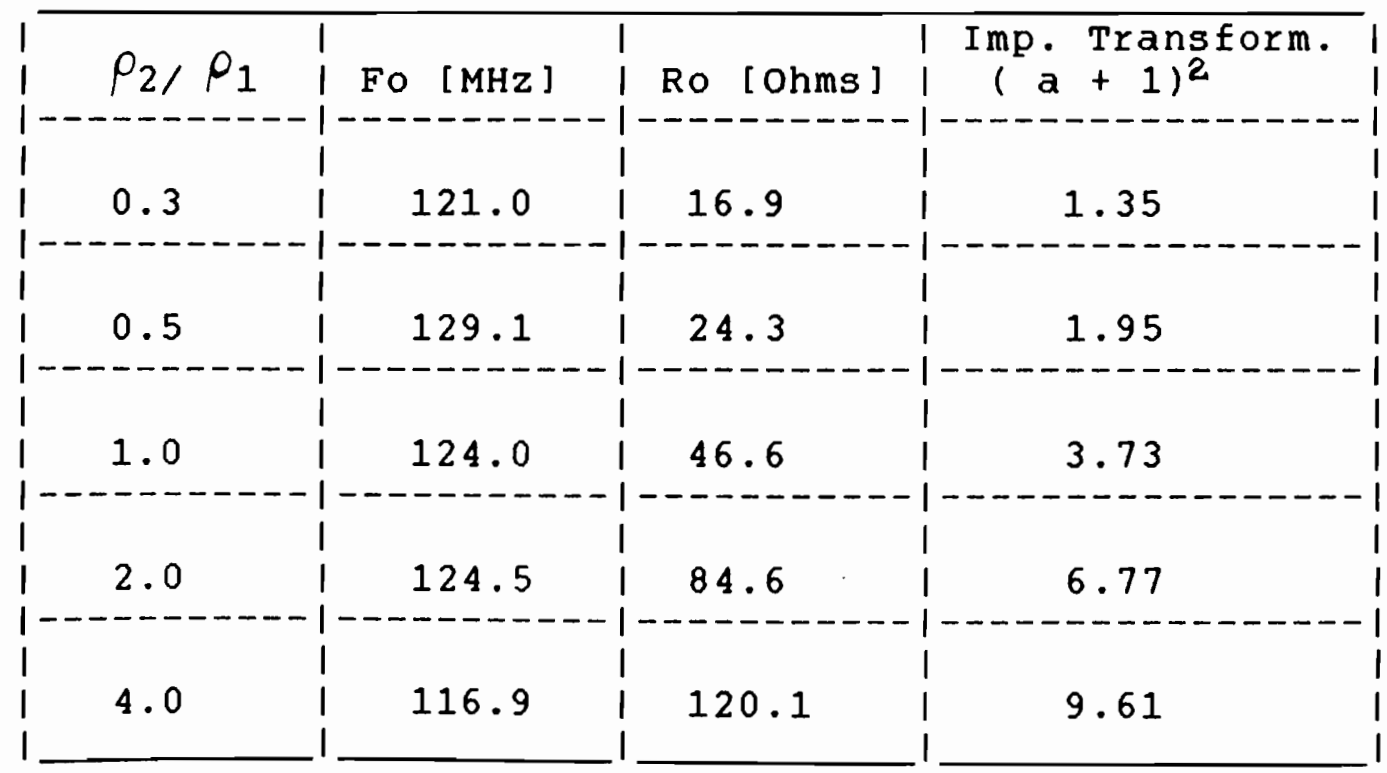


Table 5.9 shows the variation in antenna resonant resistance as the diameter of the second arm is varled. The impedance transfortion ratio was calculated taking as reference the the impedance of the meander dipole described in section 5.2, having a length of $60 \mathrm{~cm} ., w=1.0 \mathrm{~cm}$., for a resonant resistance of $12.5 \mathrm{ohms}$.

We can see that results are constrained to only a narrow range of radius ratios.

The reason for this is that the program will yield erroneous results ( such as negative input resistance) for ratios greater than 4.0 or smaller than 0.25 . This limits our abllity to draw general conclusions about the values of impedance transformation ratio on a folded meander dipole. However, the test proves very well the point that the impedance is, indeed, adjustable in this type of antenna by means of the change in conductor diameter.

It is quite apparent that the impedance of the meander folded dipole does follows the same trend of a conventional folded dipole, when dlameter of the arms is varled.

The trend is also similar to that of the conventional folded dipole as seen in chapter 4.0 . The impedance transformation ratio on a meander folded dipole increases as the diameter ratio $\rho_{2} / \rho_{1}$ is is increased.

For diameter ratios smaller than 1.0, the resonant resistance is smaller than the reference resistance of 46 ohms, corresponding to a diameter ratio equal to 1.0 .

There are small changes in the resonant frequency, but hese can again be explained by the fact that a change in wire diameter, entalls a change in geometry, and therefore the electric and magnetic fleld distributions are affected.

From this test, there is insufficient data to try to establish any empirical formula for the calculation of the impedance, or its transformation ratio. However, from the limited results given by MININEC, we can at least draw the conclusion that the resonant imprdance can indeed be adjusted by means of varying the diameter of the conductors.

At this point, we must remember that MININEC results for the impedance of this antenna are probably innaccurate. However, we are interested at this point, only in relative changes in the antenna impedance. Within this context, MININEC confirms the impedanceadaptablitty of the folded meander dipole.

This property, can only be ascertained, of course, within the range of diameter ratios analysed by MININEC: 0.3 to 4.0 . 
Greater, or smaller diameter ratios would have to be analysed by experimental methods, or using the more powerful NEC program.

Also, as was explained in chapter 4.0 , the impedance transformation ratio is also a function of the ratio d $/ \rho_{1}$ In our case, this ratio was quite large, since our wire radil were in the order of millimeters. The distance d between arms is a parameter that in the case of a folded meander dipole is not clearly defined. For the purpose of this analysis, it was defined as the distance between the innermost conductors of the meander dipoles composing the antenna. However, the distance d could also be defined as the distance between any of the other conductors in both arms. In any case, however we decide to define $d$, the ratio d/ $p_{1}$ remains quite large for this particular geometry.

\subsection{CONCLUSIONS:}

A model of the folded meander dipole has been analysed with MININEC and several valid conclusions can be drawn.

First, the antenna impedance behaves much like that of a conventional folded dipole: it has a point of first resonance characterized by a very high input resistance, and a point of second resonance with a more moderate value of resonant resistance.

Also, the impedance, can be assumed to be higher than for a meander (open) dipole.

The antenna bandwidth, is indeed very small, compared to a conventional dipole, and smaller than a meander dipole. As was sald before, this reduction in bandwidth was expected, due to the characteristics of a folded dipole geometry.

The radiation pattern is identical to the folded dipole, or to the meander dipole, for that matter. The gain of the antenna, as given by MININEC, cannot be considered valid, until the impedance values are validated by experimental results.

MININEC has obviously failed to yield numerically accurate results for the impedance of the dipole, as was seen in section 5.3 . due to the program's inherent limitations and to the complexity of the model.

Nevertheless, the impedance transformation ratio property seems to be valid for this type of antenna, and it is reasonable to expect that a design can be accomplished with any suitable impedance.

However, sufficient data has been gathered by this analysis, to atempt to validate the results with an experimental model. The results are encouraging enough to expect that the model will yield a practical reduced-size antenna suitable for narrow bandwidth applications. 


\subsection{EXPERIMENT}

\subsection{MEASURING TECHNIQUES AND EQUIPMENT}

The experiment described in this chapter, was conducted to confirm the results previously obtained by MININEC, and most important, to prove the feasability of the subject design in real-iife conditions. Also, since some of the results given by the program were suspect of being numerically inaccurate, the experiment sought to obtain practical data that would complement the computed results.

The purpose was to build a physical model of the meander folded dipole antenna, and to test this prototype, in the laboratory.

The test were conducted at F.I.U.'s Communication Laboratory. The primary test equipment utilized was the Hewlett-Packard HP 4193A Vector Impedance Meter. This instrument measures impedance magnitude and phase. An internal oscillator provides test signals from $400 \mathrm{KHz}$ to $110.0 \mathrm{MHz}$. This test signal is a constant current between $10 \mu \mathrm{A}$ and $100 \mu \mathrm{A}$, depending on the range of the measured impedance.

The instrument measures and displays impedance magnitude from $10 \mathrm{~m} 11 \mathrm{llohm}$ to $120 \mathrm{~K} 1 \mathrm{lo}-\mathrm{ohm}$. Impedance phase is displayed from -180 degrees to 180 degrees.

The accuracy of the instrument is as good as $3 \%$ of reading, for the magnitude, and 3.28 for the phase. [12]

Measurements are taken with an RF probe, that can be adapted several coaxial connectors. For our experiment, the adapter used was of the BNC type.

One of the major constrainst of this measuring system, is the fact that it is limited to antennas or components that can be fed only with a coaxial line.

As is well known, dipoles and folded dipoles, have to be fed by a balanced transmission line: for instance a 300 ohm parallel line.

A balanced transmission-line is defined as one where the currents on both sides of the line are identical in magnitude, and 180 degrees out of phase. [9] 
Coaxial lines, are obviously not balanced lines, since typically the shield, or outer conductor, of the transmission line is grounded. Coaxial transmission lines are very well sulted for applications where a ground plane exists, such as in the case of monopoles or whip antennas.

When it becomes necessary to feed a dipole antenna with a coaxial transmission line, it is necessary to use a special kind of impedance transformer. These are known as "Baluns", which stands for "balanced-to-unbalanced" transmission line transformer. These devices typically also adapt the antenna impedance to the coaxial line: for example $50 \mathrm{ohms}$ to a $300 \mathrm{ohms}$ input impedance.

However, in our case, where the purpose was to obtain a direct reading of the antenna impedance, it was impractical to distort the readings with the use of any impedance-adapting devices.

This posed a problem, since obviously our antenna required the use of a balanced transmission line.

As a practical solution to this problem, it was decided to make use of an alternate geometry for the meander folded dipole, that would utilize the ground plane, and therefore could be fed by a coaxlal line.

It is known that a ground plane will provide an "image" or reflection of the antenna connected to 1t [4], [6]. Therefore, the geometry of the meander folded dipole described in chapter 5.0 was literally cut in half, with one end being fed by a coaxial adapter and the other was connected to a ground plane. The ground plane would then, by reflection, provide the other half of the antenna.

This test set-up is described in more detail in section 3.3

The antenna impedance, is slightly changed by this method, but the limitations of the test equipment mandated that one such solution be implemented, in order to obtain useful readings.

The ground plane was composed of two aluminum sheets, $0.3 \mathrm{~cm}$. thick, connected with conducting adhesive foil tape. The total dimensions of the ground plae were $2.08 \mathrm{~m}$. by $1.87 \mathrm{~m}$.

It is necessary to point out that, other than impedance measurements, no other test were to be carried out. The lack of a reliable means of measuring the radiated electric and magnetic fields around the antenna, precluded us from attempting to measure other antenna characteristics, such as the radiatolon pattern or the antenna efficiency. 
However, the impedance measurement would provide us with valuable information about the antenna frequency response and bandwidth.

Furthermore, the laboratory results would indeed confirm, or perhaps invalidate the results obtained with MININEC.

One last consideration was the test equipment frequency range. since the highest attainable frequency was $110 \mathrm{MHz}$, all the antenna models tested, had to be resonant at frequencies below this limit.

This did not pose a real problem, but rather provided an advantage. At lower frequencies, all parasitic effects of lead and connector capacitance and inductance are reduced, and it is easier to obtain more accurate readings.

\subsection{VALIDATION}

\section{2 .1 TESTING A MONOPOLE OVER CONDUCTING GROUND PLANE}

The first step in testing a prototype of the meander folded dipole antenna, was to validate the test set-up.

This was again accomplished by testing a known antenna, in this case a thin wire monopole. This antenna was installed in our test stand and connected to the test equipment through a coaxial panel-mount BNC connector mounted in the aluminum sheet forming the ground plane.

The purpose was to compare the practical results of this wellknown antenna, and compare them to existing theoretical and practical data, as well as to MININEC results.

This would give us some information about the validity of our test set-up and the performance of the test-equipment used.

Figure 6.1 shows the test stand with the monopole antenna connected.

Note that the probe is under the ground plane, therefore not interfering in any way with the antenna radiated field.

The monopole was built of thin 22 AWG tinned-copper wire. This gage represents a wire radius of approximately $0.32 \mathrm{~mm}$. The wire was soldered to the center tip of the BNC connector, using standard Tin-Lead electrical solder.

The ground-plane, had the dimensions and characteristics already described in section 6.1 . 
BNC CONNECTOR

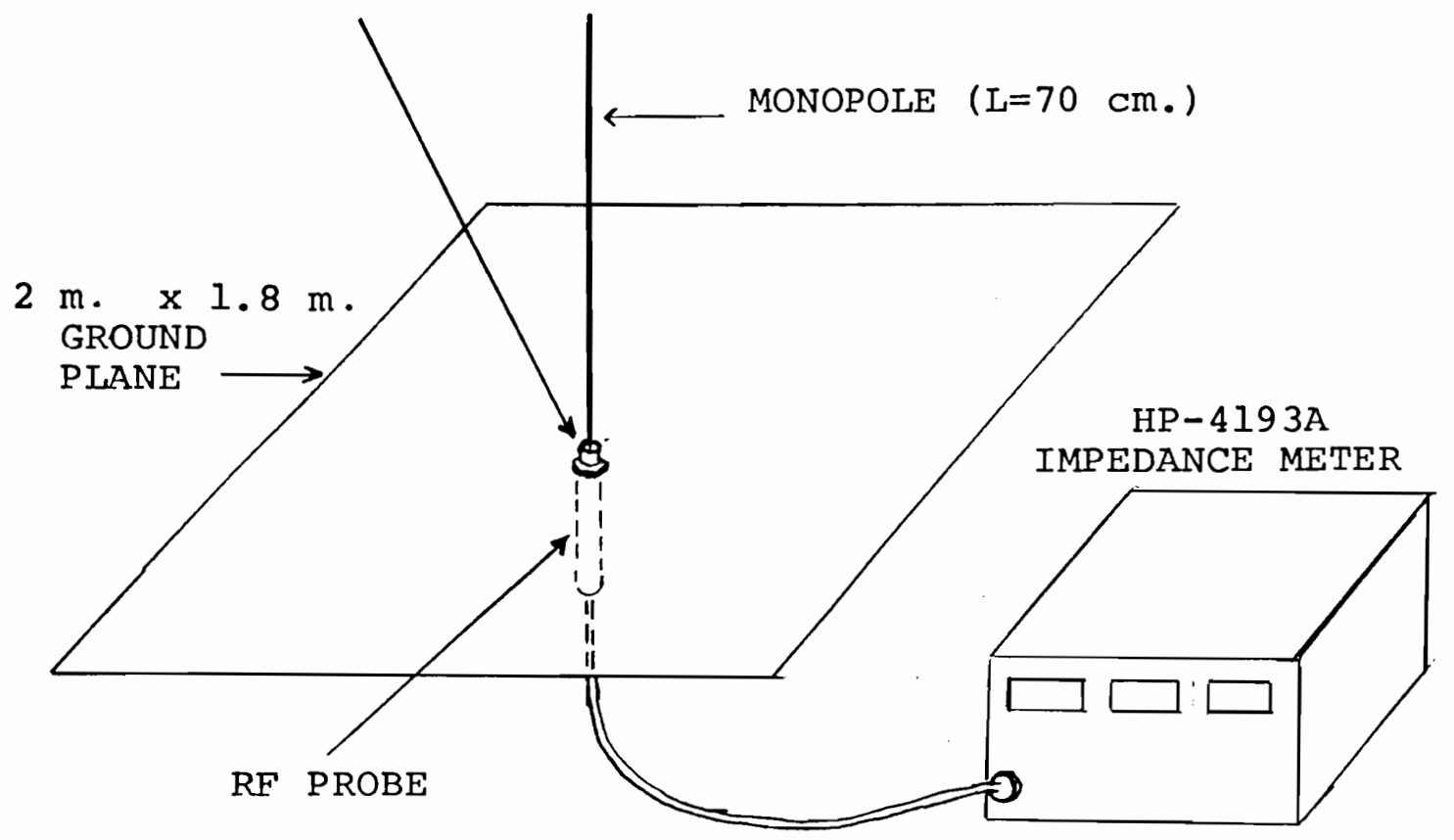

FIGURE 6.1 : TEST SET UP FOR MONOPOLE OVER GROUND PLANE. 
The antenna was mounted on a plece of styrofoam. This material has an electric permittivity equal to that of air [4]. Therefore, it does not affect the measurement system in any way.

The antenna was attached to the styrofoam with plastic adhesive tape, to held it perpendicular to the ground plane.

The antenna was designed to have a resonant frequency around $100 \mathrm{MHz}$, due to the operating considerations explained before. Thus, it was cut to a length of exactly $70 \mathrm{~cm}$. This gives a length-to-diameter ratio of over 100,000 .

A frequency sweep was conducted from $5 \mathrm{MHz}$. to the maximum frequency attainable with the tester of $110 \mathrm{MHz}$.

The results are shown in table 6.1. This table also shows the results obtained with MININEC, for an identical monopole, at the same frequencies.

These results are also shown in graphical form in Figures 6.2 for the resistance, and 6.3 for the reactance of the monopole. Also shown, are the MININEC results, and theoretical data for thin monopoles obtained from [6].

In general, we see good concurrence between the MININEC and data from [6], with the experimental results. There are, however, some significant differences in the values of resistance and reactance, and also there is about a $3 \%$ difference in the resonant frequency.

These differences can be explained by the less-than ideal testing conditions encountered in the laboratory, and they will be discussed in more detail in the section 6.2.2.

\subsubsection{CONSIDERATIONS ON THE EXPERIMENTAL RESULTS}

The most obvious differences encounterd in the previous validation test with the MININEC results, are:

- Differences in the values of resistance and reactances: In general, the reactances and resistances of the experimental results seem to be of a lower numerical value.

- The resonant frequencies differ by about $3 \mathrm{MHz}$, or $3 \%$

These differences can be explained by the fact that MININEC assumes ideal conditions such as perfectiy straight wires, an Infinite ground plane, a constant wire-radius, etc. These conditions can differ significantly from the real-world environment of the laoratory. 


\begin{tabular}{|c|c|c|c|c|}
\hline$F(M H z)$ & Mea & $\begin{array}{l}\text { isured Impedance } \\
\text { (Ohms) }\end{array}$ & $\begin{array}{rr}\text { MININEC } \\
\text { (Oh }\end{array}$ & $\begin{array}{l}\text { Impedance } \\
\text { (ms) }\end{array}$ \\
\hline 5 & 1 & $-\quad j 3220$ & 1 & $-\quad J 5399$ \\
\hline 10 & 1 & $-\quad j 1612$ & 0.2 & $-\quad j 2684$ \\
\hline 15 & I & $-\quad$ J 1070 & 0.4 & $-\quad$ j 1773 \\
\hline 20 & 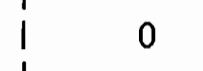 & 795 & 0.8 & $-\quad j 1312$ \\
\hline 25 & 1.1 & $-\quad j$ & 1.3 & $-\quad f 1031$ \\
\hline 30 & 1.0 & $-\quad j$ & 1.9 & $-j 840$ \\
\hline 35 & 0.8 & $-j$ & 2.6 & - J 701 \\
\hline 40 & 0.7 & $-j$ & 3.4 & $-j 593$ \\
\hline 45 & 0.8 & $-j$ & 4.4 & $-\quad j 507$ \\
\hline 50 & 1.4 & $-\quad j$ & 5.5 & $-\quad j 435$ \\
\hline 55 & 1.0 & $-j$ & 6.6 & - J 386 \\
\hline 60 & 0.7 & $-j$ & 8.4 & $-\quad f 321$ \\
\hline 65 & 0.9 & 187 & 10.1 & $-j 274$ \\
\hline 70 & 0.8 & $-j$ & 12.1 & $-\quad j 232$ \\
\hline 75 & 3.0 & $-\quad j$ & 14.5 & - f 192 \\
\hline 80 & 4.8 & $-j$ & 17.1 & - j 156 \\
\hline 85 & 8.9 & $-j$ & 20.1 & $-\quad j 121$ \\
\hline 90 & 14.8 & $-j$ & 23.6 & $-\quad j 87.5$ \\
\hline 95 & 22.3 & $-j$ & 27.6 & - j 55.0 \\
\hline 99 & 29.6 & $-j$ & 31.2 &.- J 29.4 \\
\hline 99.6 & 31.6 & $+j$ & 31.8 & $-\quad$ J 25.6 \\
\hline 103 & 37.4 & +1 & 35.4 & $-j$ \\
\hline 105 & 55.6 & +1 & 8.7 & $+j$ \\
\hline 110 & 85.0 & $+j$ & 43.9 & $+j$ \\
\hline
\end{tabular}




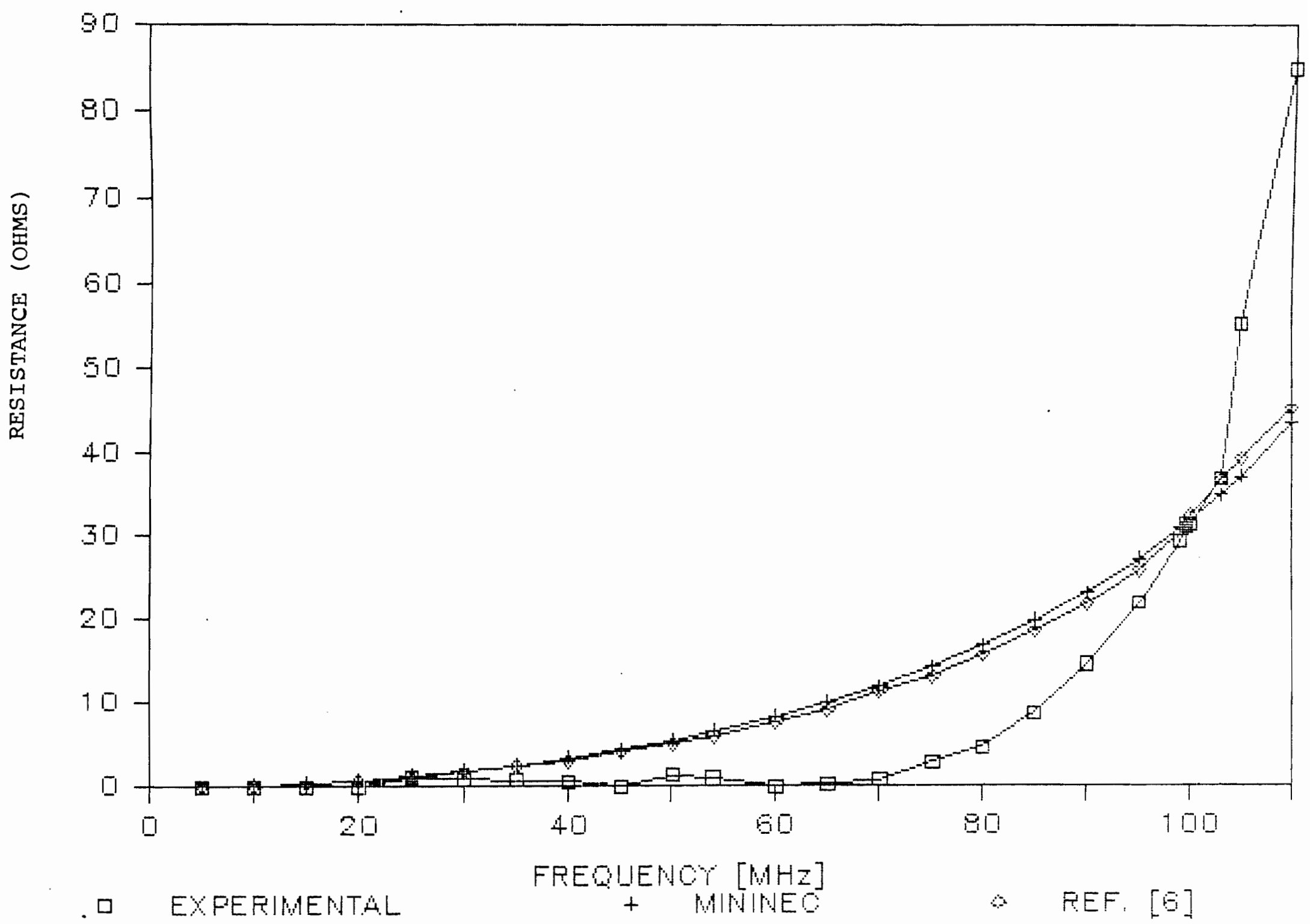

FIGURE 6.2: MEASURED RESISTANCE OF A WIRE MONOPOLE SHOWING MININEC RESULTS AND DATA FROM [6] 


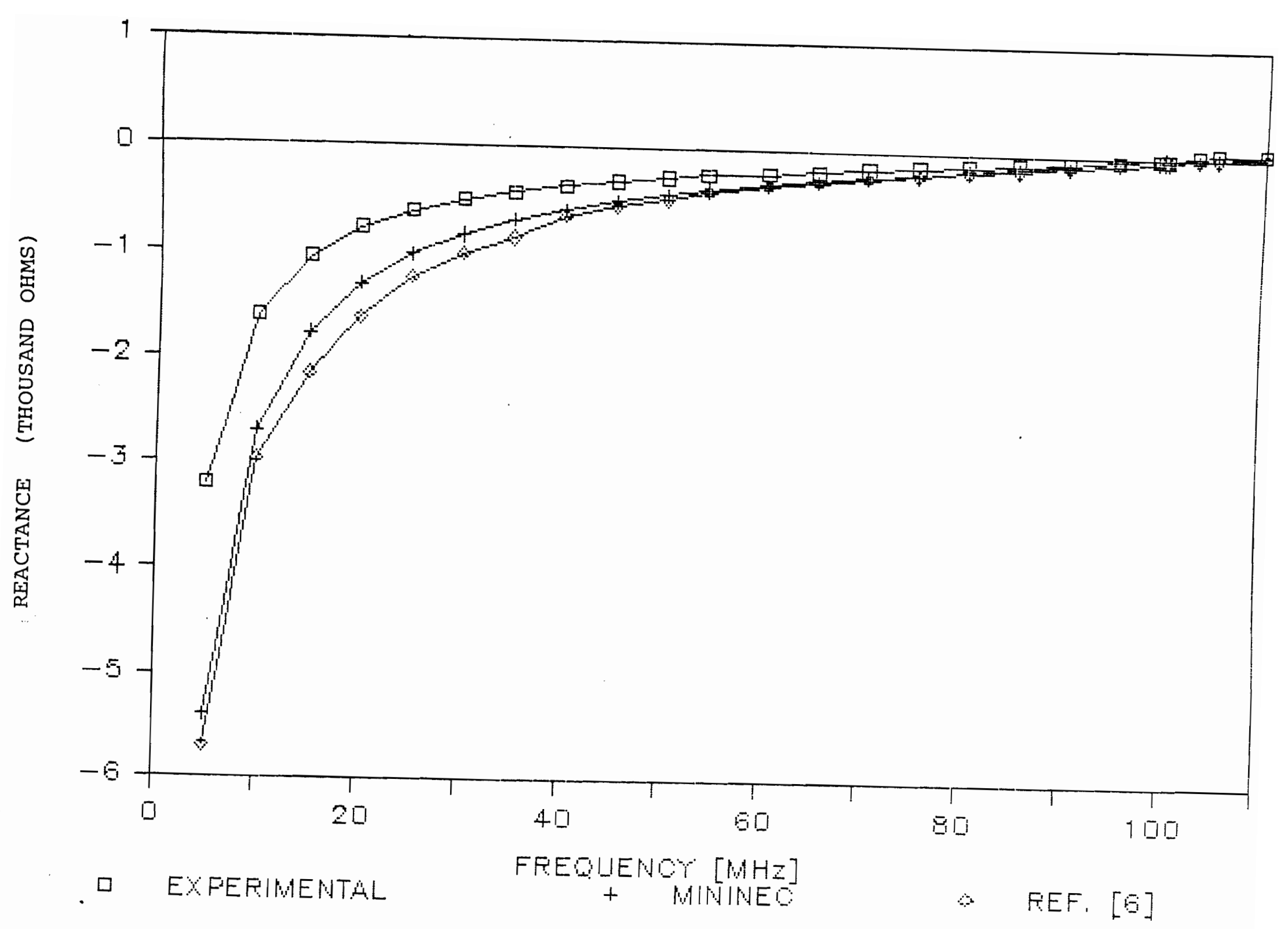

FIGURE 6.3: MEASURED REACTANCE OF A WIRE MONOPOLE SHOWING MININEC RESULTS AND; DATA FROM [6]. 
The most significant drawbacks of the laboratory environment are:

1- The qround plane: MININEC assumes a perfectly conducting, infinite ground plane. In the laboratory, the ground plane was made of Aluminum, which has a finite resistance, and was far from being infinite.

In fact, at the resonant frequency of around $100 \mathrm{MHz}$., the wavelength is 3 meters. Our ground plane measured roughly $2 \mathrm{~m} . \times 2 \mathrm{~m} .$, a ground plane whose larger linear dimension is less than a wavelength. This in fact, will conslderably. alter our results. Unfortunately, a larger ground plane was physically impossible to build in the confined space of the laboratory. Furthermore, a ground plane that would approach the MININEC conditions would have taken several wavelengths in diameter ( 5 to 10 wavelengths). This amounts to 15 meter in diameter!

2- Parasitic capacitance and Inductances: These effects tend to increase with higher frequencies. To quote a few: the HP 4193A tester probe has a specified capacitance of $11 \mathrm{pF}$.[12]. Also, a BNC coaxial connector will have 1 to $2 \mathrm{pF}$ of capacitance. There is also the inductance of the probe cable. In particular, the parasitic capacitances will tend to make the experimental antenna more capacitive, and it will resonate at a lower frequency.

3- Test equipment limitations:

We must remember that the $\mathrm{HP}-4193 \mathrm{~A}$ specifies an accuracy of only $3 \%$ for magnitude and 3.28 for the phase. Any error that falls within this range can be assumed to be within the normal operating limits of the instrument.

4- other factors:

Such as changes in wire radius due to poor manufacturing. small bends in the wire, or the antenna wire not being perfectly perpendicular to ground, all will contribute to make the results differ from those of the computer simulation. Also, there is to consider the effect of soldered connections, poor contact of the probe, ground plane not being perfectly flat, etc. etc.

All of these contribute, in a minor way to make the readings different from the computed results. However, it is very important to know these differences, and to understand the limitations of the laboratory arrangement. This will be useful in understanding the results obtained while testing a model of the meander folded dipole, and to interpret the experimental results accurately. 


\subsection{TEST OF A FOLDED MEANDER DIPOLE.}

\subsubsection{ANTENNA CONSTRUCTION AND TEST SET-UP:}

As was explained previously, the characteristics of the instrument used, precluded the test of a full folded meander dipole model, due to the fact that connection to the tester had to be done through a coaxial cable.

It is a well-known fact that antennas above an infinite conducting ground plane behave as if they there was an image-antenna below the ground plane. This is called the Theory of Images, and is discussed in detail in [4], [6], [8] and [13].

The impedance of an antenna over a conducting ground plane, will be half the impedance of an antenna with twlce the length, suspended in free space. [4] [6].

This property allowed us to use a model of the Meander Folded that was one-half of the geometry described in chapter 5.0 . one end of this prototype was conected to the ground plane, and the other end was fed the test signal througha BNC coaxial cable.

Figure 6.4 shows the actual prototype antenna geometry, and the image antenna below the ground plane.

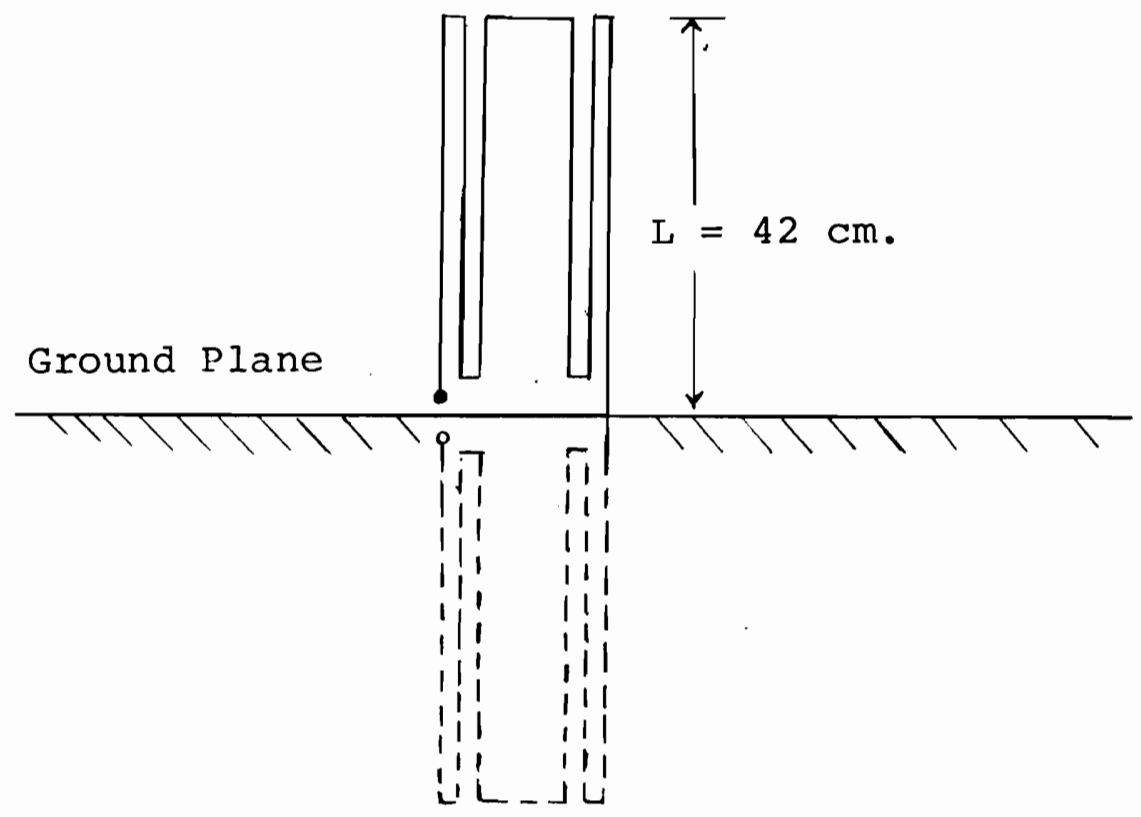

FIGURE 6.4: GEOMETRY OF THE FOLDED MEANDER DIPOLE WITH IMAGE ANTENNA BELOW GROUND PLANE 
The antenna was built very much like like the monopole used for validation. In fact, it was desirable to change as little as possible in the test set-up, so as to be able to have a reliable reference when evaluating the test results.

The wire used was the same 22 AWG tinned-copper wire used for the validation test. Also, the ground plane, had the same dimensions as before.

The antenna was also designed to be resonant at around $100 \mathrm{MHz}$. This frequency was below the maximum frequency attainable with the instrument, and yet was high enough for the dimensions of our prototype antenna to be moderate.

In calculating the heigth of the antenna, we estimated a typical size-reduction factor of 0.55 ( See Chaps. 3.0 and 4.0). At $100 \mathrm{Mhz}$, the wavelength is $3.0 \mathrm{mts}$. in free space. Our folded meander dipole antenna would be therefore resonant at a helgth of 0.55 half-wavelengths, or $83 \mathrm{~cm}$.

Since we were dealing with a model that was half-the size of the geometry under study, our model would have a helgth of:

$$
\mathrm{L}=83 \mathrm{~cm} \cdot / 2=41.5 \mathrm{~cm} \text {. }
$$

This number was actually rounded-up to $42 \mathrm{~cm}$., for ease of construction, and because the reduction factor could easily be as high as 0.6 , making it desirable to have a longer antenna to maintain our resonant frequency of $100 \mathrm{Mhz}$.

The antenna was given a wire separation of $w=1.0 \mathrm{~cm}$, and a distance $d$ between arms (as defined in Fig. 5.4) of $5 \mathrm{~cm}$.

The model was mounted on a piece of styrofoam, which is non-magnetic and has an electric permittivity equal to that of air [4]. This ensures that it would have no effect on the test readings.

The antenna was soldered to the center point of the BNC panelmount connector installed in the ground plane.

The other end of the antenna was connected to ground with a piece of adhesive foil tape (conducting).

This ground connection involved about $15 \mathrm{~cm}$. of wire connected to the ground plane, with the conducting tape.

Figure 6.5 describes the test set-up for the folded meander dipole antenna.

The instrument's RF probe was connected to the antenna through a male BNC adapter. Since the probe and its cable were under the ground plane, it was assumed that they had minimal effect with the measurement. 
1/2 FOLDED MEANDER DIPOLE.

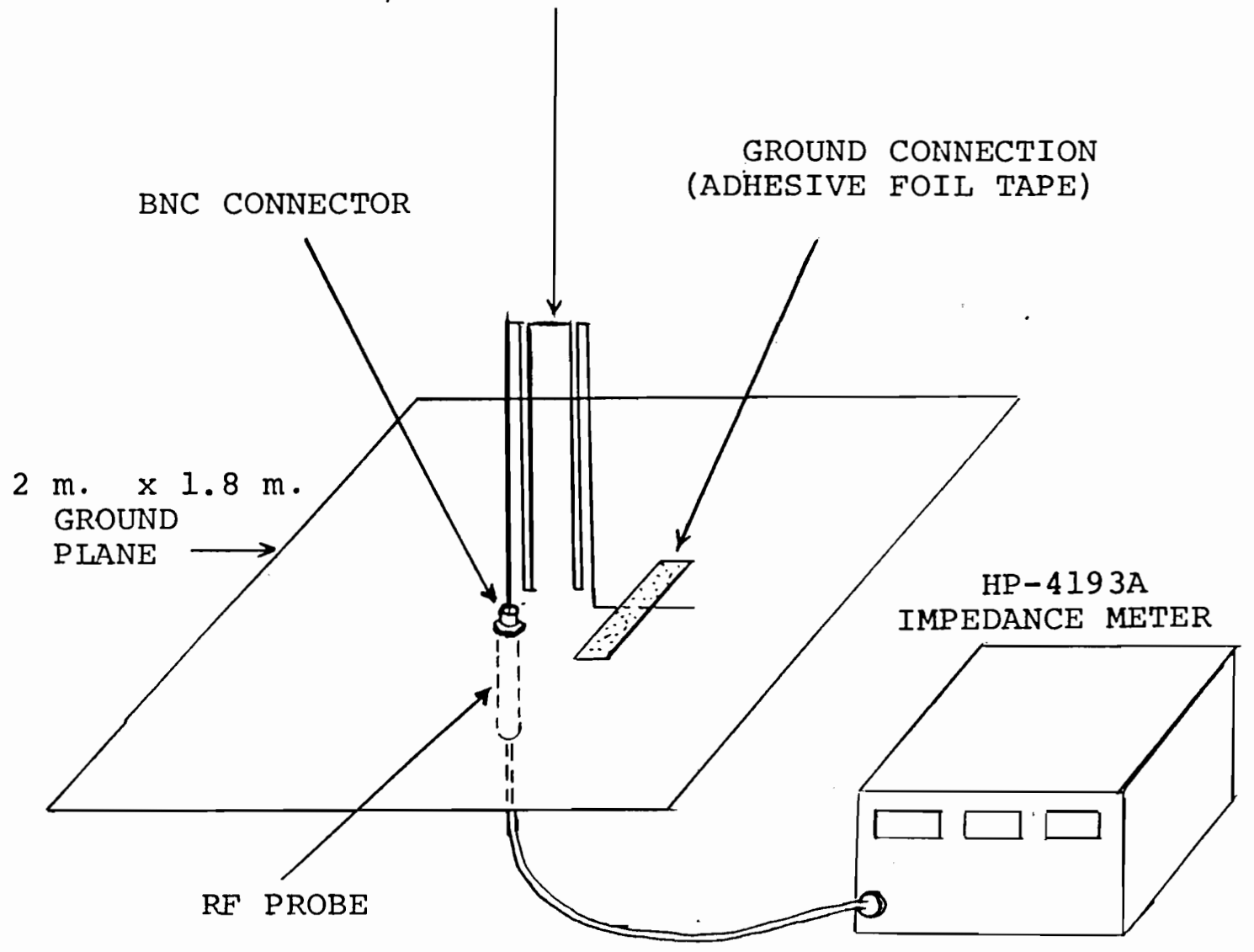

FIGURE 6.5: TEST SET-UP FOR FOLDED MEANDER MONOPOLE 


\subsection{IMPEDANCE MEASUREMENT:}

A frequency sweep was then done through the operating range of the $\mathrm{HF}-419 \mathrm{~g}$ tester, and impedance measuremente were taken.

The results are shown in Table 6.2. They have are also shown in graphical form in Figures 6.6 . and 6.7 .

The experimental readings were in accordance with the expected values. The resonant frequency was $94.6 \mathrm{MHz}$., within 58 of the expected value of $100 \mathrm{MHz}$. ( refer to 6.3 .1 )

At $94.6 \mathrm{Mhz}$, the approximate resonant length L of a monopole over conducting ground is calculated by:

$$
L(\text { monopole })=0.25 \times 300 / 94.6 \times 0.95=75.3 \mathrm{~cm} .
$$

where 0.95 is the size factor described in 4.3 .2 .

Therefore, the measured size-reduction factor $r$ of our antenna is the ratio of the length $L$ (meander) of our prototype antenna, to the length $L$ (monopole) calculated above.

(Both antennas are resonant to $94.6 \mathrm{MHz}$. )

$$
\begin{aligned}
r & =\mathrm{L} \text { (meander) } / \mathrm{L} \text { (monopole) }=42 \mathrm{~cm} . / 73.5 \mathrm{~cm} . \\
\text { or } r & =0.557
\end{aligned}
$$

This value is within 28 to the assumed factor of 0.55 , used for designing the antenna.

The resistance and reactance behave very much as predicted by MININEC for this type of antenna ( Refer to Figures 5.5 and 5.6 )

The impedance is characterized by a point of first resonance with a very sharp change in reactance and a very high resistance. The second resonance also shows a somewhat rapld change in reactance and a more moderate resistance value.

The value of resonant resistance is 32.5 ohms. Th1s is somewhat larger value than expected. This result represents half the value of a meander folded dipole impedance in free space. Which means that the meander folded dipole should have a resonant resistance of :

$$
\text { Ro }=2 \times 32.5 \text { Ohms }=65 \text { Ohms. }
$$

The rapid change in reactance is indicative of a narrow bandwidth around second resonance. This will be discussed in the next section. 
TABLE 6.2: MEASURED FOLDED MEANDER MONOPOLE IMPEDANCE.

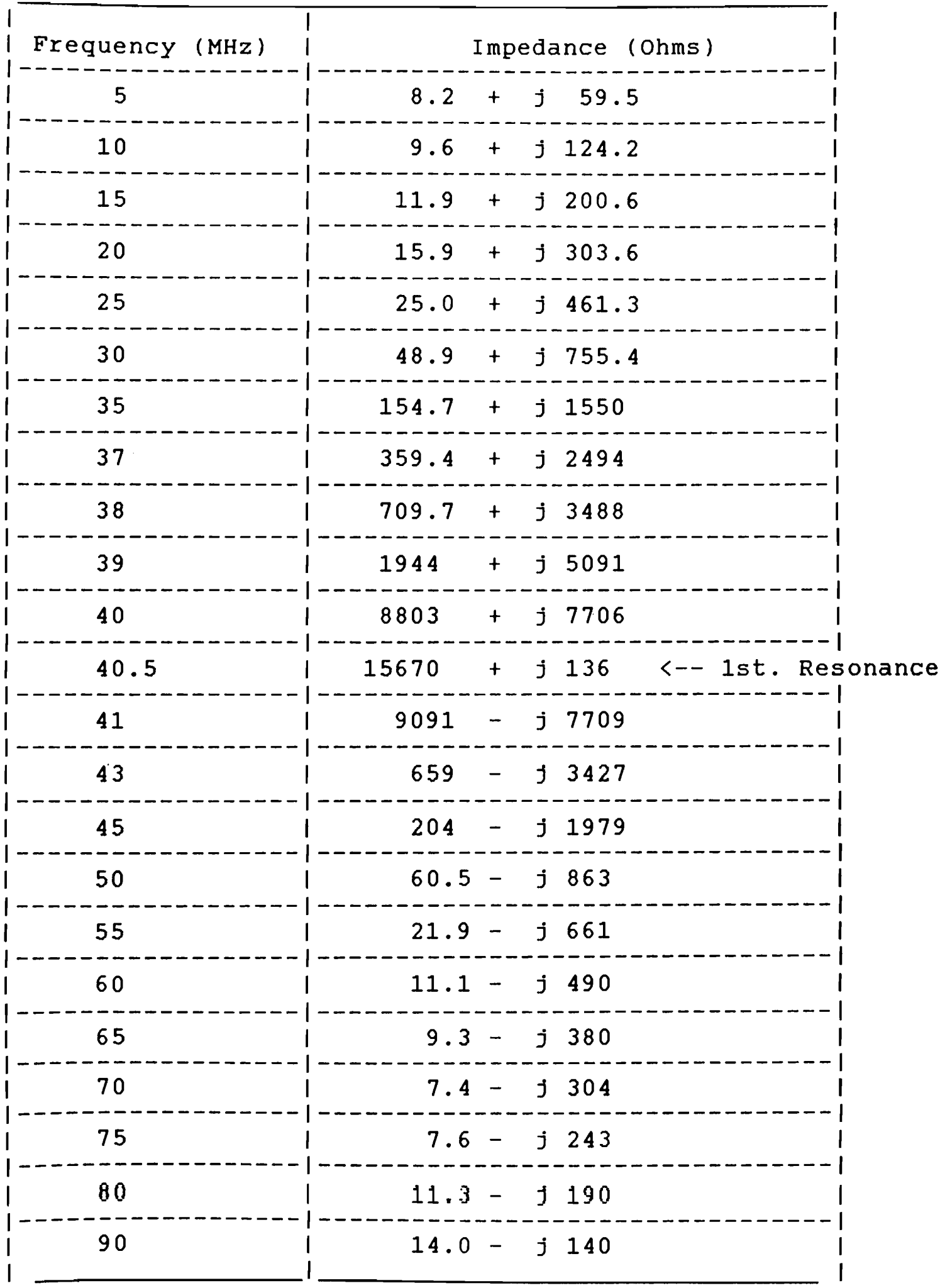




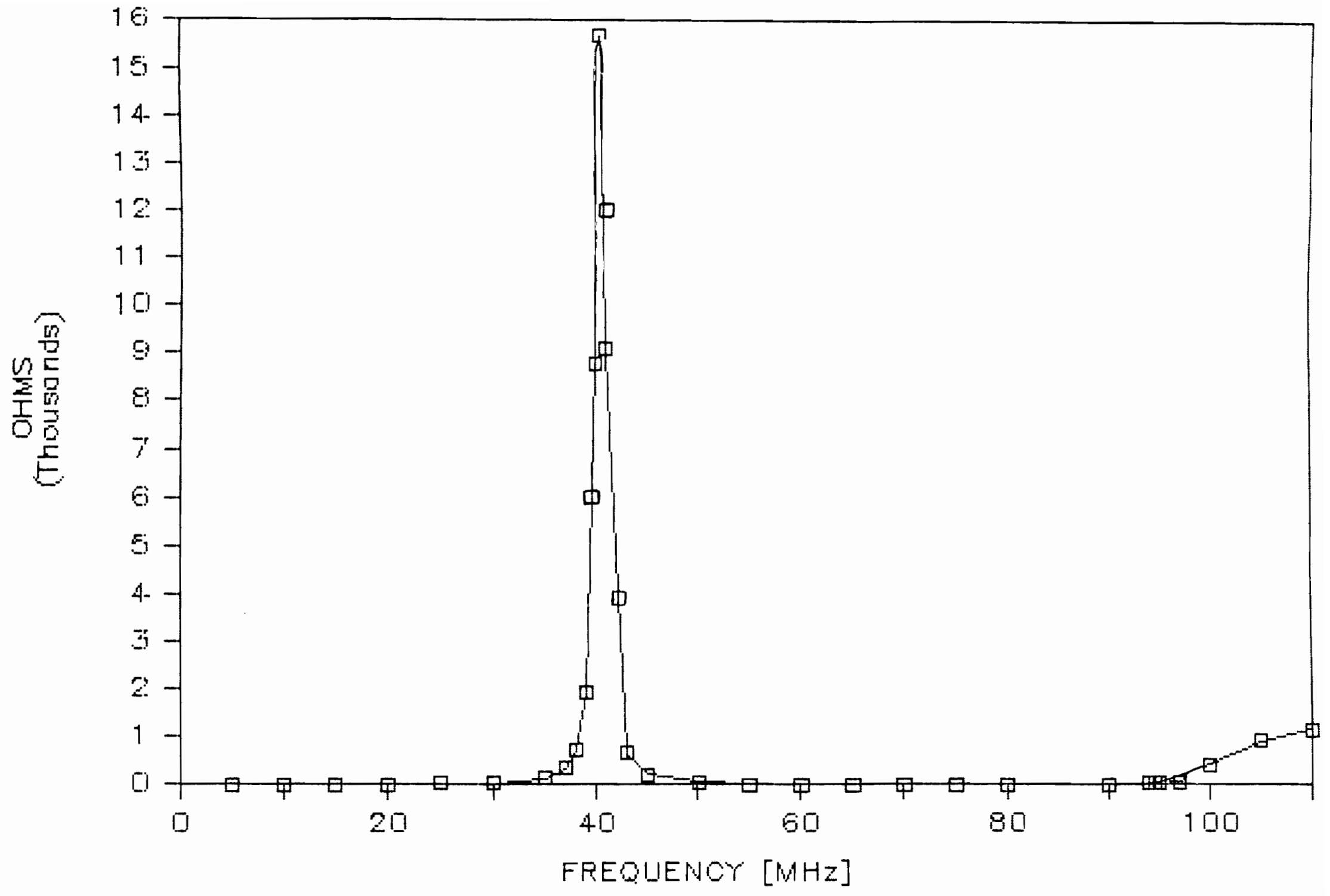

FIGURE 6.6 : MEASURED RESISTANCE OF A FOLDED MEANDER MONOPOLE 


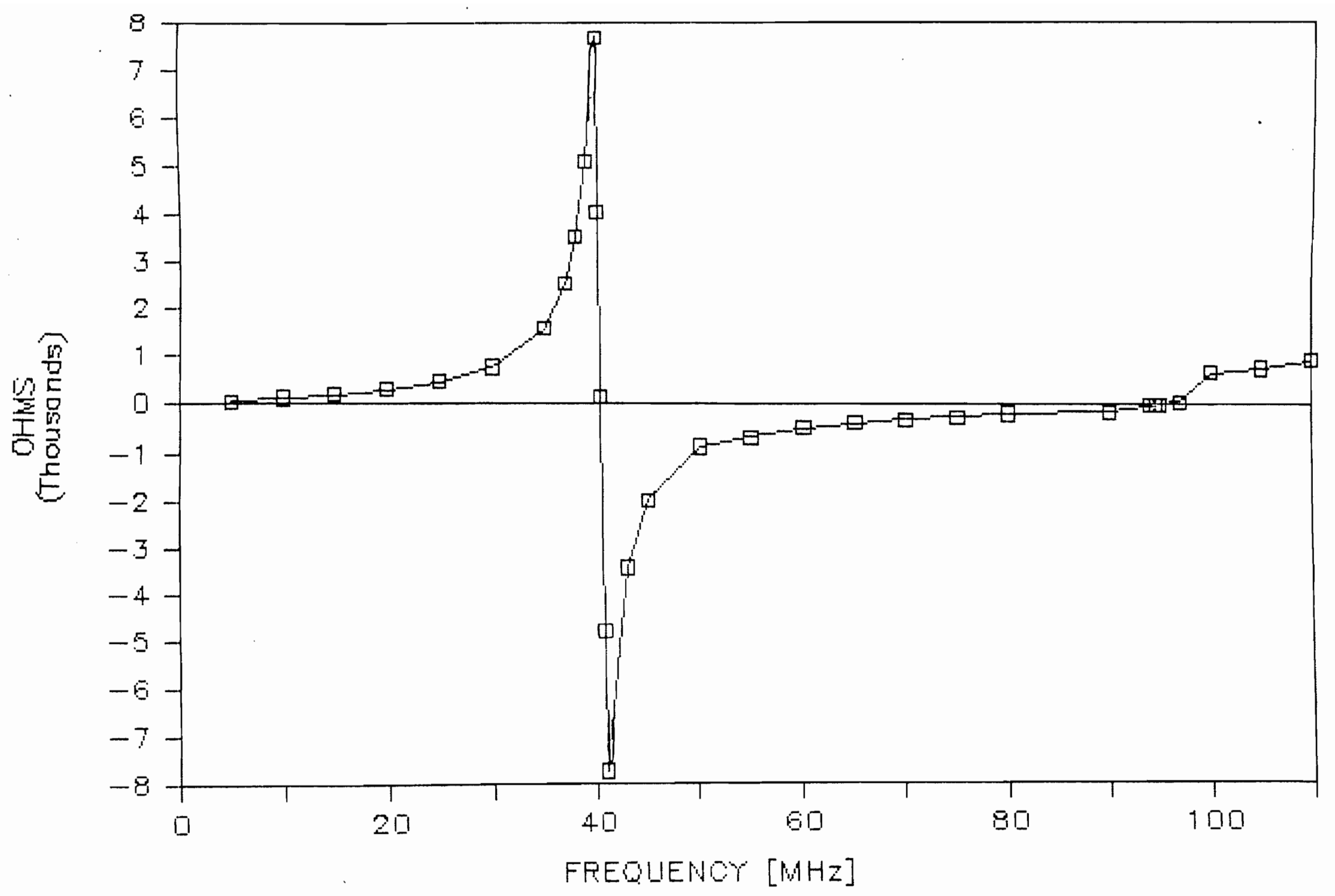

FIGURE 6.7: MEASURED REACTANCE OF A FOLDED MEANDER MONOPOLE. 
TABLE 6.2 (CONTINUED): MEASURED IMPEDANCE OF A MEANDER FOLDED MONOPOLE.

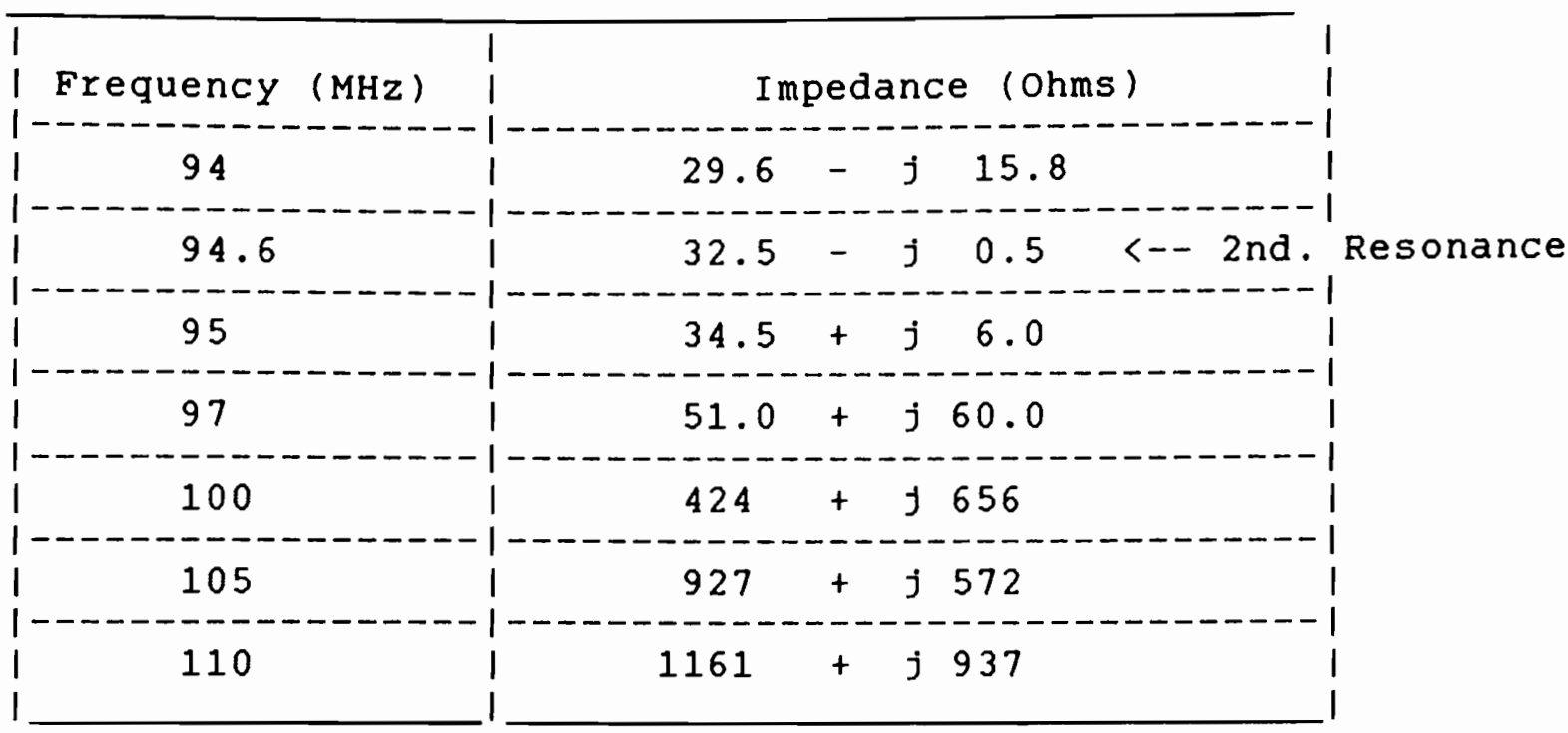

It must be noted that the impedances of table 6.2 are not the direct readings from the experiment, since the instrument only gives magnitude and phase of the impedance. But with these two parameters, resistance and reactance can be easily calculated.

\subsubsection{BANDWIDTH OF FOLDED MEANDER DIPOLE}

The next part of the experiment consisted in measuring the operational bandwidth of the antenna. This was accomplished by taking a new set of reading, around the second resonant frequency. This time, the frequency steps would be smaller in order to obtain a higher frequency resolution.

The antenna tested, was of course, the some one described in the previous section.

The results are shown in Table 6.3 and are also presented in graphical form in the smith-Chart of Figure 6.8 .

Note that the results of Table 6.3 differ slightly from those of Table 6.2. The differences are due to the accuracy of the instrument, which was used in another frequency scale, and to numerical approximations when calculating the impedance values from the Magnitude and phase numbers given by the tester. 
TABLE 6.3: MEASURED IMPEDANCE OF FOLDED MEANDER MONOPOLE AROUND 2nd. RESONANCE.

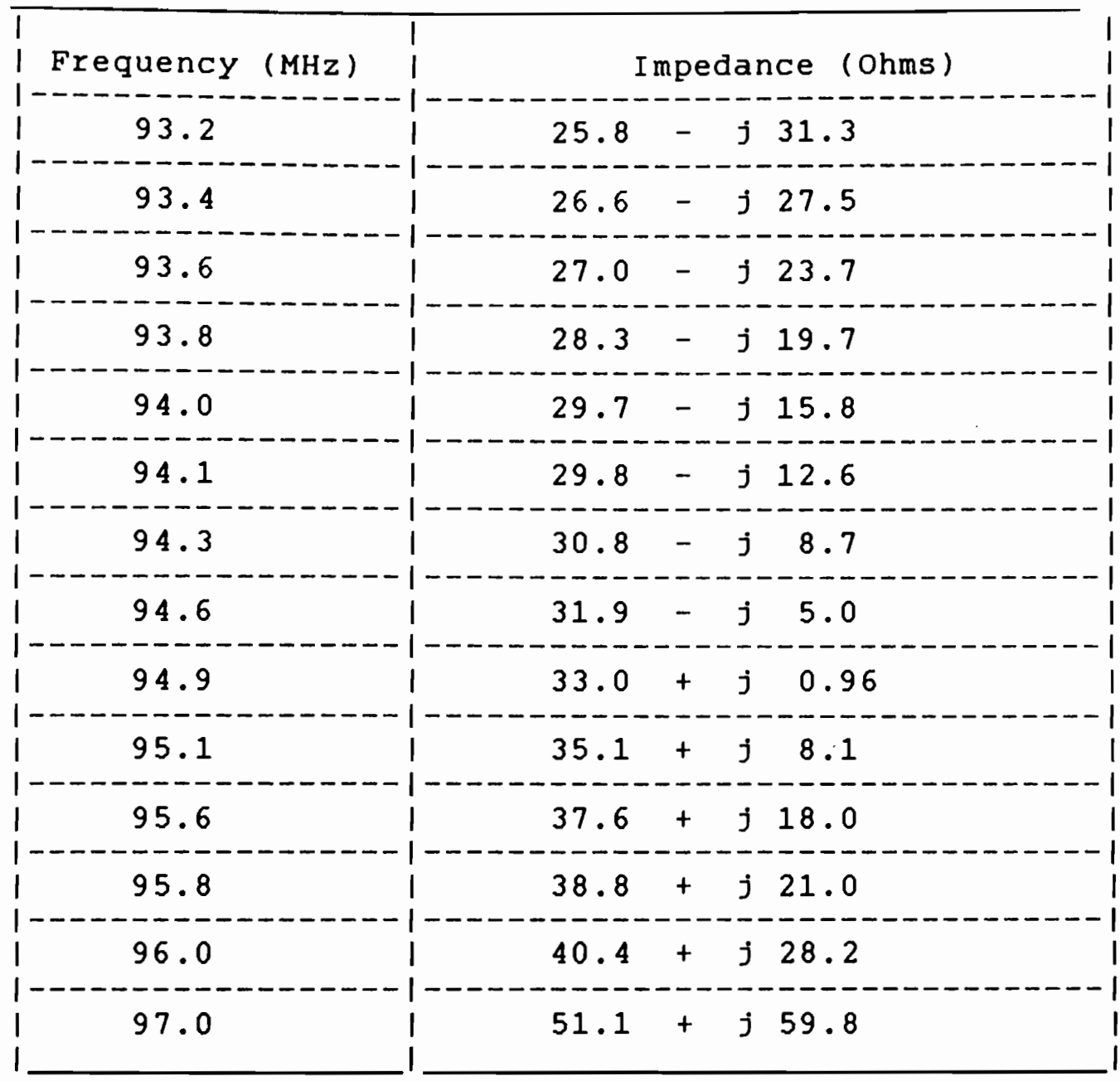

These results have been presented in graphical form in the Impedance Chart of Figure 6.8. The Sm1th chart shown in this Figure also serves to estimate the antenna bandwidth.

So far, we have defined The antenna bandwidth on the basls of a VSWR of 2.0 . This is a very stringent requirement, and very few commercially available antennas can meet this specification through their entire operation range.

A more practical way of defining the bandwidth would be on the basis of a VSWR of 3.0 or higher.

Figure 6.8 shows two VSWR circles, which specify different operating bandwidths for the antenna. 


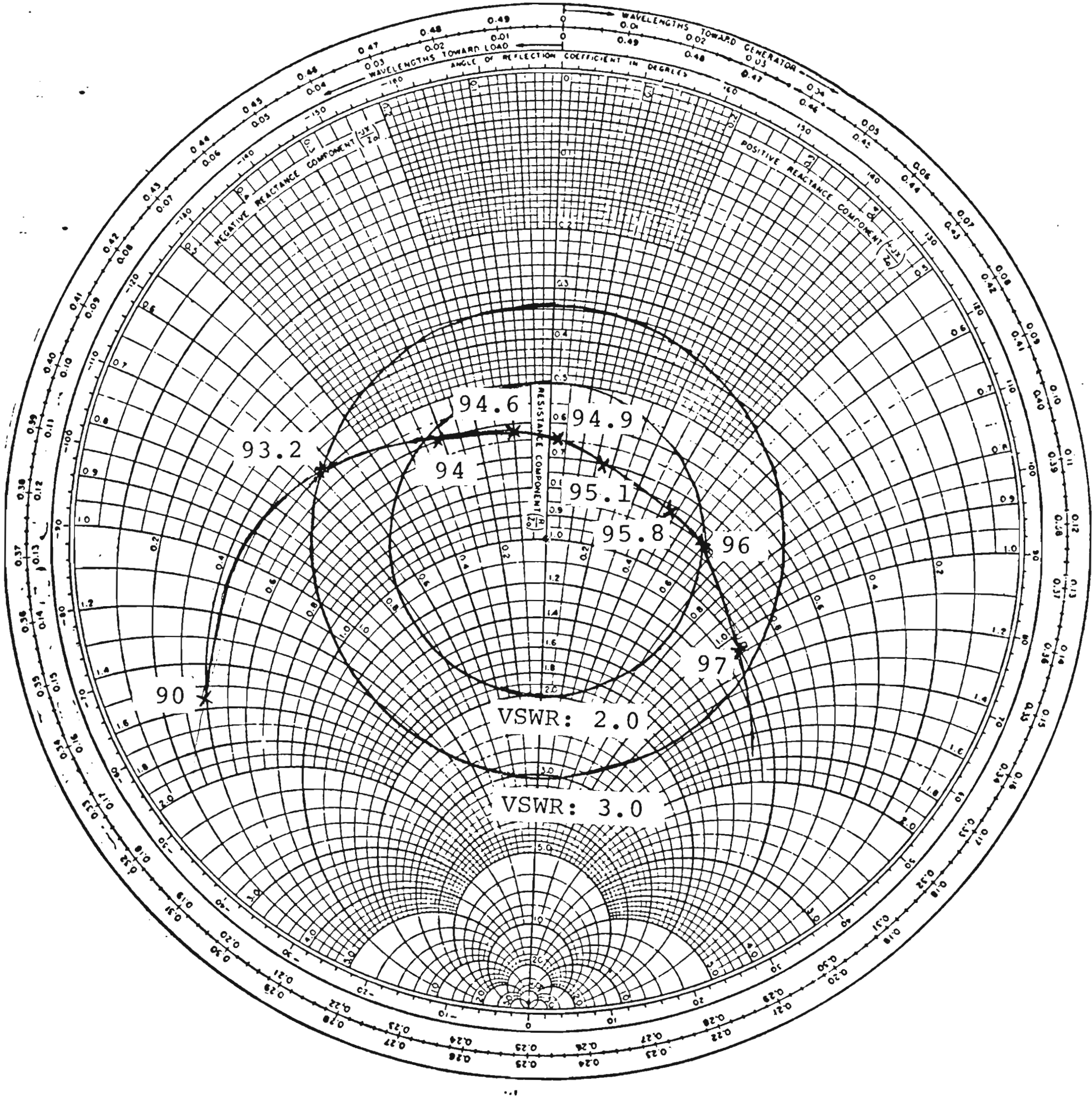

FIGURE 6.8: IMPEDANCE OF FOLDED MEANDER DIPOLE NORMALIZED TO 50 OHMS. 
We can see that for a VSWR of 2.0 , the bandwidth is approximately $2.0 \mathrm{MHz}$. or 2.1 of the resonant frequency.

For a VSWR of 3.0 , the bandwidth is approximately $4.0 \mathrm{MHz}$, or 4.2 of the resonant frequency.

These numbers are higher than those predicted by MININEC in section 5.4.1, where the bandwidth given by the program for different wire separations were all under 1.0 of of the resonant frequency. These practical results tell us our antenna is actually better than predicted by the program, since a wider bandwidth is always desirable.

\subsubsection{EFFECT OF WIRE SEPARATION ON ANTENNA CHARACTERISTICS:}

The wire separation was changed in the antenna used for the previous tests. We have seen in chapter 5.0 that changes in w will somewhat affect the antenna characteristics. The most important effects with increasing $w$ are:

- Resonant Resistance is reduced.

- Bandwidth is reduced.

- Reduction factor is reduced.

The antenna tested previously was modified to have different wire separations of $w=1.0,2.0$ and 3.0 . The separation between arms was kept constant at $5 \mathrm{~cm}$.

Again, a frequency sweep was accomplished around the point of second resonance, for each antenna.

The results are shown in Table 6.4 .

TABLE 6.4: EFFECT OF WIRE SEPARATION ON EXPERIMENTAL ANTENNA CHARACTERISTICS .

(Bandwidth measured for VSWR $=2.0$ )

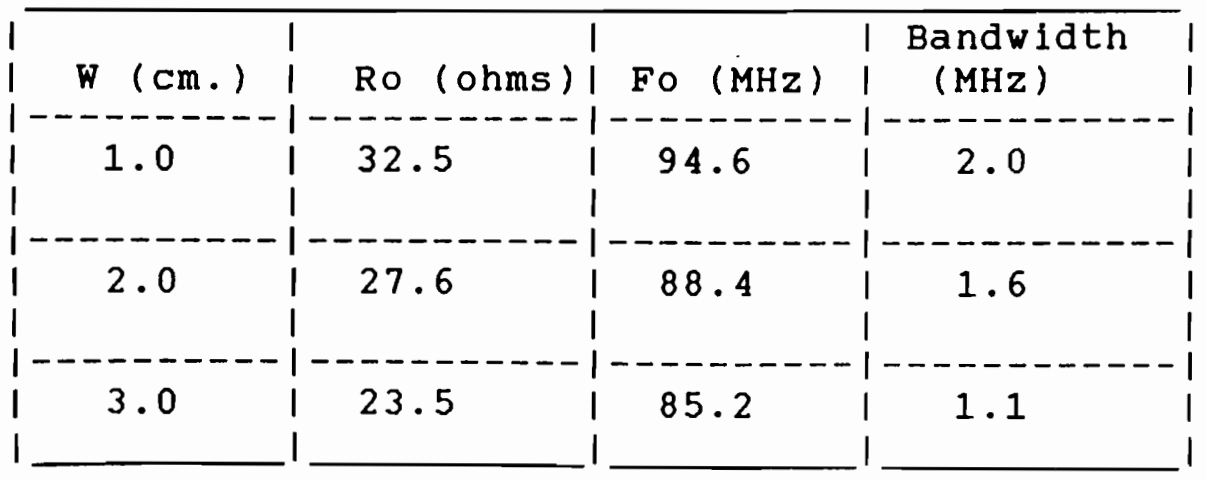


Note that the bandwidth was estimated on the basis of a VSWR $=2.0$

From the results of Table 6.4, we see that the known behaviour of meander antennas as the wire separation is changed [2], is also valid for our experimental model of a folded meander dipole. Note that a wire separation of $w=2.0 \mathrm{~cm}$. would be best suited for working with a 50 ohm transmission line. For $w=2.0 \mathrm{~cm}$, our full-sized meander dipole in free space, would theoretically have a resonant resistance of twice that of the test antenna or Ro $=27.6 \times 2=55.2 \mathrm{Ohm}$.

The experimental results confirm the trend of a lower resonant resistance, and a smaller bandwidth as $w$ is increased. Also, the lowering of the resonant frequency is indicative of a lower reduction factor (more size reduction).

\subsubsection{IMPEDANCE TRANSFORMATION WITH CHANGES IN WIRE DIAMETER.}

The last test accomplished with our experimental antenna was $t$ impedance adaptability property, through changes in conductor diameter in both arms.

Let us remember that our basic antenna was built with a conductor of radius $=0.32 \mathrm{~mm}$..

The first step consisted of changing one arm of our folded monopole with 18 AWG copper wire, which has radius of approximately $0.66 \mathrm{~mm}$.

By connecting the excitation source to one arm and grounding the opposite arm of this antenna alternately, two sets of readings were taken. One corresponds to a radius ratio of:

$$
\rho_{2} / \rho_{1}=0.32 \mathrm{~mm} / 0.66 \mathrm{~mm} \cdot=0.485
$$

and the other corresponding to a ratio:

$$
\rho_{2} / \rho_{1} \quad=0.66 \mathrm{~mm} . / 0.32 \mathrm{~mm} .=2.06
$$

Impedance readings were then taken from this new antenna, using the same test set-up described previously.

The next step was to replace the 22 AWG wire (radius $=0.32 \mathrm{~mm}$.) with a thinner 28 AWG wire, that has a radius of $0.25 \mathrm{~mm}$.

Again, by alternately connecting the instrument to one end of this antenna, and grounding the other, two sets of readings were taken for diameter ratios $p_{2} / p_{1}=2.64$ and 0.38 . 
The results are presented in Table 6.5:

TABLE 6.5: EFFECT OF CHANGES IN CONDUCTOR DIAMETER ON ANTENNA IMPEDANCE.

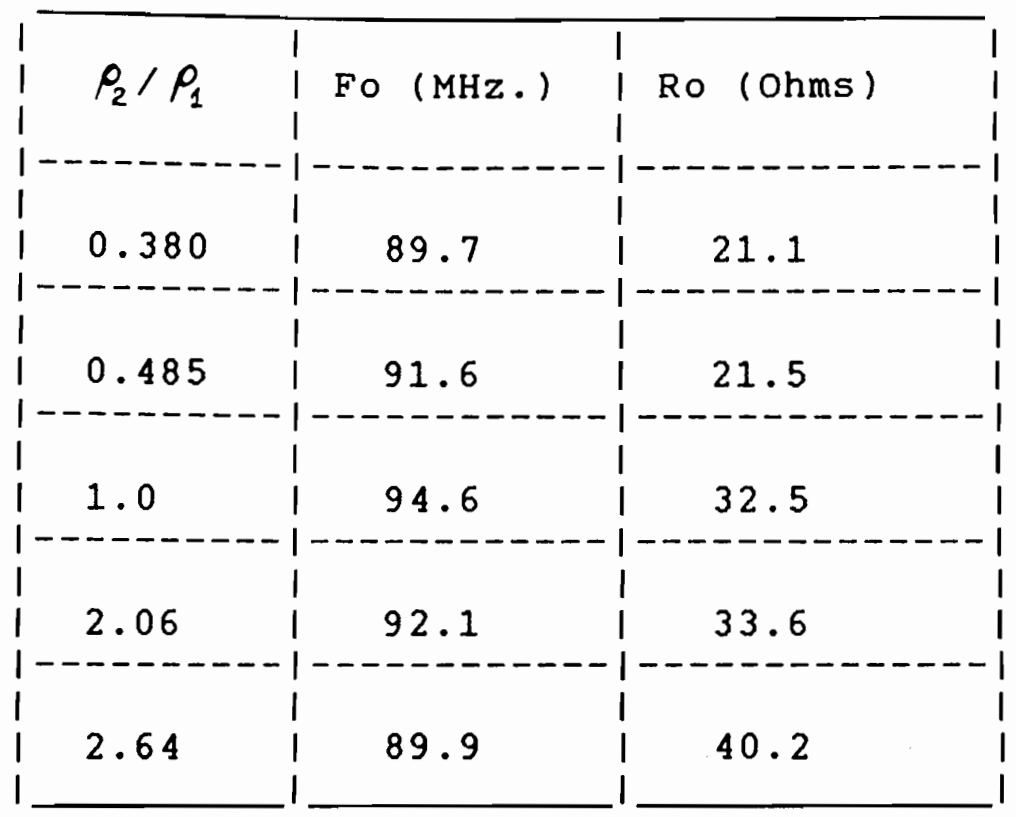

As we can see from the results shown in Table 6.5 , there are indeed changes in the resonant resistance as the conductor diameter is changed. The trend corresponds to that predicted by MININEC, and also to the behaviour expected of conventional folded dipoles.

The changes in resistance are however, quite moderate. We see that the impedance does not increase as drastically as predicted by MININEC in Table 5.9 .

We can also point out that the increase in wire diameters were limited to those allowed by existing type of wires. For higher diameter ratios, copper tubing would have to be utilized. This presents the problem that if the radius of a conductor is comparable to the wire separation, then the geometry of the antenna is altered excessively.

The obvious solution is to build a larger antenna, with a wire separation w that would be much greater than the copper tubing diameter. At this point, as was explained before, we would need a much larger ground plane. This was impractical, if not impossible, due to the dimensions of the laboratory. 
Also note that there are changes in the resonant resistance. This is due to minor differences introduced in the test antennas, during their manual fabrication, and to the fact that the antenna geometry is also slightly altered when the wire diameter is changed.

6.4 DISCUSSION OF EXPERIMENTAL RESULTS.

We have bullt a model of the Folded Meander Dipole and tested it under laboratory conditions.

The results, as presented here are quite encouraging. The antenna has an impedance that can be adapted to several transmission lines. ( 50 Ohms, 75 ohm transmission lines)

The antenna has a working bandwidth of $4 *$ of the resonant frequency, for a VSWR of 3.0. This bandwidth is very much in the same range as many commercially available antennas.

Furthermore, we have investigated two ways by whlch the antenna impedance could be adapted to other transmission lines:

- By changing the wire separation w (Th1s will reduce Bandwidth)

- By changing the wire diameters ratio.

Also, the laboratory experiment has shown that this type of antenna could be built as a full dipole, or, as in the test model, as a monopole with one end grounded.

This last geometry is probably more attractive, since it can be fed with a conventional coaxial transmission line.

The experimental results for the resonant frequency and reduction factor are close to the ones predicted by MININEC within 2 to $3 \%$.

Although the impedance values varied with reference with MININEC, the general behaviour of the antenna was adequately predicted by the program. Let us remember that the resonant resistance value given by the program, in chapter 5.0 was not considered an accurate result, due to the program's limitations.

Although there were no means avallable in the laboratory to measure other important characteristics, such as efficiency and radiation pattern, the results obtalned glve us the confldence that MININEC predictions in refrence to these characteristics will not be significantly different than the real ones for this antenna. 


\subsection{CONCLUSIONS}

\subsection{BASIC THEORY OF MEANDER ANTENNAS:}

Meander antennas exhibit many of the basic characteristics of conventional dipoles and monopoles. We have seen in previous chapters that their radiation pattern is similar to the pattern of these conventional antennas, and we know from [2] that the efficiency is comparable, if somewhat smaller.

The most obvious disadvantage of meander antennas is their low input impedance at resonance, as was seen in chapter 4.0 .

The following sections will attempt to explain some of these characteristics, based upon known antenna theory.

\section{1 .1 SELF AND MUTUAL IMPEDANCE OF ANTENNAS}

Antennas such as dipoles suspended in free space, have an input impedance, that under ideal conditions is not affected by any surrounding structure or nearby conductors.

This ideal input impedance is generally known as the antenna self-impedance. This is the input impedance of the antenna, when its current distribution and radiated fleld are not affected by any surrounding conducting structures.

In an array of antennas, the driving point impedance of an individual element may differ considerably from its self impedance because of the effect of mutual coupling with other elements of the array [6].

In a multielement array, the relations between the currents and voltages are given by:

$$
\begin{aligned}
& V_{1}=I_{1} Z_{11}+I_{2} z_{12}+\ldots+I_{n} z_{1 n} \\
& V_{2}=I_{1} Z_{12}+I_{2} Z_{22}+\ldots+I_{n} Z_{2 n} \\
& V_{n}=I_{n} Z_{1 n}+I_{2} z_{2 n}+\ldots+I_{n} Z_{n n}
\end{aligned}
$$


Where: $\quad V_{n}=$ Impressed voltage at nth. element

In $=$ Current flowing in nth. element

$\mathrm{Znn}=$ Self Impedance of $\mathrm{nth}$. element

$\mathrm{Zmn}=\mathrm{Znm}=$ mutual impedance between mth and $\mathrm{nth}$ element.

The driving point impedance for elementh 1 , for instance, is found from the ratio of the impressed voltage to the current, and is obtained from the above equations as follows [6]:

$$
Z_{1} \text { (input) }=V_{1} / I_{1}=Z_{11}+\left(I_{2} / I_{1}\right) Z_{12}+\ldots+\left(I_{n} / I_{1}\right) Z_{1 n}
$$

It is readily seen that the input impedance or the driving point impedance of a particular element is not only a function of its own self impedance but also a function of:

-The the relative current flowing in the other elements and -The mutual impedance between elements.

Several authors have treated the subject of estimating the value of the mutual impedance between elements [4], [6], [13] In particular, C. Balanis [13] gives simple mathematical relations for estimating the mutual impedance of wires positioned for mutual coupling.

Refering to figure 7.1, showing two wires suspended in space and parallel to each other, the induced voltage at antenna 2 , referred to its current at the input terminal, due to radiation from antenna 1 , is given by:

$$
V_{21}=-\frac{1}{I_{2 i}} \int_{-I_{2} / 2}^{I_{2} / 2} E_{z 21}(z) I_{2}(z) d z
$$

where Ez $z_{21}$ is the E-field component radiated by antenna 1 which is paraliel to antenna 2. It would be calculated as if antenna 2 were absent. I $(z)$ represents the current distribution along antenna 2. This basic relation is used to develop a formula for the mutual impedance between antennas 1 and 2 , using known relations for the E-field and assuming a sinusoidal current distribution in the dipoles. [13]

(7.3) can rewritten as:

$$
\begin{aligned}
V_{21}= & j \frac{\eta I_{1 m} I_{2 m}}{4 \pi I_{2 i}} \int_{-I_{2} / 2}^{+l_{2} / 2} \sin \left[k\left(\frac{l_{2}}{2}-|z|\right)\right] \\
& \times\left[\frac{e^{-j k R_{1}}}{R_{1}}+\frac{e^{-j k R_{2}}}{R_{2}}-2 \cos \left(\frac{k l_{1}}{2}\right) \frac{e^{-j k r}}{r}\right] d z
\end{aligned}
$$




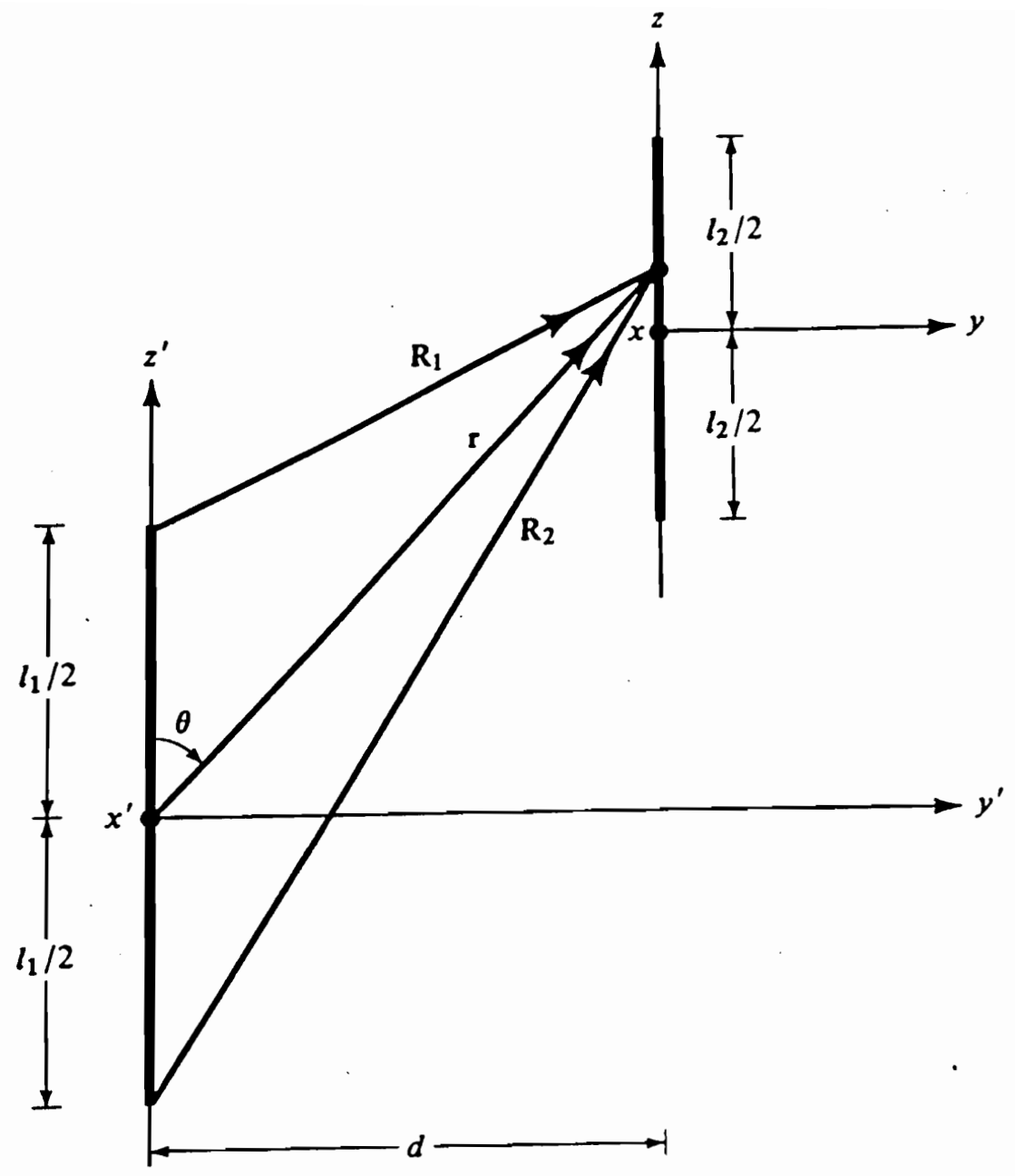

FIGURE 7.1: DIPOLE POSITIONING FOR MUTUAL COUPLING [13]

And the mutual impedance of (7.2), referred to the input current $I_{1 i}$ of antenna 1, can be written as [13]:

$$
\begin{aligned}
Z_{21 i}= & \frac{V_{21}}{I_{1 i}}=j \frac{\eta I_{1 m} I_{2 m}}{4 \pi I_{1 i} I_{2 i}} \int_{-l_{2} / 2}^{l_{2} / 2} \sin \left[k\left(\frac{l_{2}}{2}-|z|\right)\right] \\
& \times\left[\frac{e^{-j k R_{1}}}{R_{1}}+\frac{e^{-j k R_{2}}}{R_{2}}-2 \cos \left(k \frac{l_{1}}{2}\right) \frac{e^{-j k r}}{r}\right] d z
\end{aligned}
$$

where $R_{1}, R_{2}$ and $r$ are the distances of flgure 7.1 
and $k^{2}=\omega^{2} \mu \varepsilon$

$$
\begin{aligned}
& \eta=1 \text { intrinsic impedance of the medium }=\sqrt{\mu / \varepsilon} \\
& I m, I m=\text { maximum currents of antennas } 1 \text { and } 2 \\
& I 1 ; I 1=\text { Input currents of antennas } 1 \text { and } 2
\end{aligned}
$$

The mutual impedance, as given by (7.5) is referred to the current at the input terminals and can be translated to the current maxima by [13]:

$$
Z_{21 m}=Z_{21 i} \frac{I_{1 i} I_{2 i}}{I_{1 m} I_{2 m}}
$$

$$
\text { or } \begin{aligned}
Z_{21 m}= & j \frac{\eta}{4 \pi} \int_{-l_{2} / 2}^{l_{2} / 2} \sin \left[k\left(\frac{l_{2}}{2}-|z|\right)\right] \\
& \times\left[\frac{e^{-j k R_{1}}}{R_{1}}+\frac{e^{-j k R_{2}}}{R_{2}}-2 \cos \left(k \frac{l_{1}}{2}\right) \frac{e^{-j k r}}{r}\right] d z
\end{aligned}
$$

For two identical antennas (each of length $1=n \lambda / 2, n=1,2,3 \ldots$ ) (7.7) can be written in a simplified form. For the case where the two dipoles are side-by-side as shown in Figure $7.2,(7.7)$ reduces to:

$$
\begin{aligned}
R_{2 \mathrm{Im}} & =\frac{\eta}{4 \pi}\left[2 C_{i}\left(u_{0}\right)-C_{i}\left(u_{1}\right)-C_{i}\left(u_{2}\right)\right] \quad(7.8 \mathrm{a}) \\
X_{21 m} & =-\frac{\eta}{4 \pi}\left[2 S_{i}\left(u_{0}\right)-S_{i}\left(u_{1}\right)-S_{i}\left(u_{2}\right)\right] \quad(7.8 \mathrm{~b}) \\
u_{0} & =k d \\
u_{1} & =k\left(\sqrt{d^{2}+l^{2}}+l\right) \\
u_{2} & =k\left(\sqrt{d^{2}+l^{2}}-l\right)
\end{aligned}
$$

where $C i(x)$ and $S i(x)$ are the sine and cosine integrals: 


$$
S_{i}(x)=\int_{0}^{x} \frac{\sin y}{y} d y
$$

and

$$
C_{i}(x)=-\int_{x}^{\infty} \frac{\cos y}{y} d y=\int_{\infty}^{x} \frac{\cos y}{y} d y
$$

The impedance of $(7.7)$ can be referred to the input terminal by:

$$
Z_{21 i}=Z_{21 m}\left(\frac{I_{1 m}}{I_{1 i}}\right)\left(\frac{I_{2 m}}{I_{2 i}}\right)=Z_{21 m}\left[\frac{1}{\sin \left(k l_{1} / 2\right)}\right]\left[\frac{1}{\sin \left(k l_{2} / 2\right)}\right]
$$

For identical lengths of both antennas $l_{1}=l_{2}(7.10)$ reduces to:

$$
\begin{aligned}
& R_{21 i}=\frac{R_{21 m}}{\sin ^{2}(k l / 2)} \\
& X_{21 i}=\frac{X_{21 m}}{\sin ^{2}(k l / 2)}
\end{aligned}
$$

The mutual impedance, referred to the current maximum and given by (7.7) of a side-by-side arrangement of two half-wave dipoles has been plotted [13] and is shown in Figure 7.2.

The side by side arrangement of dipoles exhibits a large mutual impedance, as compared with other arrangements (for instance, a collinear arrangement) since the antennas are placed in the direction of maximum radiation.

Also, the mutual impedance increases as the size of both sideby-side dipoles is increased, for a given wire separation. 


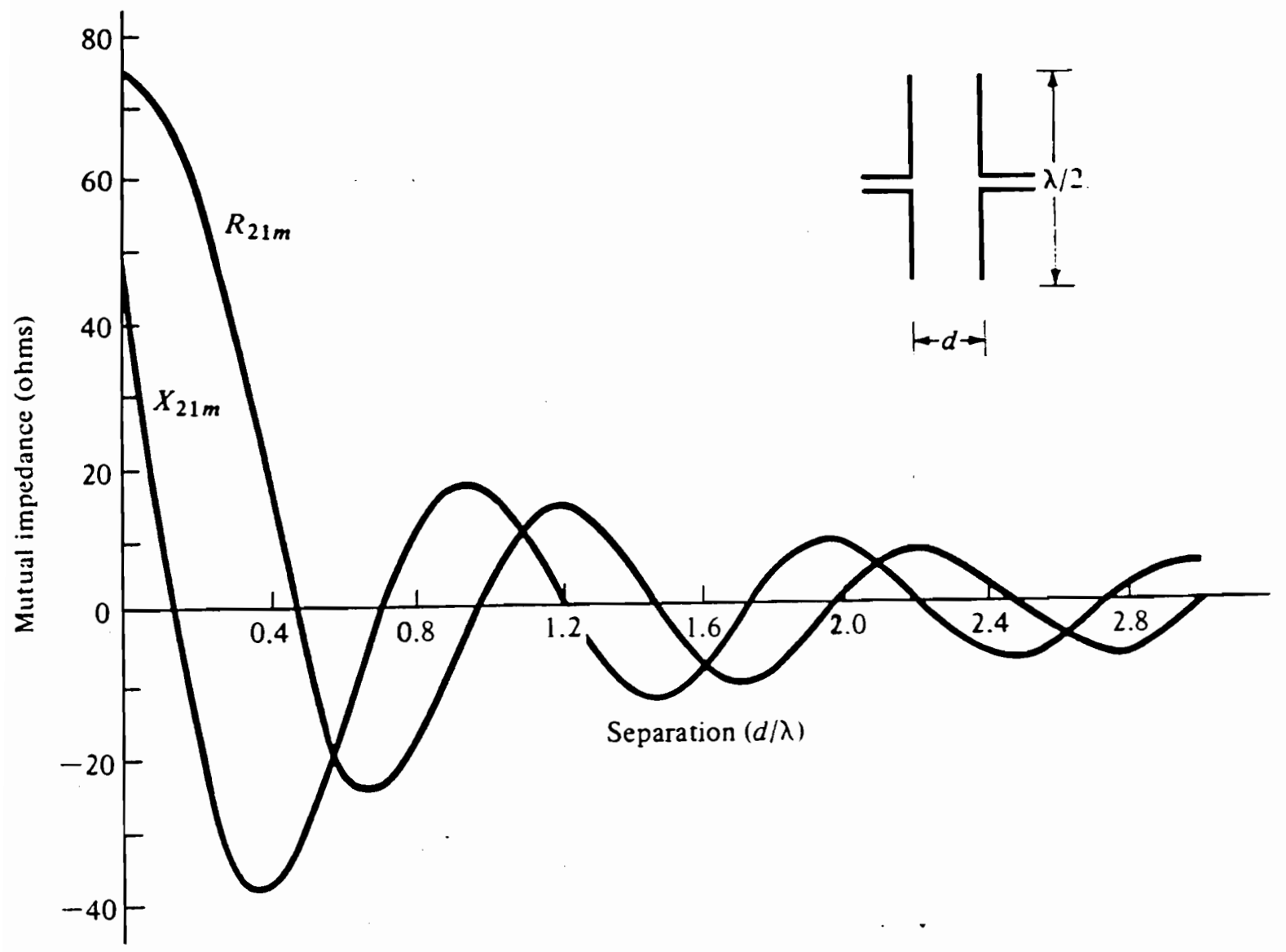

FIGURE 7.2: MUTUAL IMPEDANCE OF A SIDE-BY-SIDE ARRANGEMENT OF HALF-WAVELENGTH DIPOLES (EROM [13]) 
The low resonant resistance of a meander dipole can be explained on the basis of the mutual impedance between its parallel arms.

It is obvious, when analysing the geometry of meander antennas, that they are composed of several parallel arms, all of which contribute to the radiated field. These arms must interact between each other somehow, to vary the input impedance of the antenna.

Refering to Figure 7.3, we can model a meander dipole with $\mathrm{N}=2$, the simplest form of meander antenna, as being composed of three independent parallel wires, each having a length somewhat shorter than a half-wavelength.

In fact, for a dipole having a reduction factor of 0.6 , the length of each parallel section is:

$$
\text { or } \quad \begin{aligned}
& 1=0.6\left(\lambda_{0} / 2\right) \\
& 1=0.3 \lambda_{0}
\end{aligned}
$$

Since we can assume that the wire separation $w$ is much smaller than the wavelength, we will disregard the effect on the impedance of the small horizontal sections. These will not have a significant effect in the radiated field of the antenna, provided that $w<\lambda_{0}$

We can see from Figure 7.3 that the dipoles can be considered as having their own independent excitation.

However, from 4.2.4, we have learned that a meander dipole has a current distribution which magnitude is close to a sinusoidal distribution along the wire, and which phase remains more or less constant along the wire. We can assign to each wire an input current, that on the average, will be :

$$
\mathrm{I}_{1}>\mathrm{I}_{2}>\mathrm{I}_{3}
$$

Since $I_{1}$ and $I_{2}$ will flow in opposite directions in space, they will be considered as being $180 \mathrm{deg}$. out of phase. By contrast, since $I_{1}$ and $I_{3}$ are flowing in the same direction in space at the same time, the will be assumed as being in phase.

The input impedance of the antenna is given by (7.2): for a meander dipole $\mathbf{N}=2$ :

$$
Z(\text { input })=Z_{11}+\left(I_{2} / I_{1}\right) Z_{12}+\left(I_{3} / I_{1}\right) Z_{13}
$$



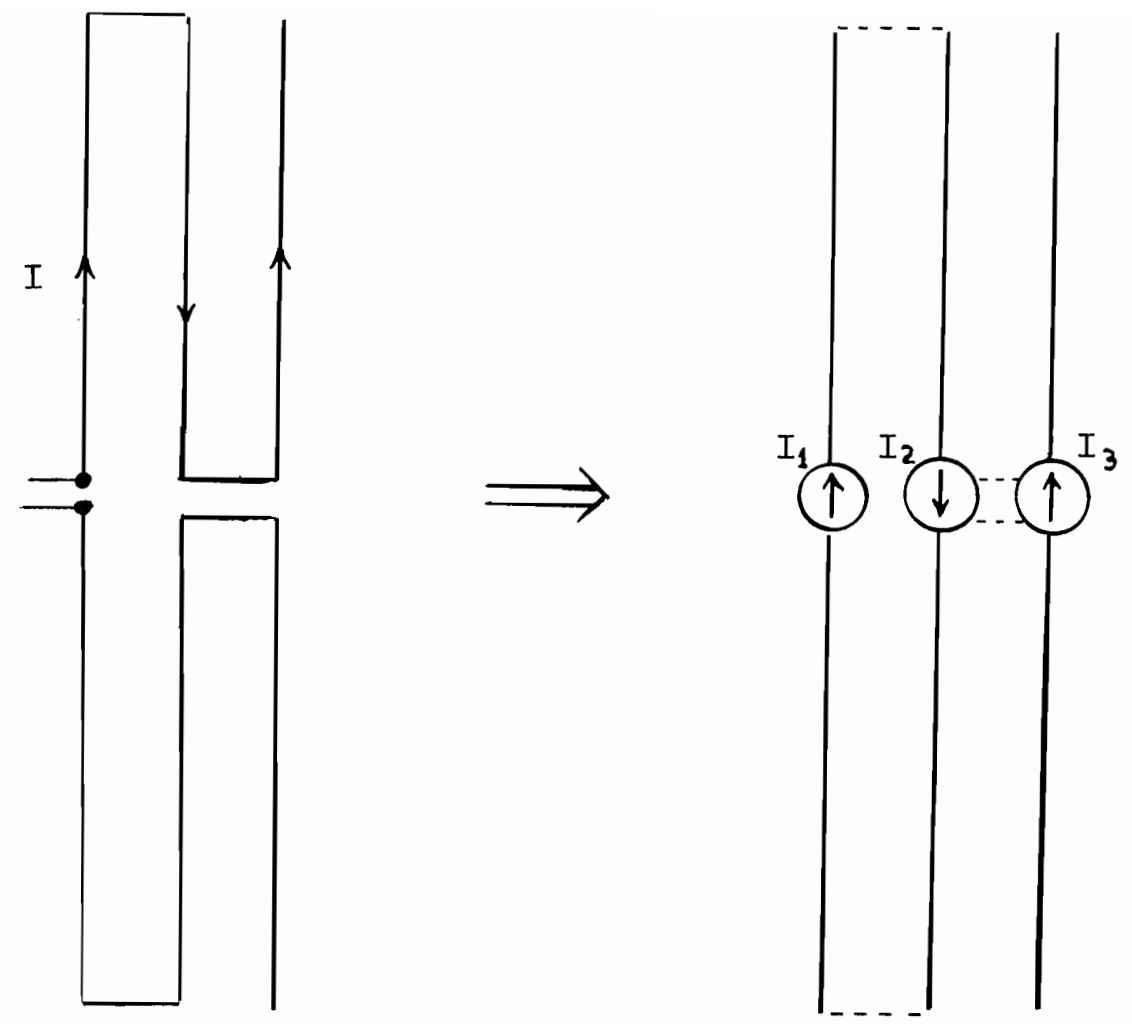

FIGURE 7.3: EQUIVALENT RE?RESENTATION OF A MEANDER DIPOLE. (The dimension of wire separation $w$ has been exagerated.) 
$Z_{11}$ is the input impedance of the first antenna with length
$I=0.3$

$\mathrm{z}_{12}$ is the mutual impedance between antenna 1 and 2

$\mathrm{Z}_{13}$ is the mutual impedance between antenna 1 and 3

Now, we know from Figure 7.2 that the mutual impedance, for dipoles placed very close to each other ( at $d=w$, for meander antennas) will be inductive and of a higher magnitude.

We have also sald that $I_{1}$ and $I_{2}$ can be considered $180 \mathrm{deg}$. out of phase and $I_{1}$ and $I_{3}$ are in phase.

Thus, (7.13) can be rewritten as:

$$
Z(\text { input })=Z_{11}-\left|I_{2} / I_{1}\right| \cdot Z_{12}+\left|I_{3} / I_{1}\right| \cdot Z_{13}
$$

where the terms $\left|I_{2} / I_{1}\right|$ and $\left|I_{3} / I_{1}\right|$ are less than 1.0 in magnitude.

The mutual impedances $z_{12}$ and $z_{13}$ being inductive, they will make the input impedance of the antenna more inductive, ( or less capacitive) This will have the effect of decreasing the resonant frequency of the antenna, which explains the sizereduction properties of meander antennas.

\subsubsection{RADIATED FIELD OF A MEANDER DIPOLE}

The radiated fields of meander antennas can be explained on the basis of the Array Theory. [4]

Refering to Flgure 7.4, we have two 1sotroplc sources separated by a distance d. If the two sources are identical in amplitude and of the same phase, and assuming they have the same polarization, the far field is given by:

$$
E=E_{1}+E_{2} e^{j \psi}
$$

where:

$$
\begin{aligned}
& E_{1}=\text { far electric field at a distance } r \text { due to source } 1 \\
& E_{2}=\text { far electric field at a distance } r \text { due to source } 2 \\
& \psi=(2 \pi d / \lambda) \cos \theta
\end{aligned}
$$

The quantity $\psi$ is the phase-angle difference between the fields of the two sources measured along the radius-vector line at the angle $\theta$ 
It has been proven that a meander antenna such as the one designed in this thesis is feasable and can be used in many practical applications.

The antenna can be used as a Folded Meander Dipole, or as a Folded Meander Monopole, as was done in the laboratory construction of chapter 6.0 .

Let us sum up the characteristics of the Meander Folded Dipole, as they compare with other conventional antennas, and with other meander geometries.

\section{RESONANT RESISTANCE:}

The resonant resistance of our prototype antenna has met the design specification stated in chapter 5.0: it is of a higher value than other meander antennas, and can be fed with a 50 ohm line, while maintaining an acceptable value of VSWR.

For, the case of a full dipole configuration, the resonant resistance can be expected to be twice the value of our experimental model, in the range of 60 to 70 ohms. Depending on the value, a 50 or 75 ohm coaxial line could be used in practice to feed this antenna.

In the case of a full Meander Folded Dipole in free space, a balun (balanced-to-balanced line transformer) would be necessary at the feed point, to connect the coaxial line. This is not a major disadvantage, since most dipole antennas that use coaxial lines, need this type of matching device.

\section{ANTENNA BANDWIDTH:}

The experimental bandwidth is higher than the one initially predicted by MININEC. We have seen that for a VSWR of 3.0 , our prototype antenna shows a bandwidth of about $4 \%$ of the resonant frequency. Although this bandwidth can be considered small when compared to those of conventional antennas, it is still quite adequate for many applications. Furthermore, the bandwidth is comparable that of meander monopoles [3] and of dipoles, as shown in chapter 5.0 .

It should be noted that a narrow bandwidth can be useful and desirable for many applications, since it adds to the selectivity of a receiving system.

\section{IMPEDANCE ADAPTABILITY:}

This is a very important feature. We have seen that the input impedance can be varied by two methods: 
In ( 7.15$)$ the reference for the phase for the two sources was taken at source 1 .

We see from (7.15), that in the case of a meander dipole the quantity $\psi$ would be very small since $d / \lambda=w / \lambda<1.0$

Therefore, the resultant far field will be equal to the sum of the contributions of each parallel arm of the meander dipole. The antenna behaves, if viewed from the far field, as one single dipole.

This explains the fact that meander antennas have a radiation pattern very similar to the one for a conventional dipole.

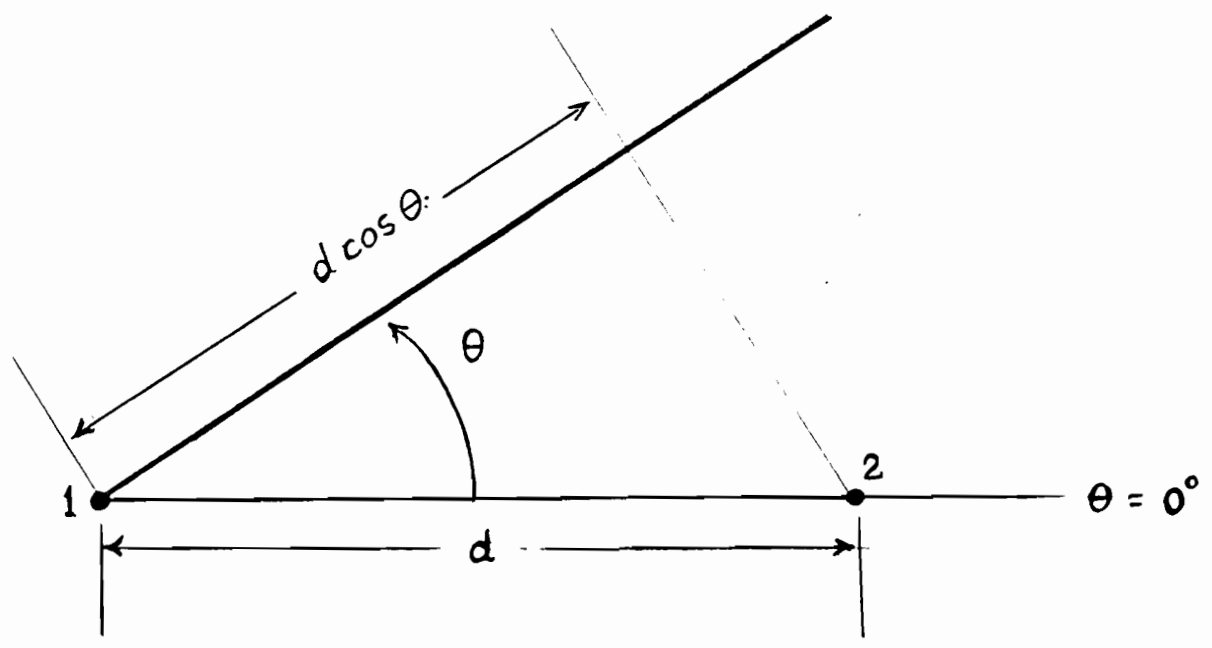

FIGURE 7.4: TWO ISOTROPIC POINT SOURCES SEPARATED BY A DIBTANCE a. 
- Changlng the wire radius, on both arms of the dipole

- Changing the wire separation w.

The wire radius method was not extensively proven in the laboratory due to practical limitations of space and equipment. However it was shown that some changes in the value of the input resistance occur as the wire-radius is changed.

The second method, is a known property of meander antennas [3] but it entails a change in bandwidth, as was seen in the experiment and in the computer simulation (refer to Chap. 5.0).

Therefore, for the case of a thin-wire Folded Meander Dipole, these methods can be used to "fine-tune" the antenna impedance, rather than to obtain drastic changes in this parameter.

\section{SIZE REDUCTION:}

The major reason for this design was of course to obtain a reduction in size over a conventional geometry.

We have obtained a size-reduction for our experimental antenna, of approximately 45\%. This is comparable, if not greater, than the size reduction obtained for meander monopoles [3]. Our major goal has been accomplished: to obtain a practical antenna that would have about half-the size of its conventional counterpart.

These characteristics of the design, are an obvious advantage over the more simpler geometries described in [3].

MININEC has been proven to be a most useful tool in the design of wire antennas. Even with complicated wire-geometries, as was the case with the Folded Meander Dipole Antenna, where the program was stretched to the limit of its capabilities, the results were reasonably close to the practlcal results. In spite of the numerical errors made by the program in calculating the antenna impedances, MININEC presented us with a good overall description of the antenna characteristics.

It $1 \mathrm{~s}$ obvious however, that a great amount of time and practice with the program is necessary, in order to entertain designs that are complex in nature. It is important to know the program's limitations and to acquire a "feel" for the accuracy of the program's results. 
The antenna designed in this thesis, was subject to nearly all the tests that MININEC could accomplish, that were useful
for our purposes.

Later, in the laboratory, the prototype antenna was also subject to as many tests were possible, within the constraints of avaliable equipment, time and physical space.

There remains to be accomplished a practical measurement of the radiation pattern of this antenna and of its radiation efficiency.

In particular the radiation efficiency, which cannot be estimated with MININEC and requires a physical measurement, is of great importance in establishing the usefulness of this antenna.

It is my hope that this design will someday be perfected and its characterization completed by future generations of betterequipped researchers. 


\section{REFERENCES}

$\vdots$

1. A.J. Jullan, J.C. Logan, J.H. Rockway, "MININEC: A Mini-Numerical Electromagnetics Code.", NOSC TD 516, september 1982.

2. J.W. Rockway, J.C. Logan, D.W.s. Tam, shing T. L1, "The Mininec System: A Mlcrocomputer Analysis of Wire Antennas". Artech House, 1985 .

3. Jal1l Rashed and Chen-To Ta1, "A new Class of wire Antennas", IEEE/AP-S Sympos1um, May 24-28, 1982.

4. John D. Kraus, "Antennas". MCGraw-Hill, 1984.

5. R.W.P. K1ng, "Tables of Antenna Characteristics." I.F.I./Plenum Corporation, 1971.

6. R. Johnson and H. Jas1k, "Antenna Englneering Handbook." McGraw-Hill 1984 .

7. G. Brown and 0. Woodward Jr., "Experimentally Determined Impedance Characteristics of Cylindrical Antennas." . IRE Proceedings, Vol. 33, 1945, p 257-262.

8. S. Uda and Y. Mushlake, "Yag1-Uda Antenna" Maruzen Co. Ltd. Tokyo 1954, p. 19

9. Y.T. Lo and S.W. Lee, "Antenna Handbook" Van Nostrand Reinhold Co., N.Y. 1988

10. A.H. RUDGE, K. Milne, A.D. Oliver, P. Knlght, "Handbook of Antenna Design." IEEE Electromagnetic Waves Series. 1984 
11. G. J. Burke and A.J. Poggio, "Numerical Electromagnetic Code (NEC) - Metbod of Moments." NosC TD 116, Jan. 1981

12. Hewlett-Packard HP-4193A vector Impedance Meter operation Manual.

13. C. Balanis, "Antenna Theory". Harper and Row Publishers. 1982 .

14. R.P. Owens, "Microstrip Antenna feeds" , Handbook of Microstrip Antennas, Vol. 2, p. 846-848. J.R. James and P.S. Hall, 1989.

15. H. Nakano, H. Tagam1, A. Yoshizawa, J. Yamauch1, "Shortening Ratios of Modifled Dipole Antennas." IEEE Transactions on Antennas and Propagation. Vol. AP-32 No.4, Apr 11 1984, pp.385-386.

16. J. Wong and H. KIng, "Helght-Reduced Zlgzag Meander Monopoles with Broadband Characteristics." IEEE Transactions on Antennas and Propagation. Vol. AP-34, May 1986, pp. 716-717. 